\title{
PREDICTING PERSISTENCE AND DESISTENCE OF RECIDIVISM IN YOUTH OFFENDERS: THE ROLE OF RISK AND PROTECTIVE FACTORS IN CRIMINAL OFFENDING
}

\author{
by \\ Krista Marie Richard \\ B.A. (Hons), St. Francis Xavier University, 1999 \\ M.A., Carleton University, 2004
}

\begin{abstract}
A dissertation submitted to
The Faculty of Graduate and Postdoctoral Affairs

in partial fulfillment of the requirements for the degree of

Doctor of Philosophy

in Psychology
\end{abstract}

Carleton University

Ottawa, Ontario

(C) August 2011, Krista Richard 
Library and Archives

Canada

Published Heritage

Branch

395 Wellington Street

Ottawa ON K1A ON4

Canada
Bibliothèque et

Archives Canada

Direction du

Patrimoine de l'édition

395 , rue Wellington

Ottawa ON K1A ON4

Canada

\section{NOTICE:}

The author has granted a nonexclusive license allowing Library and Archives Canada to reproduce, publish, archive, preserve, conserve, communicate to the public by telecommunication or on the Internet, loan, distribute and sell theses worldwide, for commercial or noncommercial purposes, in microform, paper, electronic and/or any other formats.

The author retains copyright ownership and moral rights in this thesis. Neither the thesis nor substantial extracts from it may be printed or othenwise reproduced without the author's permission.
Your file Votre référence

ISBN: 978-0-494-83221-9

Our file Notre référence

ISBN: 978-0-494-83221-9
In compliance with the Canadian Privacy Act some supporting forms may have been removed from this thesis.

While these forms may be included in the document page count, their removal does not represent any loss of content from the thesis.
L'auteur conserve la propriété du droit d'auteur et des droits moraux qui protège cette thèse. $\mathrm{Ni}$ la thèse ni des extraits substantiels de celle-ci ne doivent être imprimés ou autrement reproduits sans son autorisation.

\section{AVIS:}

L'auteur a accordé une licence non exclusive permettant à la Bibliothèque et Archives Canada de reproduire, publier, archiver, sauvegarder, conserver, transmettre au public par télécommunication ou par l'Internet, prêter, distribuer et vendre des thèses partout dans le monde, à des fins commerciales ou autres, sur support microforme, papier, électronique et/ou autres formats.

Conformément à la loi canadienne sur la protection de la vie privée, quelques formulaires secondaires ont été enlevés de cette thèse.

Bien que ces formulaires aient inclus dans la pagination, il n'y aura aucun contenu manquant. 
[ii] 


\begin{abstract}
Researchers have found a strong association between adult psychopathy and serious repetitive crime, violence, and mixed treatment outcome (Hare, Clark, Grann, \& Thornton, 2000; Hemphill, Hare, \& Wong, 1998; Ogloff, Wong, \& Greenwood, 1990; Olver, Stockdale, \& Wormith, 2009; Rice, Harris, \& Cormier, 1992), which has resulted in increased attention of applying the downward extension of identifying psychopathic traits in youth. Further, over the past decade researchers have developed assessment measures to determine the level of risk and dangerousness among adolescents (Borum, Bartel, \& Forth, 2006). The objective of this dissertation was three-fold: to distinguish if certain factors would differ between youth who persisted in their offending and those who desisted; to determine the predictiveness of two assessment inventories and the number of conduct disorder symptoms on future offending; and finally, to discern if protective factors, alone or in combination with risk factors were related to either persistence or desistence of offending. This study examined 235 male youth offenders who were either serving or were remanded to custody in a youth detention facility. The findings suggest that psychopathy is less related to the long term predictive ability of future criminal offending than the examination of the risk of violence in youth, as examined by the Structured Assessment of Violence Risk in Youth (SAVRY, Borum et a., 2006). The finding that the Psychopathy Checklist: Youth Version (PCL:YV, Forth, Kosson \& Hare, 2003) is not as predictive of future offending as the SAVRY risk ratings is consistent with previous studies. Further, the AUCs for the SAVRY indicate that this instrument has an ability to predict violent offending. Collectively, the results contribute to the predictively validity research for the PCL:YV and the SAVRY for short and long-term periods. The results further demonstrate that
\end{abstract}


future applications of these scales should differ based on the specific outcome purpose. In other words, the PCL:YV and the SAVRY should not be used interchangeably as an assessment of risk, as the findings of the current study suggest that the SAVRY may be a more appropriate tool for such assessment amongst male youth. 


\section{Acknowledgements}

To my advisor, Adelle, you truly are an inspiration to students. You are a kind and patient professor who cares about your students above and beyond what is called for and I truly am so grateful that I got to learn from you, be guided by you, and to fulfill my dream of pursuing this degree with your support. I will always be grateful of everything you have done for me and I am indebted to you for all your support. Thank you to my committee members - Dr. Craig Bennell, Dr. Ralph Serin and Dr. Kevin Nunes. You have all provided generous support and encouragement throughout my degree and I will never forget your help. I would also like to extend my appreciation to Drs. Mary Ann Campbell and Diana Majury for your thoughtful comments as external examiners. It was a pleasure meeting you both.

I would like to thank my dear friend, Jillian Flight, for always being there for me. You are such an inspiration and I feel truly blessed that you are in my life. You have listened to my many rants about my academic endeavors and you have always been there to listen and provide advice for these paths. I am always humbled by your expertise and knowledge and your unwavering support. You have taught me to not sweat the small stuff and to know how to laugh in the face of adversity. I truly value the friendship we share.

To my family, you have always been there for me whenever I needed you. I am the person I am today because of your support, love and guidance. Thank you Mom and Dad for knowing that my limits are only my own and realizing the faith you have in me has only made me a much better person. Thank you Jennifer for teaching me how to laugh and to know I have a big sister who is always available to listen to my every concern. I love you. 
This dissertation is dedicated to my best friend and husband, Stuart and our beautiful daughter Addison. You both are my inspiration and my reason for happiness. Stuart - thank you for giving me the support to complete this dissertation. You have had many nights of me "doing my school work" and having to take on responsibilities on your own. I am so grateful and appreciate all that you have done so that I can fulfill this dream. You are my rock and I love you with all my heart. Addison - my precious girl - you make me smile at every moment. You are such a wonderful daughter and I am so lucky to be your Mommy. I cannot wait to see what the future brings for you, Daddy, and me. 


\section{Table of Contents}

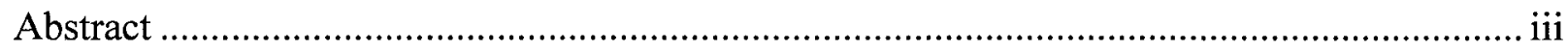

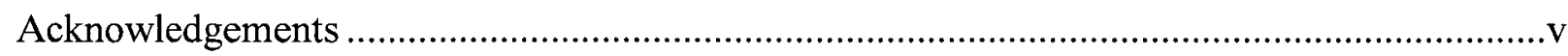

Table of Contents ...................................................................................................... vii

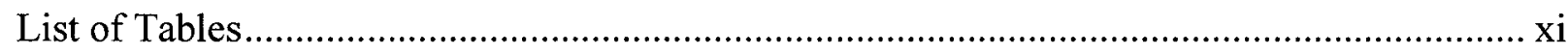

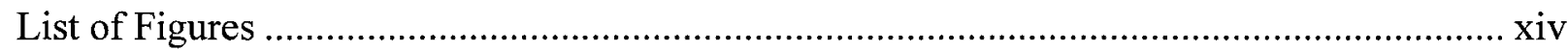

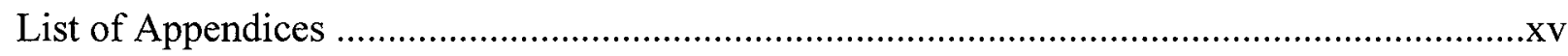

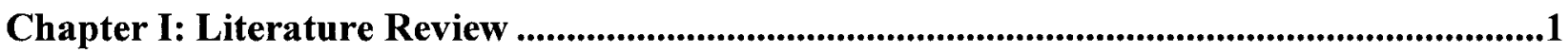

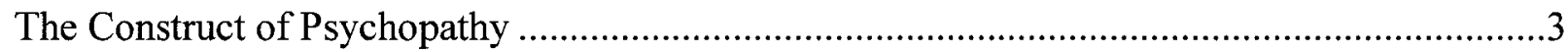

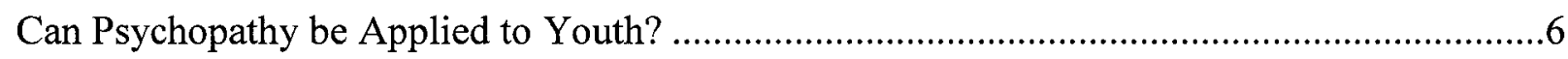

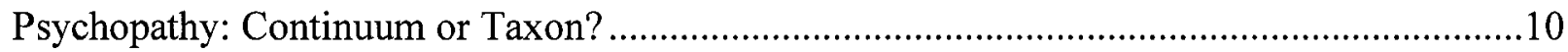

Challenges with Measuring Psychopathic Traits in Youth ................................................12

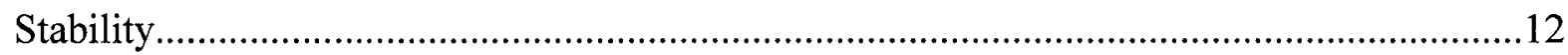

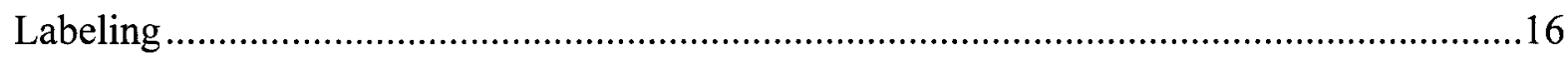

Developmental Perspective ................................................................................. 17

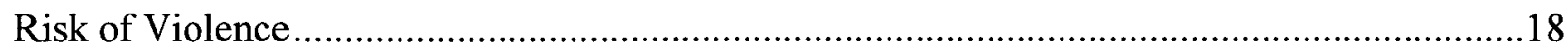

Criminal Behaviour - Theory and Developmental Perspective ..........................................21

Desistence from Offending - Role of Protective Factors ................................................24

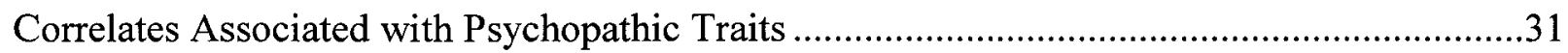

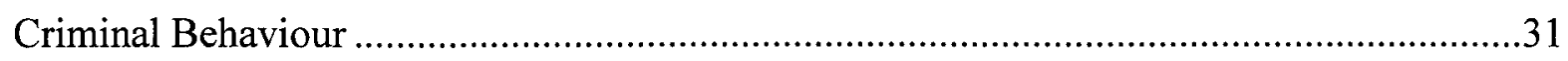

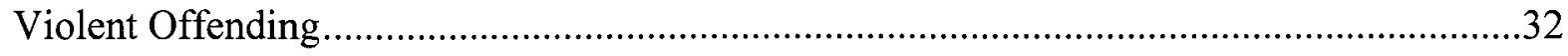




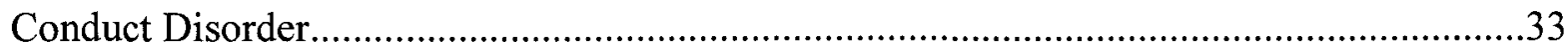

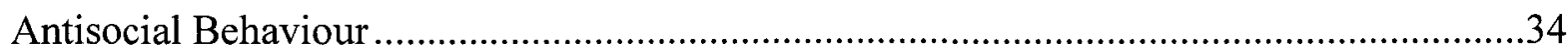

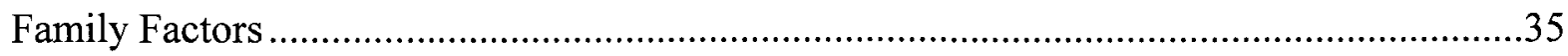

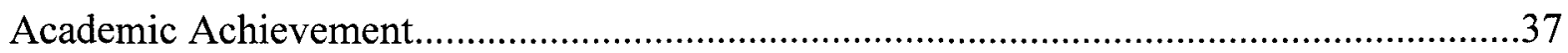

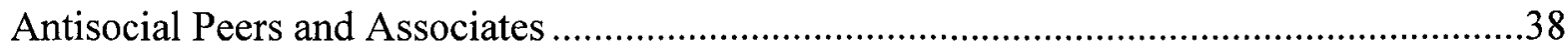

Predictive Validity of Risk Assessments in Youth - PCL:YV and SAVRY .............................40

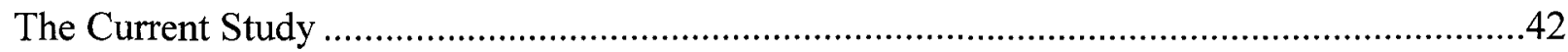

Chapter II: Method.......................................................................................................................................45

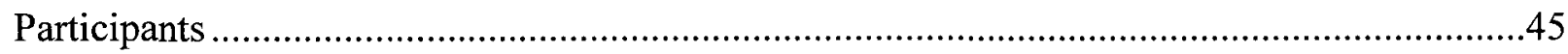

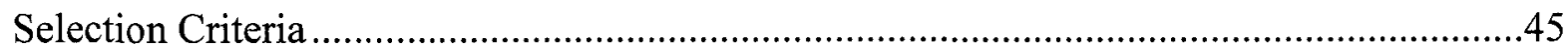

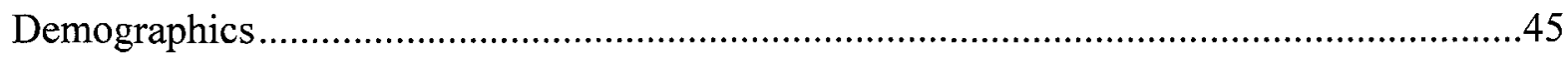

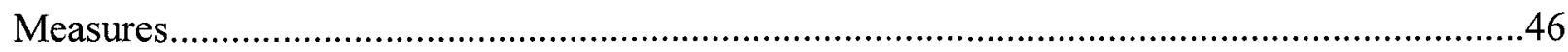

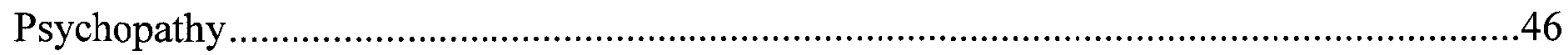

Risk of Violence

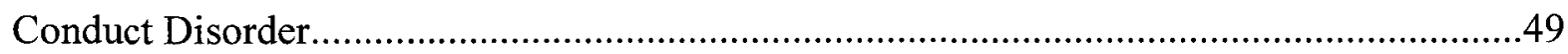

Canadian Police Information Centre (CPIC) Records........................................................49

Factors Associated with Psychopathy and Risk of Violence.......................................................50

Procedure

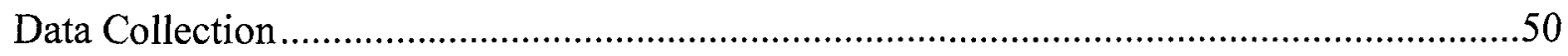

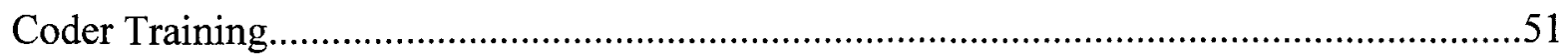

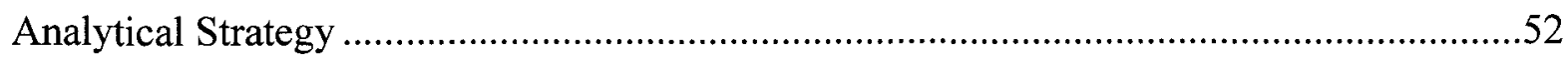


Chapter III: Results ........................................................................................................................55

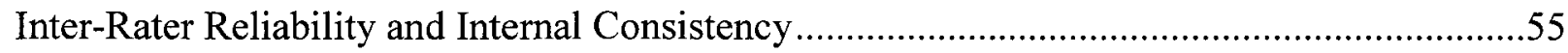

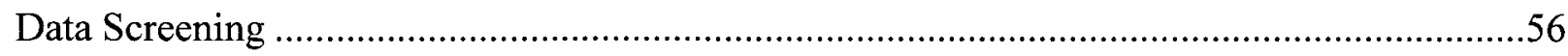

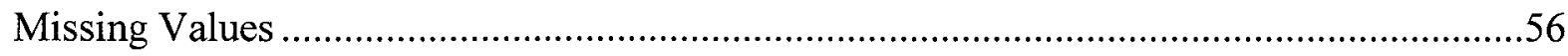

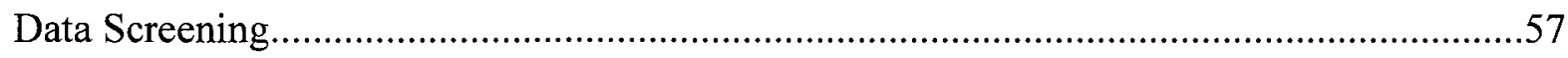

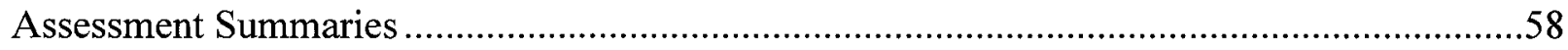

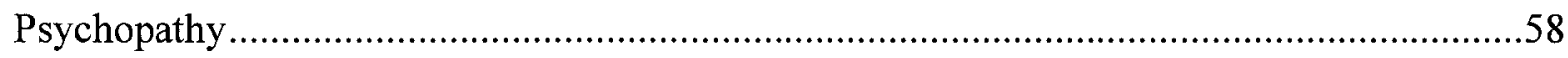

SAVRY

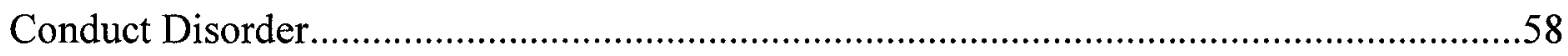

Prior Offence Characteristics ...............................................................................................59

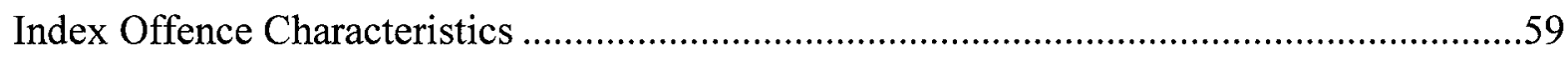

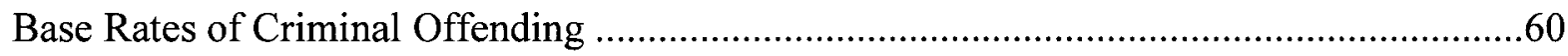

Comparisons between Persisters and Desisters - Objective One ……………………..............63

Role of Risk Factors in the Prediction of Criminal Offending - Objective Two........................69

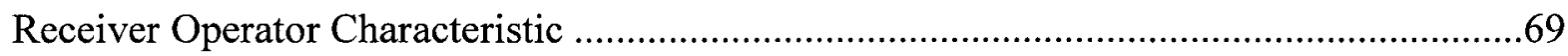

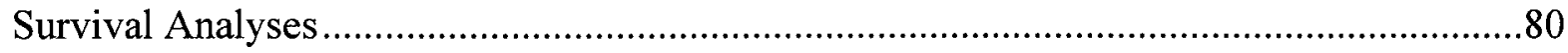

Role of Protective Factors in Criminal Desistence - Objective Three.....................................102

Chapter IV: Discsussion ..............................................................................................................................117

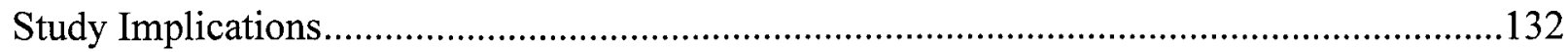

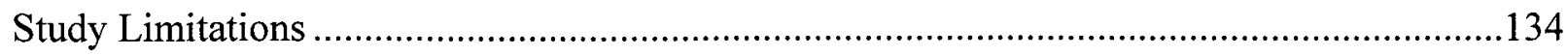

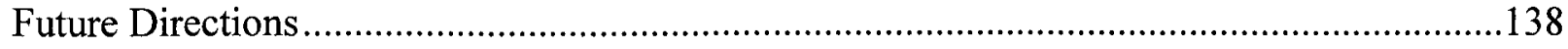


Persistence and Desistence of Offending $\mathrm{x}$

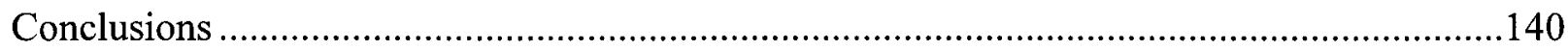

Chapter V: References ......................................................................................................................142

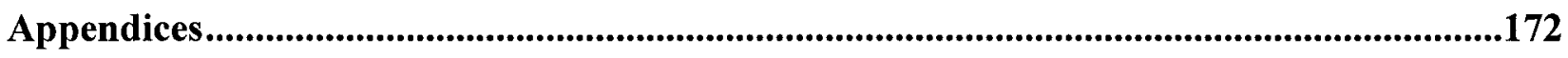




\section{List of Tables}

Table 1: PCL:YV Factor Structure and Items for 3-Factor and 4-Factor Models 9

Table 2: Descriptive Statistics of Total Sample .46

Table 3: Internal Consistency and Inter-Rater Reliablity for PCL:YV, SAVRY and Conduct

Disorder. 56

Table 4: Descriptive Summary for PCL:YV, SAVRY and CD Symptoms 57

Table 5: Correlations between PCL:YV, SAVRY and CD Symptoms .59

Table 6: Base Rates of Offending .61

Table 7: Youth who either Persisted or Desisted from Non-Violent Offending: A Comparison of the PCL:YV, SAVRY and CD Scores 64

Table 8: Youth who either Persisted or Desisted from Violent Offending: A Comparison of the PCL:YV, SAVRY and CD Scores 65

Table 9: Youth who either Persisted or Desisted from Non-Violent Offending: A Comparison of

Prior and Index Offences .66

Table 10: Youth who either Persisted or Desisted from Violent Offending: A Comparison of

Prior and Index Offences .67

Table 11: Youth who either Persisted or Desisted from Non-Violent Offending: A Comparison of Clinical, Social, Familial and Academic Factors .68

Table 12: Youth who either Persisted or Desisted from Violent Offending: A Comparison of

Clinical, Social, Familial and Academic Factors .69

Table 13: ROC Results for General Recidivism for Entire Follow-up Period ...............................70

Table 14: ROC Results for General Recidivism for One Year Follow-up ....................................71 
Table 15: ROC Results for General Recidivism for Five Year Follow-up......................................72

Table 16: ROC Results for General Recidivism for Ten Year Follow-up .....................................74

Table 17: ROC Results for Non-Violent Recidivism for One Year Follow-up .............................75

Table 18: ROC Results for Non-Violent Recidivism for Five Year Follow-up ..............................76

Table 19: ROC Results for Non-Violent Recidivism for Ten Year Follow-up ...............................77

Table 20: ROC Results for Violent Recidivism for One Year Follow-up.....................................78

Table 21: ROC Results for Violent Recidivism for Five Year Follow-up .....................................79

Table 22: ROC Results for Violent Recidivism for Ten Year Follow-up .....................................80

Table 23: Cox Regression - One Year Follow-up......................................................................83

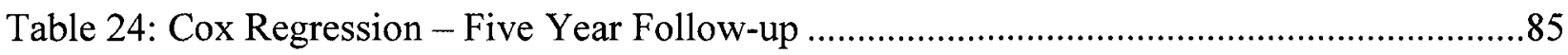

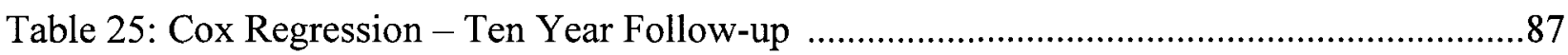

Table 26: Mean Survival Times for PCL:YV and SAVRY Groups.............................................88

Table 27: Base Rates of Re-Offending for Non-Violent and Violent Offences Based on the

Number of Protective Factors Present 102

Table 28: Youth who either Persisted or Desisted from Non-Violent Offending: A Comparison

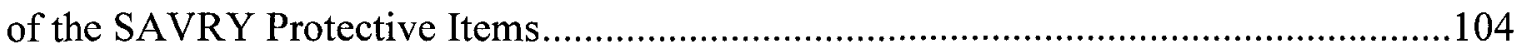

Table 29: Youth who either Persisted or Desisted from Violent Offending: A Comparison of the

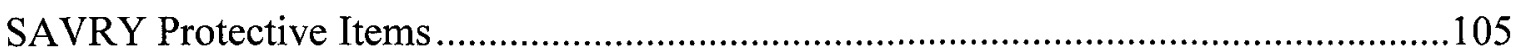

Table 30: T-tests Comparing the Number of Non-Violent and Violent Offences per Each

SAVRY Protective Item

Table 31: Univariate Logistic Regression for SAVRY Protective Items Predicting Any Non-

Violent Recidivism 109 
Table 32: Mulivariate Logistic Regression for SAVRY Protective Items Predicting Any Non-

Violent Recidivism

Table 33: Univariate Logistic Regression for SAVRY Protective Items Predicting Any Violent

Recidivism

Table 34: Mulivariate Logistic Regression for SAVRY Protective Items Predicting Any Violent

Recidivism

Table 35: Incremental Validity of the PCL:YV over the SAVRY Protective Rating for the

Prediction of Non-Violent and Violent Offending

Table 36: Incremental Validity of the SAVRY Risk Rating over the SAVRY Protective Rating

for the Prediction of Non-Violent and Violent Offending

Table 37: Demographic and Outcome Measure Comparison Across the Three Samples.

Table 38: Ethnic Comparisons Across the Three Samples

Table 39: Prior and Index Offence Rates Across the Three Samples

Table 40: Comparisons Across Samples on Predictive Accuracy of Risk Measures for General

Recidivism .192

Table 41: Comparisons Across Samples on Predictive Accuracy of Risk Measures for Non-

Violent Recidivism 193

Table 40: Comparisons Across Samples on Predictive Accuracy of Risk Measures for Violent

Recidivism 


\section{List of Figures}

Figure 1: Base Rates of Non-Violent and Violent Offending by PCL :YV Groups......................61

Figure 2: Base Rates of Non-Violent and Violent Offending by SAVRYGroups .......................62

Figure 3: Kaplan-Meier Graph - One Year Non-Violent Recidivism for PCL :YV Groups ........90

Figure 4: Kaplan-Meier Graph - Five Year Non-Violent Recidivism for PCL :YV Groups........91

Figure 5: Kaplan-Meier Graph - Ten Year Non-Violent Recidivism for PCL :YV Groups ........92

Figure 6: Kaplan-Meier Graph - One Year Non-Violent Recidivism for SAVRY Groups...........93

Figure 7: Kaplan-Meier Graph - Five Year Non-Violent Recidivism for SAVRY Groups ..........94

Figure 8: Kaplan-Meier Graph - Ten Year Non-Violent Recidivism for SAVRY Groups ...........95

Figure 9: Kaplan-Meier Graph - One Year Violent Recidivism for PCL :YV Groups .................96

Figure 10: Kaplan-Meier Graph - Five Year Violent Recidivism for PCL :YV Groups..............97

Figure 11 Kaplan-Meier Graph - Ten Year Violent Recidivism for PCL :YV Groups.................98

Figure 12: Kaplan-Meier Graph - One Year Violent Recidivism for SAVRY Groups................99

Figure 13: Kaplan-Meier Graph - Five Year Violent Recidivism for SAVRY Groups ..............100

Figure 14: Kaplan-Meier Graph - Ten Year Violent Recidivism for SAVRY Groups ...............101 


\section{List of Appendices}

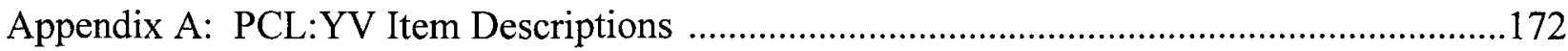

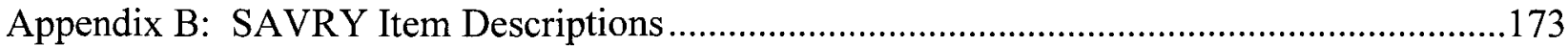

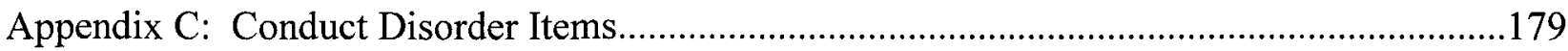

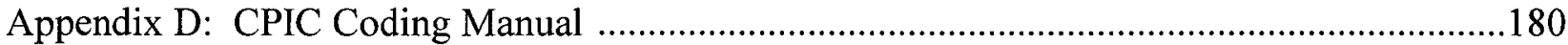

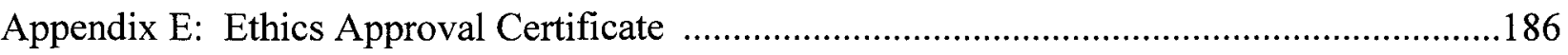

Appendix F: Comparisons Across Three Samples ……......................................................187 


\section{Chapter I: Literature Review}

Given how pervasive criminal offending is in our society, it is not surprising why many researchers have devoted their careers to try and understand this complex behaviour. Criminal behaviour has fascinated us as a society as one only needs to look at the plethora of television shows that sensationalize criminals to know that we have a need to understand why people engage in this type of antisocial behaviour. Some theorists have been interested in the personality traits associated with criminal offending and how these traits develop and change over the course of one's lifetime (Andrews \& Bonta, 2010; Lynam, 1996; Moffitt, 1993). One such personality disorder that has captured the attention of many researchers over the past few decades is psychopathy. This personality is characterized by disturbances in interpersonal and affective functioning and impulsive behavioural and antisocial tendencies (Hare, 2003) and has been linked to violence and criminal recidivism (Leistico, Salekin, DeCoster, \& Rogers, 2008).

Researchers have found a strong association between adult psychopathy and serious repetitive crime, violence, and mixed treatment outcome (Hare, Clark, Grann, \& Thornton, 2000; Hemphill, Hare, \& Wong, 1998; Ogloff, Wong, \& Greenwood, 1990; Olver, Stockdale, \& Wormith, 2009; Rice, Harris, \& Cormier, 1992), which has resulted in increased attention of applying the downward extension of identifying psychopathic traits in youth. However, concerns have been raised regarding the challenges and appropriateness of assessing these traits among adolescents. Edens, Skeem, Cruise, and Cauffman (2001) argue that adolescence is a period of enormous developmental change and that some juveniles engage in normative behaviours that include considerable delinquent behaviour. Edens and colleagues (2001) postulate that "it seems problematic to ascribe presumably immutable psychopathic 
characteristics to adolescents whose socio-emotional abilities are not fully developed" (p. 59) and that assessment tools designed to measure psychopathy in adolescence may tap constructirrelevant variance (Messick, 1995) that is associated with normative and temporary characteristics of adolescence rather than the deviant and stable personality features (Edens et al., 2001).

Further to the expansion of applying the psychopathic construct with youth, over the past decade researchers have developed assessment measures to determine the level of risk and dangerousness among adolescents (Borum et al., 2006). These measures have been used in sentencing and release, psychiatric emergency services, civil psychiatric hospitals, and outpatient clinics (Borum, 2000) to inform from a risk assessment/management model (Borum et al., 2006). This model stresses that risk of violence or dangerousness should be seen as contextual and is highly dependent on situations and circumstances, is dynamic and continuous (National Research Council, Committee on Risk Perception and Communications, Commission on Behavioral and Social Sciences and Education, Commission on Physical Sciences, Mathematics, and Resources, 1989). In other words "the task is to determine the nature and degree of risk a given individual may pose for certain kinds of behaviours in light of anticipated conditions and contexts" (Borum et al., 2006, p. 2).

Given that both the construct of youth psychopathy and measures designed to assess the risk of violence and dangerousness in youth have been studied as a means of identifying those at subsequent risk of serious, repetitive criminal behaviour, this dissertation was designed to examine three main objectives: (1) to examine if those who persist ${ }^{1}$ in offending differ from

\footnotetext{
${ }^{1}$ The term persister was used here to refer to youth who went on to re-offend post index, whereas the term desister was used to describe youth who did not recidivate post index. Although the term recidivist/non-recidivist is sometimes used in the literature to describe non-trajectory type studies, the terms persister and desister were chosen to highlight the seriousness of identifying youth who potentially are at heightened risk of repeated criminality.
} 
those who desist in offending on certain background, family and social factors. Several researchers have found correlates of criminal behaviour but few have studied factors that may relate to the desistance of offending; (2) to determine whether certain factors can predict the likelihood and imminence of offending, both short and long-term for non-violent, violent and sexual offences. More specifically, can risk assessment measures administered when participants were incarcerated for youth offences, predict outcomes at 1 year, 5 year and 10 year post release? and lastly, (3) can risk instruments, in conjunction with protective factors distinguish between persisters and desisters of offending?

\section{The Construct of Psychopathy}

The psychopathic personality has captivated clinicians for decades (Hervé, 2007).

Although the nomenclature used to refer to psychopathy has changed over the years - manie sans delire (mania without delirium, Rotenburg \& Diamond, 1971) moral insanity (Herpertz \& Sass, 2000), and sociopathy (Pennington, 1966) to name a few - the construct as it is currently defined was most notably influenced by the work of Hervey Cleckley. Cleckley's (1976) Mask of Sanity provided specific symptoms that were based upon clinical case examples and has shown to have influenced how the construct would later be defined by others (Hare, 1991). Cleckley (1976) defined core characteristics associated with the psychopathic personality. He described it as personality plagued by a constellation of positive and negative traits such as superficial charm, tendency to manipulate others, insincerity, shallow affect, pervasive antisocial behaviours, and other behavioural, interpersonal and affective features.

Influenced by the work of Cleckley, Robert Hare and his colleagues began to operationally define the personality and behavioural indices associated with psychopathy into a clinical rating scale. The Hare Psychopathy Checklist (PCL; Hare, 1980) and the later version, 
The Hare Psychopathy Checklist-Revised (PCL-R; Hare, 1991, 2003) were developed to provide an indication of the degree to which an individual possesses traits associated with a prototypical profile of a psychopath (Hervé, 2007). The scales were designed to measure items on a threepoint scale: 0 (definitely does not apply), 1 (somewhat applies) and 2 (definitely applies), thus providing an overall composite to yield one as either high or low on the psychopathic scale. Scores can range from 0 to 40 , with a score of 30 or above used to diagnose psychopathy (Hare, 2003). Individuals who score less than 21 on this scale are referred to as non-psychopaths. The reliability and validity of this assessment tool has been strongly demonstrated among numerous populations, including criminal offenders (Hare, 1996; Hare, Forth, \& Stachan, 1992), sex offenders (Olver \& Wong, 2009), civil psychiatric patients (Douglas, Ogloff, Nicholls, \& Grant, 1999; McDermott et al., 2000; Skeem \& Mulvey, 2001), forensic psychiatric patients (Chakhssi, de Ruiter \& Bernstein, 2010), female offenders (Coid et al., 2009), and African American offenders (Walsh \& Kosson, 2007).

Early factor analyses conducted on the instrument yielded two distinct, albeit correlated, dimensions. Hare (1991) classified these as Factor 1 (interpersonal/affective) and Factor 2 (lifestyle). Factor 1 includes such traits as glibness and superficial charm, grandiose sense of self-worth, pathological lying, manipulativeness, lack of remorse, shallow affect, callousness and lack of empathy, and failure to accept responsibility. The second factor identifies traits more closely associated with a chronically, unstable antisocial lifestyle. The traits included in this factor are: need for stimulation and proneness to boredom, parasitic lifestyle, poor behavioural controls, early behavioural problems, lack of realistic long-term goals, impulsivity, irresponsibility, and juvenile delinquency. 
Cooke and Michie (2001) hypothesized that a three factor hierarchical solution better describes the features of psychopathy. Through the use of confirmatory factor analysis, Cooke and Michie (2001) concluded that the two-factor model did not adequately explain the factors of this disorder. Cooke and Michie (2001) examined the PCL-R on the basis of three important factors described by Cleckley (1976) and Hare (1991) as the core features of this disorder: affective, interpersonal, and behavioural qualities. By eliminating seven items of the PCL-R this new factor structure places less emphasis on specific antisocial behaviours and rather shifts more focus on personality traits (Cooke \& Michie, 2001). This factor structure deemphasizes criminal behaviour more so than does the two-factor structure described by Hare et al. (1990) (Cooke \& Michie, 2001; Cooke, Michie, \& Skeem, 2007). Further exploration into the factor structure of the PCL-R has recently discovered that a 4-factor model of psychopathy may provide the most comprehensive explanation into the manifestation of this disorder (Babiak, Neumann, \& Hare, 2010; Hare \& Neumann, 2008, 2010). This model incorporates the same factors as the 3 -factor model identified by Cooke and Michie (2001), however it adds a fourth dimension that consists of the five of the seven items that Cooke and Michie (2001) excluded from their model. This fourth dimension captures early onset antisocial behaviour and of problematic behaviours. Support for the 4-factor model is supported from research based on clinical, community and forensic populations (e.g., Babiak et al., 2010; Hare \& Neumann, 2008, 2010).

In summary, the PCL-R is extensively used in both research and clinical practice and has been validated and shown to be a reliable measure of the features believed to be fundamental to the construct of psychopathy. 


\section{Can Psychopathy be Applied to Youth?}

Exploring whether psychopathic traits emerge in adulthood or is best explained as a developmental process that begins in childhood has received increased attention over the past few years (Farrington, 2005; Forth \& Book, 2007, 2010; Forth \& Burke, 1998; Forth \& Mailloux, 2000; Frick, 2000; Frick, O’Brien, Wootton, \& McBurnett, 1994; Frick \& Hare, 2001; Lynam, 1996). Some researchers argue that psychopathy does not simply reveal itself in adulthood (Forth \& Burke, 1998) and that psychopathic traits in youth are manifested at an early age (Forth \& Book, 2007, 2010; Forth \& Mailloux, 2000; Frick, 1998; Johnstone \& Cooke, 2004; Lynam, 2002; Saltaris, 2002). However, research findings are beginning to characterize the importance of differentiating amongst youth with other early conduct disorders (Gretton, Hare, \& Catchpole, 2004).

Studies have shown that adolescence is marked by an increase in the number of antisocial behaviours (Farrington, 1983; Wolfgang, Thornberry, \& Figlio, 1987), although not all youth who commit acts of aggression are found to continue this aggression into adulthood (Piquero, Farrington, Nagin, \& Moffitt, 2010). It is because of this finding that researchers have studied correlates to antisocial behaviour as a means of determining if such a person suffers from a mental illness, a psychological disorder, emotional disturbance, or learning or mental disability. Through assessments, researchers are able to determine if certain youths are at risk for serious chronic offending and violence. It is through identifying those with these risk factors that intervention and management strategies can be established to reduce this trajectory (Gretton et al., 2004)

The Hare PCL:YV (Forth et al., 2003) was developed to assess features of psychopathy in an adolescent population. This tool was modified from its parent tool, the PCL-R (Hare, 2003) 
to reflect the age appropriateness of the target population. The item descriptions of the PCL:YV take into account the behavioural qualities of adolescent life and the restrictions this age group experiences (Forth et al., 2003). The PCL:YV retains all 20 original PCL-R items, however specific modifications were made to make it more appropriate for adolescents. Further, instructions were revised to emphasize the nature of normal adolescent behaviour and its variability over time. More focus was given to enduring features of youth that are present across settings and time (Forth et al., 2003). Akin to the PCL-R, multiple collateral sources are encouraged to ensure that the frequency, intensity, and duration of each item are assessed accurately (Forth et al., 2003). Similarly to the PCL-R, each item is scored as either a 2 (definitely applies), 1 (somewhat applies) or 0 (definitely does not apply). The items are summed to determine an overall score with a range from 0 to 40 .

In the development of the PCL:YV, Forth et al. (2003) utilized a downward extension of the concept as it applies to adults. This is based on the assumption that psychopathic traits in childhood and adolescence would closely resemble psychopathic traits in adulthood (Lynam, 1998). However, some argue that differences in age, experience, cognitive and emotional development, and other developmental factors would influence the symptoms and thus present differently in childhood (Frick, 1998; Ollendick \& Vasey, 1999; Silverthorn \& Frick, 1999). Lynam (1998) found that children who exhibited symptoms of hyperactivity, impulsivity, and attention problems (HIA) and who also showed conduct problems (CP) most closely resembled psychopathic adults. He further noted that boys who exhibited all these symptoms were more psychopathic than those who exhibited HIA or CP alone. Lynam (1996) argued that children who exhibited HIA and CP were "affected with a virulent strain of conduct disorder best 
described as fledgling psychopathy" (p. 209). However, his assertion into the manifestation of psychopathic tendencies in children only takes into account behavioural aspects of this disorder.

Utilizing an exploratory and confirmatory factor analysis, Forth et al. (2003) examined 505 incarcerated juvenile male offenders and a pooled sample of 1631 that comprised of both male and female offenders to examine the factor structure of the PCL:YV. These analyses revealed both a 3-Factor and a 4-Factor model that encompasses similar dimensions as outlined by the PCL-R in adult populations. The 3-Factor model incorporates affective, behavioural and interpersonal dimensions, whereas the 4-Factor model adds a fourth component, antisocial behaviour, and is believed to be a better fitting model in explaining the factors that distinguish juvenile psychopathic traits (Forth et al., 2003; see Table 1 for factor items). 
Table 1

PCL:YV Factor Structure and Items for 3-Factor and 4-Factor Models

Three Factor Model

Factor 1: Interpersonal

1. Impression Management

2. Grandiose Sense of Self-Worth

3. Pathological Lying

4. Manipulation for Personal Gain

Factor 2: Affective

1. Lack of Remorse

2. Shallow Affect

3. Callous/Lack of Empathy

4. Fails to Accept Responsibility

Factor 3: Behavioural

1. Stimulation Seeking

2. Impulsivity

3. Irresponsibility

4. Parasitic Orientation

5. Lacks Goals
Four Factor Model

Factor 1: Interpersonal

1. Impression Management

2. Grandiose Sense of Self-Worth

3. Pathological Lying

4. Manipulation for Personal Gain

Factor 2: Affective

1. Lack of Remorse

2. Shallow Affect

3. Callous/Lack of Empathy

4. Fails to Accept Responsibility

Factor 3: Behavioural

1. Stimulation Seeking

2. Impulsivity

3. Irresponsibility

4. Parasitic Orientation

5. Lacks Goals

Factor 4: Antisocial

1. Poor Anger Control

2. Early Behavioural Problems

3. Serious Criminal Behaviour

4. Violations of Conditional Release

5. Criminal Versatility

Note: PCL:YV = Psychopathy Checklist: Youth Version

Neumann, Kosson, Forth, and Hare (2006) examined the factor structure of the PCL:YV in a male adolescent sample to determine if the factor structure hypothesized to fit with adult samples would be observed with an incarcerated youth sample. The researchers tested both the 3factor model developed by Cooke and Michie (2001) and the 4-factor model developed by Hare (2003) and found that both models were associated with generally good fit. However, the authors 
concluded that given both models were found to have similar fits, the 4-factor model should be the preferred model for explaining the structure of PCL-based psychopathy. Given that the antisocial factor has been found to be related to later externalizing pathology (Rutter, Giller \& Hagell, 1998) and psychopathy (Lynam, 1996, 1998) and that it is moderately correlated with the other factors, Neumann et al. (2006) stated that antisocial behaviours represent a component of the psychopathic construct that should not be omitted.

Jones, Cauffman, Miller, and Mulvey (2006) explored the factor structure of the PCL:YV to discern if differences across gender and race/ethnicity would emerge. Their study provided additional support for both the 3-factor (Cooke \& Michie, 2001) and the 4-factor (Hare, 2003). Although the authors state that the 3-factor model is more parsimonious than the 4-factor model, it may undermine the integrity of the psychopathic construct. In addition, this study found that the factor structure did not vary across different racial/ethnic groups (Caucasian, African American or Latino) or across gender (Jones et al., 2006).

Taken together, these studies indicate that the PCL:YV appears to be similar to the PCL$\mathrm{R}$ in that it is both valid and reliable amongst youth populations. Given that researchers have demonstrated that both the 3-factor and 4-factor models appear to have similar fits (Cauffman, Kimonis, Dmitrieva, \& Monahan, 2009; Forth et al., 2003; Jones et al., 2006; Neumann et al., 2006; Salekin, Brannen, Zalot, Leistico \& Neumann, 2006), the 4-factor model appears to be the most comprehensive in explaining all the features associated with psychopathy.

\section{Psychopathy: Continuum or Taxon?}

Whether psychopathy is a function of a diagnostic category or continuum has led to several empirically based studies to be conducted regarding this disorder (Blackburn \& Coid, 1998; Harris, Rice, \& Quinsey, 1994). Harris and colleagues (1994) proposed that psychopathy 
should be regarded as a taxonic disorder. Using PCL-R data on a group of maximum security psychiatric inmates, Harris and colleagues utilized curve fitting procedures to reveal two distinct normal distributions. Lilenfeld (1998) however, noted that Harris et al. (1994) did not find evidence of a taxon with Factor 1 (interpersonal/affective), but only in Factor 2 (antisocial lifestyle/behaviour). Further, given that this sample might be a unique group because it was forensic psychiatric sample and was rated using only file information, the likelihood of it being erroneously identified as a taxon has been raised (Lilenfeld, 1998). Since then, several researchers have found no evidence to support the taxonomic structure of the PCL-R (Edens, Marcus, Lilenfeld, \& Poythress, 2006) or the PPI (Marcus, John, \& Edens, 2004).

Skilling, Quinsey, and Craig (2001) further sought to determine whether persistent antisocial behaviour is underlain by a taxon. The authors conducted a study on 1,111 noncustodial boys ranging in grades from 4 to 8 (average age was 11.8). The participants were measured using an 18-item modified PCL (Forth et al., 1990), the Childhood and Adolescent Taxon Scale (CATS; Quinsey, Harris, Rice, \& Cormier, 2006), and the DSM-IV (American Psychiatric Association, 1994) to identify conduct disorder symptoms. This study revealed that all three measures yielded a strong evidence of a taxon. This suggests that among certain young boys who engage in antisocial behaviour, a distinct subgroup can be identified (Skilling et al., 2001).

Extending the research conducted by Skilling et al. (2001), Murrie and colleagues (2007) explored the latent structure among a sample of 757 male youths involved with the juvenile justice system. Using both the PCL:YV and the Antisocial Process Screening Device (APSD; Frick \& Hare, 2001), the results revealed that the PCL:YV and the APSD have a dimensional latent structure among male adolescents. These findings suggest that differences in psychopathic 
traits among adolescents differ in degree, not in kind (Murrie et al., 2007). Edens, Marcus and Vaughn (2011) further examined the taxonomy amongst youth with psychopathic traits by exploring the results of the APSD and the Psychopathic Personality Inventory Short-Form (PPISF; Lilienfeld \& Andrews, 1996). This study found that similar to Murrie et al. (2007) that psychopathic traits, as measured by the two above noted assessment tools, converged on a dimensional model, indicating that psychopathic traits in youth do not represent a latent taxon. This implies that psychopathic features in youth follow a continuum in that some youth possess more psychopathic traits than others and that it is not appropriate to state that a youth is a psychopath or not a psychopath (Edens et al., 2011).

\section{Challenges with Measuring Psychopathic Traits in Youth}

Stability of Youth Psychopathy. Given that some researchers have argued that adolescence is a time of tremendous developmental change (Edens et al., 2001), the need to determine whether certain traits are normative or reflective of an underlying immutable personality, researchers have begun to study the stability of psychopathic traits in youth. Lee, Klaver, Hart, Moretti, and Douglas (2009) explored this with a sample of 83 male, adolescent offenders. Lee and colleagues found a moderate stability of psychopathic traits across six months when both clinical (PCL:YV) and self-report measures (APSD) were utilized. A G coefficient of .75 was found for total PCL:YV scores which is consistent with stability estimates found in adult samples (Alterman, Cacciola, \& Rutherford, 1993; Rutherford, Cacciola, Alterman, \& McKay, 1996). A G coefficient of .72 was obtained for total APSD score which is again, consistent with pervious stability estimates of the APSD (Frick, Kimonis, Dandreaux, \& Farrell, 2003; Lynam \& Gudonis, 2005). Further, it has been argued (Forth \& Book, 2007) that if psychopathic traits are part of the normal adolescent development, then a negative association should be found between 
age at time of assessment and psychopathic features. This has not been demonstrated (Corrado, Vincent, Hart, \& Cohen, 2004; Forth et al., 2003; Murrie, Cornell, Kaplan, McConville, \& LevyElkon, 2004; O'Neill, Lidz, \& Heilburn, 2003). This is evidence that younger adolescents are not more or less psychopathic than their older counterparts (Forth \& Book, 2007), thus providing further indirect evidence of the stability of psychopathic characteristics in adolescence.

Lynam, Caspi, Moffitt, Loeber, and Stouthamer-Loeber (2007) followed 271 individuals who were assessed at age 13 with the Childhood Psychopathy Scale (Lynam, 1997) and then reinterviewed the individuals between nine and 13 years later using the PCL:SV (Hart, Cox, \& Hare, 1995). The study revealed moderate association between psychopathy scores at age 13 and total PCL:SV $(r=.31)$, factor 3 scores $(r=.28)$ and factor 4 scores $(r=.33) 11$ years later into adulthood, and low association between CPS scores and factor 1 scores $(r=.19)$ and factor 2 scores $(r=.15)$. Further, Lynam et al. (2007) examined the relative stability across time by exploring if psychopathic scores at age 13 could predict a diagnosis of psychopathy at age 24 . The authors found moderate stability evidence that were, in terms of diagnostic accuracy, had specificity and negative predictive power. Specificity means the conditional probability that an individual was low on psychopathy at age 13 given that he was not psychopathic at age 24 , while negative predictive power (not having psychopathy at age 24 when assessed as low at age 13) was better than positive predictive power (receiving a diagnosis at age 24 when assessed as high at age 13), but this may be a result of low base rates of psychopathy.

In a further study that examined stability of psychopathic traits, Loney, Taylor, Butler, and Iacono (2007) looked at the stability of externalizing symptoms associated with psychopathy (e.g., conduct problems, hyperactivity/impulsivity, substance use) problems from adolescents into adulthood. The authors found support for moderate stability in antisocial tendencies and 
behavioural features of psychopathy. Both the Antisocial and Detachment subscale of the Minnesota Temperament Inventory (MTI; Loney, Taylor, Butler, \& Iacono, 2002) had concurrent and prospective associations with various externalizing behaviour difficulties. Further, the study revealed that the transition into adulthood is seen with a decrease in psychopathy scores and the magnitude of reduction was greater for antisocial tendencies $(d=$ $.67)$ than for affective deficiencies $(d=.49)$. This suggests that as adolescents mature into adulthood, there is a decrease in antisocial behaviour. This result, in conjunction with the finding that antisocial behaviour declines as the youth transition into adulthood, implies that the variance in both psychopathy subscales is stable across the transition into adulthood despite the overall tendency for attenuation of the antisocial behaviour. Loney et al. (2007) suggested that this provides evidence that psychopathic tendencies in youth should not be viewed as transitory and as normative developmental tendencies but rather as features that may predict severe and potentially life-course persistent antisocial behaviour.

Frick and colleagues (2003) studied the stability of psychopathic traits in a sample of non-referred children in the third, fourth, sixth and seventh grades (at first assessment) and followed them over a 4-year period. These researchers found high stability for both parent and individual reports for the individual subscales of the APSD (Frick \& Hare, 2001). Impulsivity had an intraclass correlation coefficient (ICC) of .87 and the narcissism subscale had an ICC of .92 across the 4-year span. Further, the parent rating for callous/unemotional traits over 4-years was .71 and .80 for total APSD score indicating a substantial degree of stability in parent ratings.

Frick and colleagues (2003) concluded that the findings of their study suggest that parental ratings of psychopathic traits in youth were comparable or even higher than those reported with adults. Further, the stability estimates of parents were comparable to other stability 
estimates of other psychopathological traits in children. These findings have several implications. It is suggestive that psychopathy stability estimates are reliable and it helps to determine whether parents vary over time in their ratings or whether they are influenced by stable perceptions that do not necessary reflect a time change in the child's adjustment. However, the authors also stressed that although the stability estimates obtained in their study were high, they also noted that there was some variability in the level of these traits across time. The data indicated that some youth who had high psychopathic traits improved and showed less traits over time and that youth who had low levels of psychopathic traits initially were less likely to develop these traits later on in life. This has implications for both treatment and for the impact protective factors may have on youth who show high levels of psychopathic tendencies and how these factors influence youth across the span of their adolescent years.

Larsson, Andershed, and Lichtenstein (2006) examined genetic factors to determine if they could explain the variation in the psychopathic personality. They included 1097 identical and fraternal twins from Sweden and administered the self-report Youth Psychopathic Traits Inventory (YPI; Andershed, Kerr, Stattin, \& Levander, 2002) to provide an index of psychopathic tendencies. This scale has been found to demonstrate three factors: grandiose/manipulative dimension, callous/unemotional dimension, and the impulsive/irresponsible dimension. To determine how much genetic and environmental factors account for the variance in psychopathic personality dimensions, the authors used model fitting techniques to determine which model was the best fit for all three dimensions. The results indicated that genetic factors accounted for $43 \%$ of the callous/unemotional dimension, $51 \%$ of the grandiose/manipulative dimension, and $56 \%$ of the impulsive/irresponsible dimension. 
These results show that genetic factors do play an important role in the expression and possible aetiology of psychopathic tendencies in youth.

In summary, the research literature has illustrated that stability in psychopathic traits, as it relates to youth, is consistent with research on adults indicating that psychopathic traits are relatively stable across the adolescent years (Forth \& Book, 2007, Frick et al., 2003; Lee et al., 2009). Researchers have supported that youth who exhibit early signs of psychopathic traits, may in turn, develop serious and persistent antisocial behaviour across their lifespan (Loney et al., 2007).

Labelling. Another important issue with regard to applying psychopathy to youth is the possible negative consequence of labelling. Lyon and Ogloff (2000) describe psychopathy as a "powerful pejorative diagnostic label" (p. 139), which, in conjunction with the popular belief that psychopathy is relatively untreatable (Skeem, Monahan, \& Mulvey, 2002), has led some to question whether the term should be applied to adolescence (Lynam \& Gudonis, 2005). There are several policy implications of using psychopathy assessments with youth involved in the criminal justice system. Studies have shown that such assessments have been used in transferring youth to adult court (Edens et al., 2001; Penney \& Moretti, 2005; Vincent \& Hart, 2002, Zinger \& Forth, 1998), sentencing (Edens et al., 2001), and treatment decisions (D'Silva, Duggan, \& McCarthy, 2004; Lyon \& Ogloff, 2000). However, others argue that the key to successful treatment is the application of proper assessment tools that measure psychopathic characteristics (Lynam \& Gudonis, 2005). From their point-of-view, treatment of psychopathy "will be the most effective earlier in the life course, before negative consequences have accumulated and when the opportunity exists to intervene in multiple areas (e.g., schools, family, peers, and individually)" (Lynam \& Gudonis, 2005, p. 401). 
The research literature pertaining to labelling of psychopathic traits amongst adolescents has demonstrated that applying the psychopathic label and underlying traits to youth results in more punitive decisions from probation officers, potential jurors, juvenile justice judges and clinicians (Boccaccini, Murrie, Clark, \& Cornell, 2008; Murrie, Cornell, \& McCoy, 2005; Rockett, Murrie, \& Boccaccini, 2007). Further, these studies have shown that those with psychopathic traits were more likely to receive harsher sentence recommendations and be given higher ratings of risk. When treatment amenability studies were examined, some indicated that labels had no negative effects (Boccaccini et al., 2008; Murrie et al., 2008; Rockett et al., 2007) whereas others demonstrated that psychopathic labelling contributes to more negative beliefs regarding treatment (Chauhan, Reppucci, \& Burnette, 2007; Edens, Colwell, Desforges, \& Fernandez, 2005; Jones \& Cauffman, 2008).

The term psychopathy has been demonstrated to have several consequences, often of a negative nature. Future clinicians, researchers and those in applied settings should be cognizant of such consequences especially when youth are potentially impacted by such labels.

Developmental Perspective. One explanation in understanding adolescent psychopathy comes from the principles of developmental personality psychopathology (Forth \& Book, 2007). Multifinality and equifinality (Cicchetti \& Rogosch, 1996) have important implications for understanding adolescent psychopathy. Multifinality asserts that individuals may share similar traits but may have different life outcomes. This implies that individuals will vary on their respective risk and protective factors that will influence their development of psychopathy. The concept of equifinality proposes that a common outcome can result from multiple pathways. This implies that diverse processes across different individuals might result in the manifestation 
of psychopathic tendencies in youth (Forth \& Book, 2007). Further research is needed to determine which pathway leads to the development of psychopathy.

A further consideration that must be addressed is whether psychopathy might manifest differently in youth than in adults (Hart, Watt, \& Vincent, 2002). Two important concepts have been examined in this vein: homotypic and heterotypic continuity. Homotypic continuity refers to "the identical behavioural expression of an underlying process across different developmental periods (Cicchetti \& Rogosch, 2002, p. 13); whereas heterotypic continuity means "the manifestations of the same underlying process through different behavioural presentations at different developmental periods" (Cicchetti \& Rogosch, 1996, p. 13). This implies that one must consider the age-appropriate manifestation of psychopathic traits when assessing children, adults and the elderly (Forth \& Book, 2007).

In summary, it is widely accepted that adolescence is a period of tremendous developmental growth. This period is marked by normative behaviour that is often antisocial in nature (Moffitt, 1993). Therefore, the stability of personality traits often receives criticism from researchers who purport that such traits are amendable to change (Edens et al., 2001). Further to this, labelling a youth as having certain personality traits, such as psychopathy, has been demonstrated to have implications in subsequent decisions pertaining to several elements of the criminal justice system. Couple this with the different developmental perspectives as to how psychopathy develops in children and adolescents, lends one to use extreme caution when applying this construct to youth.

\section{Risk of Violence}

In the criminal justice field, valid risk assessment tools play a key role in assessing not only risk for violence and criminal offending but also for preventing recidivism (Howell, 2003) 
and determining appropriate sanctions and rehabilitative services (Schwalbe, 2007). Several types of risk assessments have evolved over the years. The first generation risk assessments were based upon clinical judgements that were given without the aid of structured assessment devices. These types of assessments were weak in their prediction and classification of offenders (Grove $\&$ Meehl, 1996). The next two generations of risk assessments were greatly influenced and structured around statistical associations between the risk assessment tool and repeat offending. The second generation tools, known as actuarial tools or static assessments, were developed based upon evidence regarding the predictive and classification abilities of static factors and how they relate to recidivism (Andrews \& Bonta, 2010). A major criticism of this type of assessment tool was that it does not take into account other risk factors known to be associated with criminal offending (e.g., antisocial peers and attitudes) and other dynamic factors that influence subsequent offending.

The third generation of risk assessments are known as risk/need scales (Andrews \& Bonta, 2010) as they incorporate both static and dynamic factors. One such scale, the Level of Service Inventory-Revised (LSI-R; Andrews \& Bonta, 1995) draws upon both risk factors and criminogenic needs. This scale has demonstrated adequate predictive validity for both general $(r$ $=.36)$ and violent $(r=.25)$ recidivism. Lastly, the fourth generation of risk assessments incorporates both static and dynamic factors with structured professional judgements. These assessment tools are designed to assist clinicians in determining the level of risk management that is required, in which contexts, and at what points in time (Vincent, 2006).

One such tool that utilizes structured professional judgement that has been developed is the Structured Assessment of Violence Risk in Youth (SAVRY; Borum et al., 2006). This scale encompasses several factors known to contribute to violence in youth, such as adverse family 
conditions, community and peer factors, and personal dispositional features, such as early antisocial behaviour. The SAVRY was developed to assist clinicians in evaluating the risk for violence in an adolescent population (Borum et al., 2006). However, a distinguishing feature of this scale is that it incorporates six protective factors that have been shown to reduce the likelihood of violent behaviour, such as prosocial involvement, strong social support and strong commitment to school.

Risk assessment in youth is challenging as adolescence is a time of tremendous developmental change (Edens et al., 2001) with social and environmental factors playing a significant role in how these changes are influenced (Gammelgard, Koivisto, Evonen, \& Kaltiala-Heino, 2008). Risk factors in youth are mainly dynamic, therefore long-term prediction is often limited amongst youth, more so than seen with adults whose environment and personality traits are more stable (Borum et al., 2006; Seagrave \& Grisso, 2002). Despite these challenges, researchers have demonstrated the reliability and validity of applying the SAVRY with both institutionalized (Borum, Bartel, \& Forth, 2002; Dolan \& Rennie, 2008; Gammelgard et al., 2008) and community based samples (Catchpole \& Gretton, 2003; Chapman, Desai, Falzer \& Borum, 2006), among ethnically diverse samples (Chapman et al., 2006) and equally across boys and girls (Gammelgard et al., 2008; Penney, Lee, \& Moretti, 2010) for both non-violent and violent offending (Schmidt, Campbell, \& Houlding, 2011; Welsh, Schmidt, McKinnon, Chattha, \& Meyers, 2008).

Recently, Singh, Grann, and Fazel (2011) undertook a systematic review of nine commonly used violence risk assessments. The authors collected information from 88 independent studies, which were comprised of 25,980 participants, to assess each scale's ability to predict a future event of violence. The study revealed that the SAVRY produced the highest 
rate of predictive validity. The study further illustrated that there was substantial differences between the predictive validity across the nine risk instruments. In violence prediction, the study found that the range of diagnostic odds ratios were from 1.2 to 7.9 , thereby suggesting that the selection of appropriate measures for violence prediction does indeed matter.

In summary, risk assessments have evolved from clinical assessments, which demonstrated poor predictive validity, to actuarial scales and risk/need scales, which showed improved predictive validity, to lastly, structured professional judgments which have illustrated the most promising predictive validity when determining future risk. The SAVRY is one example of a structured professional judgement tool which enables researchers and clinicians to determine the level of risk for violent behaviour amongst an adolescent population. This tool has been demonstrated to have adequate reliability and validity in institutionalized and community samples, among ethnically diverse samples and equally across gender.

\section{Criminal Behaviour - Theory and Developmental Perspective}

Several theories have been postulated as a means of explaining why certain individuals engage in criminal behaviour while others do not. Gottfredson and Hirschi (1990) argued that criminal behaviour results from a deficiency in self-control. They argued that personality traits are not responsible for criminal offending and rather a lack of self-control is the general cause of crime. They wrote that people who have high self-control consider the long-term consequences of their actions while those with low self-control do not. Gottfredson and Hirschi (1990) believe that self-control is learned early in life and once learned, is highly resistant to change. Their theory argues that factors such as quality of relationships with family members, commitment to school or work, and involvement in structured recreational activities mediate criminal activity 
and that changing social factors contribute to subsequent criminal offending (Paternoster, Dean, Piquero, Maxerolle, \& Brame, 1997).

On the other hand, proponents of social learning theory believe that both individual and social factors affect whether an individual will engage in criminal activity (Andrews \& Bonta, 2010). Andrews and Bonta (2010) write that according to social learning theory, people who engage in criminal offending examine the rewards and consequences of such behaviour. If a person perceives a criminal situation as rewarding through their learning history, attitudes towards antisocial behaviour, or through other social factors such as provisions of rewards from family and peers, then criminal offending is likely to occur.

One of the most influential theorists pertaining to youth criminality has been Terrie Moffitt. Moffitt (1993) has wrote that youth criminality can be viewed as belonging to one of two pathways: adolescent limited (AL) and life-course persistent (LCP). For children who take the path of LCP, the age of onset of criminal behaviour can often be traced to a very young age, sometimes even as young as age 3. Moffitt (1993) wrote "across the life course, these individuals exhibit changing manifestations of antisocial behaviour: biting and hitting at age four, shoplifting and truancy at age ten, selling drugs and stealing cars at age sixteen, robbery and rape at age twenty-two, and fraud and child-abuse at age thirty" (p. 679).

Many of the LCP offenders exhibit neurological problems as well, such as difficult temperaments as infants, attention deficit disorders or hyperactivity as children and learning problems in later school years (Moffitt, 1993). These problems are also evident in adulthood, manifested in judgement and problem solving difficulties. Life-course persistent individuals are found to commit a wide range of aggressive acts over their lifetime. Coie, Dodge, and Kupersmith (1990) found that youth who engage in these types of behaviour are often rejected 
and avoided by their peers and parents which can lead them to missing key developmental skills by learning appropriate interpersonal skills.

In contrast with LCP individuals are those who begin their criminal careers in adolescence but stop offending usually by their $18^{\text {th }}$ birthday. The behaviours of the adolescent limited offender are often influenced by peer and social environmental factors and offending is often temporary. These types of offenders are the vast majority of young offenders. It has been estimated that less than $10 \%$ of males show the extreme antisocial behaviour of the LCP (Moffitt, 1993) and less than 2\% of females exhibit this pathway (Coid, 2003). Moffitt (1993) estimated that the majority of adolescents are involved in antisocial behaviour of some nature, but as the responsibilities of young adulthood approach, most cease this behaviour altogether. Moffitt, Caspi, Dickson, Silva, and Stanton (1996) demonstrated that although the life-course trajectories of the two pathways differ, the offending frequency and severity in the teen years is quite similar for the two groups. The offending patterns of the adolescent limited and life-course persistent groups appear to be highly similar during the teen years.

Some researchers have more recently argued that the two pathway model may not give the most comprehensive picture of adolescent offending (Donnellan, Ge, \& Wenk, 2000). D’Unger, Land, McCall, and Nagin (1998) identified four pathways that may better reflect the offending patterns of adolescents. These include the adolescent limited and the life-course persistent, but also include the low-level chronic offenders and a non-offending pattern. Adolescent limited individuals were found to show the pattern described by Moffitt (1993) in that they begin offending in their early teens and decline by early adulthood after peaking around age 16 (Nagin, Farrington, \& Moffitt, 1995). The low-chronic offenders showed an increase in offending through early adolescence, reached a plateau in late teen-hood and remained at this 
level past early adulthood. The life-course persistent youth began at an early age and remained at a high level throughout their lifetime (D’Unger et al., 1998; Nagin et al., 1995; Nagin \& Land, 1993).

As part of the longitudinal Cambridge Study, Farrington et al. (2006) examined indices of life success outcomes for those at age 32 and 48 among three groups of male offenders: persisters (those who were convicted both and after the age of 21), desisters (those who were convicted only before the age of 21) and late-onset offenders (those who were convicted only after the age of 21). Analyses revealed that there was an improvement in life success across all three groups when they were compared from ages 32 and 48 . Those who persisted in offending were the least successful over time, followed by the late-onset offenders and desisters. Desisters were similar to un-convicted men in life success at age 48 but not at age 32 (Farrington et al., 2006).

In summary, several theories have been formulated to explain criminal behaviour. Some theorists purport that criminal behaviour is a result of a lack of self-control (Gottfredson \& Hirschi, 1990), whereas others believe it is a result of social learning (Andrews \& Bonta, 2010). However, Moffitt (1993) has been the most influential in this field in arguing that criminal behaviour in adolescence follows, typically, one of two pathways. The first pathway is believed to be limited to the adolescent period, while the other pathway follows a lifelong trajectory. The latter theory has received considerable attention and validation from several research studies.

\section{Desistence From Offending - Role of Protective Factors}

Most of the research literature on criminal recidivism has focused on factors that relate to the persistence of criminal offending. One of the most predictive factors relating to the persistence of criminal offending has been the identification of antisocial conduct at an early age 
(Loeber, 1996; Loeber \& Farrington, 2000; Moffitt, 1993). However, little research has been conducted on why some people continue on their criminal career paths while others do not. Some authors have written that focusing on risk or deficit-based factors precludes the full understanding of the aetiology of criminal behaviour (Sorbello, Eccleston, Ward \& Jones, 2002; Ward \& Brown, 2004).

In some cases, the focus on external protective factors has demonstrated that having a strong marital commitment (Horney, Osgood, \& Marshall, 1995) and having an attachment to employment goals (Blokland \& Nieuwbeerta, 2005) was associated with criminal desistence. Other research has identified cognitive processes that underlie a cessation or attenuation in criminal behaviour such as having a high self-efficacy in one's ability to desist from crime, beliefs in support of criminal desistence and the ability to identify factors that are linked with a prosocial life (Lloyd, 2007).

The understanding of the link between protective factors and juvenile offending is in its early stage. Some research is focused on understanding certain predispositional factors (e.g. positive temperament), whereas others are attempting to identify genetic-based factors that can buffer criminal outcomes (Masten, 2001; Moffitt, 2005). Loeber, Pardini, Stouthamer-Loeber, and Raine (2007) stated that there were two general perspectives explaining why some people persist in their criminal offending while others desist. The first theory states that criminal desisters are basically the inverse of persisters, meaning that a decrease in the exposure to risk factors will decrease the frequency of offending. The second train of thought states that desistence in criminal offending is the result of increased positive or promotive factors. Loeber et al. (2007) also make the distinction between promotive and protective factors. Promotive factors are defined as factors that are predictive of the low probability of future criminal 
offending in the general population and predict desistence in delinquent populations. Protective factors, on the other hand, are factors that explain why some individuals who, after high exposure to risk factors, still do not become deviant.

Researchers have found several possible explanations as to why some people desist from criminal activity. The first theory was proposed by Glueck and Glueck in 1940. They stated that individuals mature mentally and physically and as such, naturally desist from offending. Gottfredson and Hirschi (1990) stated that criminal offending naturally declines with age due to the normative processes of transitioning from adolescence into adulthood. They put forth the social control theory (Gottfredson \& Hirschi, 1990) that states that people naturally decrease from offending through the formation of social controls or bonds with others. Through changes in life circumstances, such as engaging in romantic relationships, may lead some people away from crime (Sampson \& Laub, 1993). In another theory known as social learning theory, states that the same principle that holds for criminal offending also applies to desistence. The theory holds that low scores on factors relating to the initiation into crime would relate to why some people cease their criminal offending (Loeber et al., 2007), whereas Moffitt (1993) purported that adolescents undergo developmental changes that influence their identity and it is through cognitive, physiological, and sociological changes in one's identity that influences future offending.

When exploring delinquency or antisocial behaviour, many researchers and theorists have explored risk factors associated with these behaviours (Hoge, Andrews, \& Leschield, 1996). However, several researchers have begun to explore other factors that may influence the likelihood that these behaviours do not occur. Protective, promotive or resilient factors have not received as much attention as have risk factors associated with the increase in antisocial 
behaviours (Stouthamer-Loeber, Loeber, Farrington, \& Zhang, 1993), but several studies have begun to explore this issue as it relates to juvenile offending. Hoge et al. (1996) examined three risk factors (family relationship problems, problems of parenting, and parental problems) and four protective factors (positive peer relations, good school performance, participation in organized leisure activities and positive response to authority) on two outcome measures (rates of reoffending and compliance with conditions of their disposition).

Their study revealed family relationship problems and family structuring problems were both significantly related with both outcome variables. Higher problems in these two risk factors indicated higher levels of re-offending and lower compliance ratings on their conditions of disposition. Further, all four protective factors were found to be significantly related to both come measures as well, with higher protective ratings associated with lower levels of offending and more positive adjustment ratings with compliance. Interestingly, however, when Hoge et al. (1996) conducted logistic regressions, for the younger offenders (aged 12 to 14), none of the protective factors were related to the outcome measures. These associations were only significant for youth aged 15 to 17 years.

Hoge et al. (1996) further found that several protective factors reduced the incidence of negative outcomes in the presence of the risk factors. This has important implications for both assessment and intervention activities. This finding implies that assessments should examine not only risk factors but areas of strength as well. Further, when planning intervention strategies with youth offenders, attention should be given to enhancing factors that will help the youth deal with negative factors, especially when these factors are not amenable to change (Hoge et al., 1996). 
Hart, O’Toole, Price-Sharps, and Shaffer (2007) examined both risk and protective factors of violent juvenile offending. They recruited 124 youth and administered seven selfreport measures to obtain indices on family factors, parenting styles, school factors, substance abuse, peer relations, attitudes and beliefs relating to violence, and involvement in extracurricular activities. Hart et al. (2007) compared three groups: non-delinquents, non-violent delinquents, and violent delinquents. Using a discriminant function analysis, they found that substance use, feelings of power and safety relating to violence and guns, grade point average (GPA), aggressive response to shame, caring adults in the community, learning difficulties, and parental demandingness were the best predictors of distinguishing the three groups. The nondelinquents were found to have low scores on the risk factors and high on the protective factors, such as academic achievement, parenting styles, attitudes unfavourable towards violence, having a mentor, positive relationships with peers and being involved in extracurricular activities, whereas the violent delinquent group were the opposite. Non-violent delinquents were found to score in the middle of these two other groups. Furthermore, non-delinquents were found to have significantly more protective factors than the non-violent delinquent group and the violent delinquent group. The non-violent delinquents and violent delinquents did not significantly differ on the number of protective factors. No significant differences were found on the number of risk factors among the three groups (Hart et al., 2007).

Loeber et al. (2007) explored several cognitive, physiological, and psychosocial risk and promotive factors in a sample of boys who were followed from age 7 until age 20 to determine if such factors were related to desistence or persistence in delinquency among adolescent males. The authors did not find any of these risk factors to be predictive of desistence from delinquency. Also, none of the promotive factors were related to desistence from delinquency. Their results 
showed that promotive factors buffer the impact of risk factors, but that risk factors tend to prevail.

These findings, although somewhat contradictory, show that more examination into protective factors relating to antisocial behaviour is warranted. Further, limited research is found in the interplay between promotive or resiliency factors and how these interact with several known risk factors. Caprara, Barbaranelli, and Pastorelli (2001) argue that "prosocial behaviour can also be a powerful antidote to aggression when earlier emotional vulnerability and negative interpersonal experiences, through rejection and stigmatization, lead to hostility, disengagement, and social withdrawal" (p. 201).

In determining the role protective factors have in the desistence of violent offending among violent youth offenders in the Netherlands, Lodewijks, de Ruiter, and Doreleijers (2010) used three subscales (static, dynamic and protective) of the SAVRY to examine the accuracy of the prediction of violence following pre-trial assessment, institutional violence, and violence following release. The results indicated that the static scale had no predictive power across any of the outcomes; however both the dynamic and protective scales were predictive of violent reoffending across all three types of outcome. When protective factors were examined individually, having a strong social support and strong attachment to prosocial adults were significantly predictive of desistence. The authors concluded that protective factors might help mitigate the influence of risk factors in high-risk youth.

Rennie and Dolan (2010) prospectively followed 135 male adolescents to determine the role of protective factors as assessed by the SAVRY in offending desistence among those in custody in the United Kingdom. The results indicated that those who had higher number of protective factors were older at the time of their first arrest and had less non-violent and general 
offences during their follow-up period. Further, the protective score was predictive of desistence from offending. This led the authors to conclude that it appeared that resilient personality traits (i.e., above average intellectual abilities and cognitive skills) may buffer the effects of risk factors, which has major implications on risk management and intervention strategies for highrisk youth.

Serin and Lloyd (2009) conclude that researchers often examine either how an offender began his/her criminal career or how the offender has given up his/her criminal lifestyle, but little attention has been focused on the transition between these two behaviours. They argued that to fully understand both the active offender and the desisted offender, we need to understand the phase between the two and uncover the critical elements needed for this transition. Serin and Lloyd (2009) further suggest that it is believed that offender change may be the result of gradual influences as opposed to instantaneous factors. For offenders, this change may be the most influential when prosocial habits are incorporated into daily activities. There is evidence to suggest that those who desist from crime also engage in other positive life activities that occur in conjunction with desistence, such as substance abuse recovery, gaining employment, and having strong interpersonal commitments (Stouthamer-Loeber, Wei, Loeber, \& Masten, 2004). Serin and Lloyd (2009) state that "the challenge of the offender within transition faces is not simply identifying and changing a single, key risk factor but rather nurturing in multiple life areas a dedication to doing what is necessary to stay crime free" (p. 351).

In summary, over the past few decades, interest into the youth psychopathy concept has focused on developing measures to study the symptoms of the disorder (Forth et al., 2003; Frick \& Hare, 2001; Lynam, 1996), the appropriateness of applying this concept with youth (Salekin \& Lochman, 2008), the temporal stability of this disorder (Frick et al., 2003) and the association 
between psychopathy and antisocial behaviour in adulthood (Gretton et al., 2004). However, it has only been in the past few years that an interest in protective factors as it relates to youth psychopathy has garnered much attention. According to Salekin and Lochman (2008) researchers should examine factors associated with the temporal stability of psychopathic features to identify factors that may be related to the manner in which youth develop prosocial ways and thus may be related to the desistence of criminal offending. If by identifying factors that protect against the stability of psychopathic features, it may help with possible intervention strategies that may lead to personality characteristics that ensure that persistent antisocial behaviour does not continue throughout the lifespan. This has implications for both the individual and society.

\section{Correlates Associated with Youth Psychopathic Traits}

Criminal Behaviour. Increasingly, evidence suggests that there is a strong link between criminal offending and psychopathy, including chronic offending and violent behaviour (Hare et al., 2000). Kosson, Cyterski, Steuerwald, Neumann, and Walker-Matthews (2002) reported a significant relation was found between psychopathy ratings and indices of criminal behaviour, including violent $(r=.27)$ and non-violent offences $(r=.35)$, participation in different types of illegal activity $(r=.45)$, and a willingness to use weapons $(r=.34)$. In a similar study, Farrington (1992) followed 441 individuals from age 8 and found that almost $40 \%$ were convicted of at least one offence by age 32. Interestingly, of those who had committed at least once offence, $6 \%$ were responsible for committing over half of all offences. Farrington also noted that those who were found to be chronic offenders were more likely to have begun committing offences at a young age. 
Violent Offending. Psychopaths are found to be among the most prolific, versatile, and violent of all offenders. Research has found that adult male offenders who were assessed as psychopathic were found to be more likely to have committed a greater variety of crimes, as well as, more offences of any type when compared with non-psychopathic offenders (Porter, Birt, \& Boer, 2001; Hare, McPherson, \& Forth, 1988). Further, psychopathic individuals who commit violent acts do so in a different manner from other offenders. In adult samples, when compared with non-psychopaths, those who were assessed as having high psychopathic traits were more likely to commit violent crimes for instrumental purposes (Cornell et al., 1996; Williamson, Hare, \& Wong, 1987). Woodworth and Porter (2002) found that nearly all homicides (93\%) in their sample were committed by psychopaths for instrumental reasons, whereas only $48 \%$ of non-psychopaths committed homicide for instrumental purposes. Flight and Forth (2007) have demonstrated this relationship in a sample of incarcerated youth. A significant relationship between psychopathy scores and instrumental violence $(r=.59)$ indicating that youth who had high psychopathy trait score were also more likely to engage in instrumental violence, as opposed to reactive violence.

According to Hart and Dempster (1997), there are at least three potential mechanisms that may link violence with psychopathy. The first pertains to antisocial cognitions. Hart and Dempster (1997) stated that psychopaths were more likely to have antisocial thoughts, fantasies and urges and believe these cognitions were normal. Secondly, psychopaths are found to have deficits in affect, meaning they do not process certain feelings in the same manner as nonpsychopaths. Given that affect usually inhibits the expression of violence in non-psychopaths (Patrick \& Zempolich, 1998), psychopaths may commit more acts of violence due to their affective deficits and prominent narcissistic traits (Hare, 1996). Lastly, due to behavioural 
mechanisms, psychopaths are known to be impulsive, which predisposes them to committing all sorts of behaviour including violence before processing their internal cognitions and affective cues (Fritz, Wiklund, Koposov, af Klinteberg, \& Ruchkin, 2008).

Fritz and colleagues (2008) examined 250 Russian male youth offenders to determine whether the APSD (Frick \& Hare, 2001) would be able to discriminate aggressive traits. The results revealed that those who were more violent (based on self-report) were also more likely to score high on psychopathic traits. When youth where were divided on violence (high versus low) based upon the type of crime committed, high ratings of psychopathic traits (based on staff ratings) were also found. These findings lend support that psychopathic youth were more likely to engage in violent criminal offences.

Conduct Disorder. Conduct Disorder (CD) refers to the persistent display of antisocial behaviour that is not normative to the child's development level (Salekin, Rogers, \& Machin, 2001). Although some suggest CD and psychopathy are two parallel disorders (Salekin et al., 2001), others argue that psychopathy is a distinct syndrome that is separate from the behavioural based features of CD (Forth \& Burke, 1998). Forth (1995) found that all youth offenders who scored high on PCL:YV met the diagnosis of CD, while only $30 \%$ of youth with CD were scored as having many psychopathic traits. This suggests that the two disorders, as with adult psychopathy and antisocial personality disorder, are two distinct but overlapping disorders and should not be used interchangeably (Forth \& Burke, 1998).

The relationship between youth who exhibit conduct disorder symptoms and psychopathy has been examined in several studies. Smith, Gacono and Kaufman (1997) found that of 48 male adolescents with conduct disorder, $58 \%$ of those also classified as having high PCL:YV scores (29 or more), had a diagnosis of childhood onset CD. Those in the non-psychopathic group 
(scores of 20 or less) were only found to have childhood onset CD in $10 \%$ of the cases. The authors found a prevalence rating for CD of $66 \%, 51 \%$, and $21 \%$ for those assessed as high, medium, and low on the psychopathy ratings, respectively.

Loeber, Brinthaupt, and Green (1990) examined 210 boys between the grades of 4 and 10 and classified the sample into four categories: ADHD and CD, ADHD only, CD only, and children without ADHD or CD. Loeber et al. (1990) found that those boys who had dual diagnosis of ADHD and CD were more likely to have (after 5 year follow-up) subsequent police contacts (54\%), multiple offences (31\% vs. $21 \%$ for CD only group), higher rates of offending ( $54 \%$ vs. $48 \%$ of CD group), higher self-reported delinquency (62\% vs. $21 \%$ for CD group), higher self-reported aggression (54\% vs. $29 \%$ of CD group), and higher self-reported thefts ( $54 \%$ vs. $29 \%$ of CD group).

Sevecke, Kosson, and Krischer (2009) explored the link between psychopathy, CD and ADHD symptoms in a sample of adolescent detainees. The researchers examined whether they would find that CD and ADHD symptoms would be associated with psychopathic features. They also wanted to test whether the CD-psychopathy link would be stronger than the ADHDpsychopathy link. As predicted, the authors found that childhood conduct problems were a powerful predictor of psychopathy scores in both boys and girls and that the link between ADHD and psychopathy was the result of the large overlap between ADHD and CD symptoms.

Antisocial Behaviour. Vincent, Vitacco, Grisso, and Corrado (2003) argued that the emphasis of past research on behavioural explanations of childhood chronic antisocial behaviour has ignored other factors that play a pivotal role in identifying those who exhibit symptoms of severe antisocial behaviour. Through a cluster analysis of 259 incarcerated youth, Vincent and colleagues (2003) identified four distinct juvenile subtypes on the basis of their affective, 
behavioural, and interpersonal features. Those youth who had the most severe and chronic antisocial behaviours were those who exhibited deficits in all three factors. These findings suggest that the identification of youth who are high at risk for being identified as psychopathic adults needs to take into account all three dimensions of behaviour, interpersonal and affective features. By focusing on behavioural features only, Vincent et al. (2003) argue that a high rate of false positives would occur.

Family Factors. A classic review into the environmental correlates of psychopathy was conducted by McCord and McCord in 1964. They wrote that psychopathy-like traits were associated with early emotional depravation from parental neglect and erratic punishment. In a more recent study, Weiler and Widom (1996) found that those who were abused or neglected as children were more likely to score high on the PCL-R as adults than those who were not victimized as children. Marshall and Cooke (1999) explored family correlates by examining if differences would be observed between those scoring low versus high on the PCL-R. Results revealed that those who were deemed high on the scale were more likely to have problematic childhoods characterized by poor discipline/supervision, parental antipathy, child antipathy to parents, and emotional abuse and neglect. Further, family factors were found to have been more associated with factor 1 (interpersonal/affective) scores, whereas societal influences were more related factor 2 (antisocial lifestyle/behaviour) scores. These studies suggest that early childhood exposure to a negative family environment may play a pivotal role in the development of psychopathic traits in adults.

Forth and Burke (1998) explored family characteristics with a sample of 106 incarcerated male adolescents and 50 male adolescents from the community. The authors found that in both samples, negative family background factors were associated with psychopathic traits. Further, in 
both samples, the strongest association was between total PCL:YV score and parental deviance $(r=.50$ in community sample and $r=.25$ in offender sample). Forth and Burke (1998) further concluded that having a criminal and drug abusing parent, being separated from parents as a child, and experiencing inconsistent discipline may play a causal role in the development of psychopathy. In line with this evidence, Campbell, Porter, and Santor (2004) found that PCL:YV scores were significantly related to history of placement in foster care/alternative living arrangement $(r=.29)$. Kosson et al. (2002) also found that higher PCL:YV scores were negatively related to adolescents' ratings of closeness to their family $(r=-.23)$. O'Neill et al. (2003) found that childhood abuse and neglect was moderately related to Factor 1 (interpersonal/affective), Factor 2 (antisocial lifestyle/behaviour) and total PCL:YV scores. These studies all suggest that negative childhood experiences and parental abuse may be a contributing factor in the development of psychopathy.

Although Hare (1993) once stated that there is very little evidence to support that attachment problems are linked to psychopathy, Farrington (2007) noted that specific parental behaviours were related to the development of psychopathic features in children. Blair, Mitchell, and Blair (2005) purported that attachment problems may be more of a consequence of the emotional dysfunction of the psychopath and that impairments in emotional learning may be a contributing factor to a disrupted attachment process. Further to this debate, Rygaard (1998) wrote that insufficient attachment and the loss of early maternal care were important factors in the development of psychopathy. Rygaard further noted that consistent early contact between mother and child has important implications for normal development of personality and social skills. 
Farrington (2007) stated that parents who show rejection, antisocial behaviour, erratic discipline, and poor supervision can influence the development of psychopathy. Coercive parenting styles have been found to become integrated into a child's behavioural repertoire as early as 24 months. Farrington also noted that certain family factors have been shown to be related to the strong prediction of delinquency or antisocial behaviour. These include: antisocial parents, being male, having low socio-economic status, being impulsive and having poor concentration.

In summary, there appears to be significant support that negative family factors plays an important role in the development of psychopathy (Campbell et al., 2004; Farrington, 2007; Forth \& Burke, 1998; Kosson et al., 2002; O’Neill et al., 2003). Negative affective and behavioural indices are all linked with the manifestation of psychopathic traits in youth.

Academic Achievement. Farrington (1989) noted that youth who have low commitment and low interest in attending school were more likely to commit future acts of violence and criminal offending. Several longitudinal studies have shown that youth who engage in criminal offending tend to have slightly lower IQ's when compared with youth who do not engage in delinquent acts (Hinshaw, 1992; Lynam, Moffitt, \& Stouthamer-Loeber, 1993; Maquin \& Loeber, 1996). Stattin and Magnusson (1995) suggested that IQ, learning problems, and antisocial behaviour are more related to youth who are early-onset offenders rather than lateonset offenders. Brandt, Kennedy, Patrick and Curtin (1992) found a significant association between Factor 2 scores on the PCL-R and lower academic achievement, $r=-.23$ in a sample consisting of 130 male adolescents who were residents of a training facility for severe juvenile delinquency. Campbell et al., (2004) also found an association between PCL:YV scores and 
history of academic behaviour problems $(r=-.31)$ and having ever been expelled from school $(r$ $=.21)$.

The above noted studies consistently illustrate that those with psychopathic traits are less likely to have strong attachments to school achievement, and more likely to have lower IQs, more learning problems and academic behaviour problems.

Antisocial Peers and Associates. Deviant peer relationships have been demonstrated to be associated with psychopathic personality (Forth et al., 2003; Hare, 2003). During adolescence, peer relationships are an integral part of development as they allow adolescents to learn social skills (Laursen, Finkelstein, \& Betts, 2001). It is therefore important to examine the influence peers have when a person exhibits symptoms (i.e., shallow affect, superficial charm, insincerity) that would prevent meaningful and prosocial relationships to occur and explore how deviant peer relationships influence someone with psychopathic personality.

There have been relatively few studies conducted that have examined the influence of peer relationships as they relate to the development and stability of psychopathy in adolescence (Barry, Barry, Deming, \& Lochman, 2008). However, recent research has demonstrated that peer rejection, low social preference and association with deviant peers are all associated with antisocial behaviour and delinquency (Hinshaw \& Lee, 2003; Lochman \& Wayland, 1994; Pardini, Barry, Barth, Lochman, \& Wells, 2006). Moreover, developmental theorists have recently begun to explore whether associating with deviant peers and peer rejection influence the etiology and developmental progression of antisocial behaviour and psychopathy (Kimonis, Frick, \& Barry, 2004). Piatigorsky and Hinshaw (2004) explored the relation between peer rejection and psychopathic traits with a sample of boys with and without ADHD symptoms. They measured psychopathic characteristics with the California Child Q-Set (CCQ; Block, 1961) 
and found that psychopathic traits positively predicted peer rejection even though no relationship was found between psychopathic traits and peer popularity.

Barry et al. (2008) explored whether social relationship variables moderated the stability of psychopathy over time. Examining a sample of moderately aggressive children, the researchers explored several social relationship variables (i.e., social competence, social preference among peers). They found evidence that psychopathic characteristics are stable over time when both teacher and parent ratings were examined. Given the findings of stability, it is not surprising that social relationship variables did not moderate change over time. This implies that psychopathic characteristics were influenced more from previously established interpersonal and affective styles than by current social relationships. Nevertheless, the study provides additional evidence of the association between psychopathic characteristics and social relationships that are characterized by aggressiveness and deviant behavioural patterns.

Muñoz, Kerr, and Besic (2008) examined the quality of relationships with peers among a community sample of 12 to 15 year old adolescents who were either high or low on psychopathic traits. Their findings suggest that regardless of psychopathic traits, youth in this study were able to have important peers in their life. Further, these peer relationships were reciprocal, meaning that if they named a specific peer, that person also rated the relationship as important. One interesting finding, however was that youth high on psychopathic traits were more likely to be found to have less stable peer relationships, meaning they were less likely to keep the same friends from one year to the next.

Youth with high psychopathic traits are often observed with several other behavioural and social correlates. These youth are more likely to engage in criminal behaviour that is often of a violent nature and they are more likely to exhibit conduct disorder and ADHD symptoms. 
These youth are characterized by their antisocial tendencies and often come from neglected and abused homes. The lack of parental discipline and erratic behaviour on the part of the parent has been demonstrated to be linked with the characteristics of youth psychopathy. Coupled with the high percentage of youth with these traits to have problems in school, both academically and behaviourally, further increases their experience with antisocial inclinations. These youth often align themselves with other antisocial youth which has been demonstrated to exacerbate antisocial and aggressive behavioural patterns.

\section{Predictive Validity of Risk Assessments in Youth - PCL:YV and SAVRY}

In a recent review, Edens, Campbell, and Weir (2007), conducted a meta-analysis as a means of addressing the limitations of prior research that had been conducted on psychopathy and recidivism among youth samples. Edens et al. (2007) wanted to build on the largely narrative reviews previously published on the association between psychopathy and recidivism. Therefore, their analysis was conducted on 21 published and unpublished studies that examined the PCL:YV ratings with three outcome measures: general, violent and sexual recidivism. Their analyses revealed a total, non-redundant sample of 2,867 cases available for follow-up. The mean PCL:YV score varied widely $(M=20.5$, range 9-28) and the average follow-up period across studies was 31.75 months (range 3-120 months). In terms of criterion measures, general recidivism was able to be coded from all 21 studies, violent recidivism from 15 studies, and sexual recidivism from four studies.

The results of this meta-analysis revealed that the mean weighted effect sizes $\left(r_{w}\right)$ for the PCL total scores were .26 for general recidivism, .23 for violent recidivism, and .07 for sexual recidivism. Only the latter was non-significant. However, very large Q statistics were obtained for all three outcome measures indicating a large degree of variability among the effects. When 
outliers were removed from the dataset, the effect sizes were found to be .24 for general recidivism, .25 for violent recidivism, and .07 for sexual recidivism. Of note, Factor 2 (antisocial lifestyle/behavioural) was found to have a stronger correlation with general recidivism than Factor 1 (interpersonal/affective) $\left(r_{w}=.29\right.$ vs. $\left.r_{w}=.18\right)$ although their CIs overlapped slightly.

This meta-analysis revealed that, akin to adult studies, a significant relationship exists between psychopathy and general and violent recidivism among male adolescents. This study also showed a substantial variability in the effect sizes among the studies. This suggests that there is a high probability of possible moderators accounting for the variability obtained between studies. It is therefore necessary for future studies to examine if other factors, in conjunction with psychopathy, are influencing criminal re-offending (Edens et al., 2007).

In another large meta-analysis examining all the PCL measures (PCL-R, Hare, 2003; PCL:YV, Forth et al., 2003 and the PCL:SV, Hart, Cox, \& Hare, 1995), Leistico et al., (2008) found that psychopathy scores were equally predictive of criminal recidivism and institutional infractions for both youth and adult offending. More specifically, the impulsive and antisocial behavioural traits (Factor 2) was found to be a stronger predictor of criminal behaviour than the affective and interpersonal traits factor (Factor 1). When follow-up times were examined, Factor 2 scores (impulsive and antisocial behaviour) were better predicting long-term follow-up offences than studies which had a short follow-up period. Olver and colleagues (2009) also conducted a meta-analysis of the PCL:YV (Forth et al., 2003), the Level of Service/Case Management Inventory (YLS/CMI, Andrews, Bonta, \& Wormith, 2004), and the SAVRY (Borum et al., 2006) and found support that all three measures were able to significantly predict 
general, non-violent and violent recidivism. The authors noted however that youth predictive studies tended to have shorter follow-up periods than comparable studies conducted on adults. Cauffman and colleagues (2009) conducted a multi-method approach study to examine the predictive validity of three measures of juvenile psychopathy: the PCL:YV (Forth et al., 2003), the YPI (Andershed et al., 2002) and the NEO Psychopathy Resemblance Index (NEO PRI; Lynam \& Widiger, 2007) and found that the PCL:YV was able to predict recidivism at 6 months but not for recidivism at 36 months. This is suggestive that the PCL:YV may be more accurate when predicting proximal events and show a gradual attenuation in the predictive validity of recidivism (Cauffman et al., 2009). This may be evidence of possible divergent pathways in criminal offending in youth. Some young offenders with psychopathic traits maintain these traits over time and continue to lead an antisocial lifestyle, whereas others become less psychopathic as they age. This has major implications on treatment amenability, sentencing, court diversion and release decisions and the potential role of protective factors with young offenders.

In summary, psychopathic assessments and risk of violence measures appear to have adequate validity in respect to predicting general, non-violent and violent recidivism. In terms of short versus long-term prediction, the studies conducted thus far are suggestive that the PCL:YV may be more accurate in predicting proximal events and that factors which may explain why some youth do not go on to commit future offences warrants further research.

\section{The Current Study}

Consistently, we have seen in the literature a strong association between psychopathy and serious repetitive crime, often of a violent nature (Hare, 1996; Hare et al., 2000; Hemphill et al., 1998). As seen in the adult literature, those who are found to possess psychopathic 
characteristics are more likely to require a disproportionate amount of resources from the criminal justice system (Forth \& Book, 2007). Therefore, it is imperative that we identify youth who have similar characteristics as they are more likely than other offenders to become persistent, high-rate serious adult offenders (Forth \& Book, 2007). Furthermore, of equal interest, are those youth who are found to possess psychopathic traits but do not go on to have negative outcomes. Protective factors have not received any attention as it relates to psychopathy in youth and the research on resilience or protective factors in relation to juvenile delinquency desistence is limited at best.

The objective of the present study was to examine three main research questions. First, do youth offenders who persist in their offending differ from youth who do not recidivate on correlates of antisocial behaviour? Towards this end, comparisons between persisters and desisters on various clinical and psychosocial (i.e., substance abuse, family background, criminal history) was evaluated. These factors were based on self-report information as well as file-based information, when possible. Second, were risk assessments that measure the construct of youth psychopathy and risk for violence in adolescence able to predict future criminal offending? More specifically, were each risk assessment instrument independently and collectively able to predict the imminence and probability of general, non-violent, violent and sexual offending for shortterm and long-term offending? Lastly, the third objective of the present study was to determine if certain factors were associated with the desistence of criminal offending. This examination explored whether protective factors influence the effects of certain risk factors and subsequent criminal behaviour. To achieve these objectives, several hypotheses were generated: 
1. Youth who persist in offending will differ significantly from those who do not persist on factors associated with antisocial behaviour. More specifically, persisters will more likely have these factors present whereas desisters will not;

2. it is expected that PCL:YV total scores will show predictive validity for non-violent and violent convictions and that Factor 4 (antisocial) of the PCL:YV will also be associated with non-violent and violent recidivism. Exploration into the predictive validity of the other factors will be assessed;

3. it is hypothesized that SAVRY risk ratings will be predictive of non-violent and violent offending in this sample but not predictive of sexual offending;

4. it is expected that youth who score high on the PCL:YV and the SAVRY risk score will commit non-violent and violent offences more quickly than those scoring low on psychopathy and risk of violence; and

5. lastly, to examine if factors can identify youth who desist from criminal offending or who persist in their criminal careers, items that have been demonstrated to provide protection against risk factors will be examined to determine if such factors alone, or how they interact with certain risk factors (psychopathy, risk for violence), can predict group classification on type of re-offending (non-violently, violently, or sexually). Given the limited research currently on protective factors, exploratory analyses will be conducted to determine the nature of such associations. 


\section{Chapter II: Method}

\section{Participants}

Selection Criteria. Participants for the present study were all initially assessed as part of three separate studies from which consent was given for additional follow-up studies. All participants were either remanded or incarcerated in youth detention centres at the time of the original studies. Participants were approached and asked to participate in a study examining risk factors relating to criminal behaviour. Recruitment posters were displayed in each of the institutions and offenders were interviewed on the basis of this recruitment strategy. The first sample (sample 1) was assessed between November, 1985 and December, 1988 and consisted of 75 male young offenders from Willingdon Youth Detention Centre in British Columbia. The second sample (sample 2) consisted of 106 male young offenders from Ottawa-Carleton Detention Centre (OCDC) and Sprucedale Youth Centre in Ontario that were assessed between February, 1994 and December, 1994. The last sample (sample 3) contained 60 male offenders, assessed between September, 2003 and March, 2004 from Brookside Youth Centre, OCDC and Bluewater Youth Centre in Ontario. Six of the participants were dropped from the study due to incomplete file information which did not allow for coding of several outcome variables, leaving a total sample of 235 youth.

Demographics. Descriptive statistics for this sample are shown in Table 2. The ethnic composition of this study was primarily Caucasian (76.1\%), with a smaller minority being Aboriginal (14.4\%) and 9.5\% classified as other ethnicities. The average age of the participants was 17.1 years $(S D=1.1)$ and the mean grade level achieved for this sample was $8.9(S D=1.4)$. Time spent in custody ranged from 1 day to 2446 days $(M=193.2, S D=204.4$ days $)$. The majority of the sample (79.6\%) consisted of offenders who had criminal records for non-violent 
Table 2

Descriptive Statistics of Total Sample

\begin{tabular}{|c|c|c|c|}
\hline Variable & $M(S D)$ & $\%(n / 235)$ & Range \\
\hline Age at assessment & $17.1(1.1)$ & & $13-20$ \\
\hline Sentence length (days) & $193.2(204.4)$ & & $1-2446$ \\
\hline \multicolumn{4}{|l|}{ Ethnicity } \\
\hline Caucasian & & $76.1(175)$ & \\
\hline Black & & $4.8(11)$ & \\
\hline Aboriginal & & $14.4(33)$ & \\
\hline Hispanic & & $1.3(3)$ & \\
\hline Asian & & $1.7(4)$ & \\
\hline Other & & $1.7(4)$ & \\
\hline Education & $8.9(1.4)$ & & $4-12$ \\
\hline
\end{tabular}

Note: $M=$ mean, $S D=$ standard deviation

offences prior to their index offence. Although $40.3 \%$ had prior official convictions for violent offences, $87.6 \%$ of youth admitted to having committed a prior violent offence. Two percent of participants had official prior convictions of sexual offences. For the index offence, the sample consisted of offenders who had committed non-violent offences $(82.1 \%)$, violent offences $(38.7 \%)$, and sexual offences $(7.2 \%)^{2}$.

\section{Measures}

Psychopathy. The PCL:YV (Forth et al., 2003) was developed from the Hare Psychopathy Checklist - Revised (Hare, 1991) and uses the same methodology to assess psychopathic traits in adolescents. Information was gathered using a semi-structured interview

\footnotetext{
${ }^{2}$ Note that percentages do not add to $100 \%$ as offenders could have convictions in more than one category
} 
and collateral file review format. Raters assigned a participant a scores of 0 (definitely does not apply), 1 (somewhat applies) or 2 (definitely applies) for the 20 items that comprise the scale.

The scores were then totalled to provide an overall psychopathy score. To determine if the total PCL:YV score (20-item) would differ from the 13-item (3-factor model) or the 18-item (4-factor model) on outcome prediction, total scores for each of the 13-item and 18-item scale were computed as well. The scores on the PCL:YV were further grouped into low, moderate and high scoring groups. The cut-off ranges for the high scoring group was scores equal to or above 30 , with the low scoring groups consisting of those who scored below 20 and the moderate scoring group having a range of 20 to 30 on the PCL:YV. These scores were as recommended in the PCL:YV manual (Forth et al., 2003).

Similar to the PCL-R (Hare, 2003) the PCL:YV assesses interpersonal, affective, behavioural and antisocial features to provide a composite assessment of psychopathic traits. Studies in which the PCL:YV has been used has revealed high inter-rater reliability scores, as well as high internal consistency ( $\alpha=.85$ to .94$)$ for both institutional and community samples. This scale has also been demonstrated to have adequate concurrent validity (Forth et al., 2003; Skeem \& Cauffman, 2003). In comparison with a 20 -item parent and teacher rating scale of the APSD (Frick \& Hare, 2001) with the PCL:YV, scores on both measures have been reported to correlate at .35 when file ratings of the PCL:YV were obtained and .53 when interview and file information was gathered. Gretton et al. (2004) found that PCL:YV total scores correlated moderately with number of conduct disorder symptoms (see Appendix A for item descriptions).

Risk of Violence. The SAVRY (Structured Assessment of Violence Risk in Youth; Borum et al., 2006) is a 24 -item risk scale developed to assess the level of risk for violence in youth. The scale consists of ten historical risk factor items, eight social/contextual factor items, 
and eight individual risk factor items. This scale also incorporates six protective factors that have been demonstrated to buffer the risk of delinquency and aggression (Caprara et al., 2001; Hoge et al., 1996). All of the risk items are rated on a 3-point scale (low, moderate, and high) and the protective factor items are rated as either present or absent. Risk scores on the SAVRY of 0,1 and 2 correspond to having no difficulties, having moderate difficulties, or having significant difficulties. For research purposes, these numbers were transformed into numerical values and then summed to provide an overall risk score. The range of this scale is from zero to 48. Given the SAVRY manual does not provide cut-off score guidelines for distinguishing low, moderate, and high scoring individuals, the criterion used in this study was to divide the groups based on one standard deviation above and below the mean to group low, moderate and high scoring youth. The low scoring group consisted of those youth who had a risk rating below 16.6, the moderate scoring group were those youth who had a risk score between 16.7 and 32.0, whereas the high scoring group were those who scored above 32 . The protective factor ratings were not included in the total risk rating score (see Appendix B for SAVRY item descriptions). The structured professional judgement summary score was not computed for this study given that the SAVRY was retrospectively coded and only file and interview notes were used to score each individual. Therefore, since the purpose of this measure is to provide a structured clinical judgment, the author felt that given the test was retrospectively coded, it was not appropriate to ascribe a clinical judgment rating based on file information.

Borum, Lodewijks, Bartel, and Forth (2010) report high reliability coefficients (.81 to .97) for SAVRY total risk score. Dolan and Rennie (2008) found ICCs of .98 for historical risk factor, .91 for social/contextual factor, .95 for individual risk factor and .97 for total risk. 
Conduct Disorder. Conduct disorder symptoms were examined based upon file and interview information according to the Diagnostic and Statistical Manual of Mental Disorders (DSM-IV, American Psychological Association, 1994). The DSM-IV lists 15 symptoms that are associated with the diagnosis of conduct disorder, with the presence of at least three symptoms required for a diagnosis. Each symptom is coded as either present or absent. Further, each symptom confirmed present must have been present 12 months preceding the assessment, with at least one symptom present within the preceding six months. Moreover, the symptom must have resulted in clinical significant impairment on social, academic or occupational function. Two scores were computed: a total conduct disorder score that encompassed all 15 symptoms, which yielded a score with a range of zero to 15 , and an aggression symptom score that encompassed the seven aggression symptoms (see Appendix C for list of symptoms). All items were retrospectively coded based upon file and interview notes.

Canadian Police Information Centre (CPIC) Records. Official criminal records were obtained from the Royal Canadian Mounted Police (RCMP). Raters were blind to PCL:YV scores. Information on convictions ${ }^{3}$, length in custody, and months free in community were obtained for each youth. Convictions were classified into four categories. Non-violent offences consisted of theft, possession of stolen property, fraud, breaches of conditions of probation, escaping custody, driving offences, offences, prostitution, committing an indecent act in public, public nudity or obscene matter. Violent offences included murder, manslaughter, attempted murder, robbery, kidnapping, possession of a weapon and arson. Further, all weapons related charges were classified as violent offences. Sexual offences included sexual assault, aggravated sexual assault, sexual assault with a weapon, and sexual interference. General offences were

\footnotetext{
${ }^{3}$ All outcome variables are based upon participants having received an official conviction. The term "offences" is used throughout the paper and refers only to convictions and not charges that were dropped or if the participant received an acquittal.
} 
classified as any criminal convictions occurring during the follow-up period. Persistence was defined as any conviction (general, non-violent or violent) that was recorded after release for the index offence. Desistence was defined as the opposite, with no official convictions recorded after release for the index offence (see Appendix D for coding sheet).

Factors Associated with Psychopathy and Risk of Violence. Previous researchers have identified several correlates that are often found with those with psychopathic traits or who are at risk for violence. Through interview data and file information, each youth was examined to determine if they were found to have each correlate. These items were scored as either present or absent. The items were substance abuse problems, ADHD diagnosis, parental neglect, ever have experienced violence in the home, parental criminal history, having been in foster care, having criminal peers or associates, having been rejected by peers, having a low commitment to school, having poor school achievement, having been violent towards another person or animal, ever have used a weapon to injure or threaten someone or in a commission of a crime, and ever have been expelled or suspended from school.

\section{Procedure}

Data Collection. Approval from the Research Ethics Board (REB) at Carleton University was obtained to conduct a follow-up, archival study (see Appendix E). For two of the three samples (1994 and 2004), PCL:YV scores were completed at the time of the original study. For the 1986 sample, the PCL:YV was scored retrospectively based on file information. All three cohorts were scored using the 2003 PCL:YV manual criteria. SAVRY and conduct disorder diagnosis was retrospectively coded for all three samples. Further, criminal offending for prior and index offences were retrospectively coded for all participants. To ensure that a biasing effect 
did not influence PCL:YV, SAVRY and CD coding, all previous assessment scores were kept blind from the author. All measures were coded by the author.

Recidivism data was coded based upon official crime records from the RCMP. The release date for their index offence for each offender was determined by taking the index offence and calculating the number of days corresponding to two-thirds of his sentence. For youth who returned to prison during the follow-up period for offences that were excluded from the type of outcome measured, time spent incarcerated was taken into account. For example, if a youth received a one month sentence for a non-violent offence, when violent recidivism was examined, the time incarcerated for the non-violent offence was subtracted from their total time available for that particular follow-up period. All participants were then followed from the time of this date until the date of their first offence for general, non-violent, violent and sexual offences or until study completion in the absence of recidivism data (October 15, 2009). The maximum follow-up period was 24.1 years ( 8814 days, $M=5437.3$ days, $S D=2366.1$ ). Recidivism was coded as the first official conviction committed during the post-index follow-up period. Each offender was followed for three follow-up periods: one year post release, five years post release and ten years post release. Longer follow-up periods were not included in this study given that only one cohort was available to code beyond 15 years and thus, the sample size would only be based on 75 participants.

Coder Training. Training on the use of the PCL:YV and the SAVRY was provided by one of the authors of each scale (Dr. Forth) to the experimenter. The training sessions included familiarization with the construct of psychopathy and the risk of violence. In addition, practice sessions with mock offenders were provided until adequate agreement in scoring was achieved. Further, twenty percent of the PCL:YV, SAVRY, CD diagnosis, and recidivism data were dually 
coded to allow for the evaluation of inter-rater reliability of each measure. This was achieved through ICCs (McGraw \& Wong, 1996). Internal consistency was also evaluated for each scale and respective factors utilizing Cronbach's alpha for continuous data and Kudar-Richardson for dichotomous data.

\section{Analytical Strategy}

Inter-rater reliability and internal consistency was examined for all measures in this study. For internal consistency, Cronbach's alpha was used in cases of continuous data, whereas the Kuder-Richardson formula was used for dichotomous data. Background information on participants was recorded from file information obtained at the time of the original assessment. Demographic information, attention-deficit hyperactivity diagnosis, familial factors, criminal associates, academic factors, substance abuse, conduct disorder symptoms, pre-assessment offending, psychopathy scores and SAVRY risk and protective ratings were summarized and presented to compare those who persisted in offending non-violently, violently and sexually with those who desisted in offending. Further, comparisons were evaluated on demographics and outcome measures across each sample to determine whether the three samples were homogeneous (see Appendix F).

In line with objective one, those who persisted in offending post index were compared with offenders who did not go on to re-offend on several measures. In cases when the comparisons were made between continuous variables, $t$-tests were computed. When comparisons were evaluated with categorical variables, chi-square tests were performed. Given that multiple comparisons were examined, the Bonferroni correction was applied so that the alpha was not inflated, thus reducing the risk of type I error. 
In line with objective 2 of this dissertation, the predictive validity was examined for the psychopathy ratings, ratings of risk of violence, and number of CD symptoms on future criminal offending amongst the participants. Firstly, base rates were computed for each of the four offence categories and were presented for the overall sample and for each of the three follow-up periods ( 1 year, 5 years, and 10 years). Secondly, point-biserial correlations between PCL:YV total scores (a continuous variable) and recidivism (yes/no) over the entire follow-up period were computed. Similar presentation was conducted for SAVRY risk ratings and the number of CD symptoms.

To examine the predictive validity of each measure, Receiver Operator Characteristic (ROC) curves were analyzed to determine whether the PCL:YV, SAVRY and number of CD symptoms were predictive of future offending. The ROC analyses evaluated whether the PCL:YV, SAVRY and/or CD symptoms were able to predict an event, in this case whether or not the participant committed another offence upon release from custody. These analyses produced an area under the curve (AUC) that determined whether any instrument was predictive, better than chance that someone re-offended. An AUC of 0.0 represents perfect negative prediction, 0.5 refers to no better than chance, and 1.0 reflects perfect prediction. However, in the area of violence risk prediction, Rice and Harris (2005) have suggested that AUCs of .539 be considered low, .639 is moderate, and .714 is said to be in the high range.

Time to first offence was examined by utilizing Cox regression survival analysis techniques with three separate models calculated for each outcome measure (non-violent, violent and sexual). Time to re-offend was calculated using the time to first failure during the prospective follow-up period for each offender in each of the follow-up period bins (1 year, 5 years, and 10 years). The date of first offence was subtracted from the date of release to yield the 
number of days spent free before an offence was committed. In the absence of an offence, the date of 1 year, 5 years or 10 years post index was used to calculate the number of days free without an event. Log-rank tests were examined to determine if the PCL:YV or SAVRY was associated with the imminence of offending for non-violent, violent, and sexual offences. Further, for both assessment tools, Kaplan -Meier survival functions were also examined to describe the time it took for the youth to re-offend for those scoring low, moderate or high on each of the respective scales.

Lastly, to achieve the third objective of this study, risk factors were examined along with protective factors to discern whether each would add incremental validity to the prediction of criminal persistence. To accomplish this, logistic regression analyses were computed to discern whether factors alone or in combination can predict whether a youth will either persist or desist from non-violent, violent or sexual offending. Further, hierarchical logistic regressions were computed to determine the incremental validity of the protective factors assessed by the SAVRY on the prediction of future criminal offending over the PCL:YV and SAVRY risk total scores. These were calculated for each of the outcome periods. 


\section{Chapter III: Results}

\section{Inter-rater Reliability and Internal Consistency}

All study variables were examined utilizing Stata 10.1 to examine data entry accuracy, missing values, and outliers. Inter-rater reliability was measured for PCL:YV total score, each factor score, SAVRY risk and protective ratings, and conduct disorder diagnosis. Forty-five randomly selected participants (compromising of almost $20 \%$ of all participants) were used for the purposes of inter-rater reliability. As illustrated in Table 3, these analyses revealed a very high agreement was obtained for total scores $\left(\mathrm{ICC}_{\mathrm{A}, 1}=.96\right)$ and across each of the factor scores with coefficients ranging from .92 to .97 . SAVRY risk rating was found to have an $\mathrm{ICC}_{\mathrm{A}, 1}$ of .93 , while the factors ranged from .91 to .96 . Conduct disorder agreement was found to have an $\mathrm{ICC}_{\mathrm{A}, 1}$ of .95 . ICCs for the criminal offending variable were also shown to have high agreement, with non-violent offences post-index having an $\mathrm{ICC}_{\mathrm{A}, 1}$ of .96 , violent offences having an $\mathrm{ICC}_{\mathrm{A}, 1}$ of .98, and sexual offences having an $\mathrm{ICC}_{\mathrm{A}, 1}$ of .97 .

Internal reliability was also calculated for each of the PCL:YV factor scales and for the overall PCL:YV score, the SAVRY risk, protective, and factor scores, and for the number of symptoms comprising the diagnosis of conduct disorder for overall sample. The PCL:YV total score was found to have an internal consistency of 0.86 , with the factor scores ranging from 0.61 to 0.76 . For the SAVRY risk rating, the alpha was found to be 0.82 , while the risk factors ranged from 0.48 to 0.71 . The SAVRY protective factor had a reliability coefficient of .67 ; whereas conduct disorder symptoms were found to have an overall consistency of 0.66 (see Table 3 for summary). 
Table 3

Internal Consistency and Inter-Rater Reliability for PCL:YV, SAVRY and Conduct Disorder

Scale

Internal Reliability

$\mathrm{ICC}_{\mathrm{A}, 1}$

PCL:YV total

.86

.96

PCL:YV Factor 1

.72

.92

PCL:YV Factor 2

.76

.95

PCL:YV Factor 3

.60

.94

PCL:YV Factor 4

.70

.97

SAVRY Risk

.82

.93

SAVRY Historical

.64

.96

SAVRY Social

.46

.92

SAVRY Clinical

.71

.91

SAVRY Protective

.67

.92

Conduct Disorder

.66

.95

Note: PCL:YV = Psychopathy Checklist: Youth Version, SAVRY = Structured Assessment of Violence Risk in Youth; ICC $=$ Intra-class correlation coefficient with absolute agreement

\section{Data Screening}

Missing Values. All raw predictor and outcome data variables were examined for accuracy in data entry and missing values. The percentage of missing values for the PCL:YV, SAVRY and CD symptoms were negligible with only the SAVRY score missing one participant's value. Given this, no substitution measures were made to the raw data. 
Data Screening. The PCL:YV, SAVRY and CD symptoms were all examined for univariate outliers, normality (i.e., skewness and kurtosis; see Table 4), linearity and homogeneity of variance. Upon visual inspection of bivariate scatterplots, no serious violations of linearity or homoscedasticity were observed. To examine if all pairs in any regression analysis were related, the author examined the residual plots and again, no violations were noted. There we no outliers beyond $+/-3$ standard deviations of the distributional range, therefore no truncation was required for any variable. There were no extreme cases of skewness or kurtosis and moreover, there were no multivariate outliers as measured by the Mahalonobis distance (critical value $=10.83)$. Therefore, all univariate and multivariate assumptions were tested and tenable.

Table 4

Descriptive Statistics for PCL:YV, SAVRY and CD Symptoms

\begin{tabular}{|c|c|c|c|c|c|}
\hline Variable & $M$ & $S D$ & $\begin{array}{c}\text { Skewness } \\
(\mathrm{SE})\end{array}$ & $\begin{array}{c}\text { Kurtosis } \\
\text { (SE) }\end{array}$ & Range \\
\hline PCL:YV total & 24.8 & 8.0 & $-.56(.16)$ & $-.54(.32)$ & $5-39$ \\
\hline Interpersonal & 3.9 & 2.3 & $.01(.16)$ & $1.04(.32)$ & $0-8$ \\
\hline Affective & 4.9 & 2.3 & $-.42(.16)$ & $-.99(.32)$ & $0-8$ \\
\hline Behavioural & 6.6 & 2.2 & $-.53(.16)$ & $-.23(.32)$ & $0-10$ \\
\hline Antisocial & 7.1 & 2.4 & $-.78(.16)$ & $-.24(.32)$ & $1-10$ \\
\hline SAVRY risk & 24.3 & 7.7 & $-.10(.16)$ & $-.46(.32)$ & $6-43$ \\
\hline Historical & 9.6 & 3.6 & $.16(.16)$ & $-.28(.32)$ & $1-19$ \\
\hline Social & 5.1 & 2.2 & $.32(.16)$ & $-.75(.32)$ & $1-11$ \\
\hline Clinical & 9.6 & 3.4 & $-.26(.16)$ & $-.42(.32)$ & $0-16$ \\
\hline SAVRY Protective & 1.3 & 1.3 & $.99(.16)$ & $.55(.32)$ & $0-6$ \\
\hline CD symptoms & 8.1 & 2.8 & $-.50(.16)$ & $-.44(.32)$ & $0-14$ \\
\hline
\end{tabular}

Note: $\mathrm{M}=$ mean, $\mathrm{SD}=$ standard deviation, $\mathrm{SE}=$ standard error, $\mathrm{PCL}: \mathrm{YV}=$ Psychopathy Checklist: Youth Version, SAVRY $=$ Structured Assessment of Violence Risk in Youth, $C D=$ conduct disorder 


\section{Assessment Summaries}

Psychopathy. The mean scores on the PCL:YV are shown in Table 4. Overall, the sample had a mean PCL:YV score of $24.8(S D=8.0)$ with a range of 5 to 39 . Twenty four percent were found to be in the low range ( 0 to 19.9 ), $40.4 \%$ scored in the moderate range (20 to 29.9 ) and $34.9 \%$ scored in the high range (score of 30 or above) (see Table 4 for factor score summaries).

SAVRY. For SAVRY risk ratings, the mean score for the entire sample was $24.3(S D=$ 8.0) with a range of 6 to 43 . For this scale, $14.9 \%$ scored in the low range ( 0 to 16.6 ), $68.5 \%$ scored in the moderate range (16.7 to 32.0 ), while $16.2 \%$ scored in the high range ( 32.1 and above) (see Table 4 for summary of subscales).

Conduct Disorder. The mean number of conduct disorder symptoms for the entire sample was $8.1(S D=2.8)$ with a range of 0 to 14 . Ninety-seven percent of the youth in this study met the DSM-IV criteria of having a CD diagnosis.

To illustrate the associations between the three predictor measures, Pearson correlation coefficients were computed, which are presented in Table 5. 
Table 5

Correlations between PCL:YV, SAVRY and Conduct Disorder

\begin{tabular}{llllllllllllll} 
Variable & 1 & 2 & 3 & 4 & 5 & 6 & 7 & 8 & 9 & 10 \\
\hline
\end{tabular}

1. PCL:YV total

2. Factor 1

3. Factor 2

4. Factor 3

5. Factor 4

6. SAVRY risk

7. SAVRY historical

8. SAVRY social

9. SAVRY clinical

10. SAVRY protective

11. CD symptoms
$.74 \quad--$

$\begin{array}{lll}.84 & .57 & --\end{array}$

$\begin{array}{rrr}.77 & .41 & .55\end{array}$

$\begin{array}{lll}.74 & .36 & .47\end{array}$

$\begin{array}{lll}.49 & .24 & .34\end{array}$

$.40 \quad .16 \quad .26$

$.19 \quad .06 \quad .10$

$\begin{array}{lll}.55 & .34 & .41\end{array}$

$-.41$

.50
$-.09$

.31

Note: PCL:YV = Psychopathy Checklist: Youth Version, SAVRY = Structured Assessment of Violence Risk in Youth, $\mathrm{CD}=$ conduct disorder; bold denotes significance at the $p<.001$ level.

Prior Offence Characteristics. Age at first known offence (self-reported) was found to be $12.0(S D=2.7)$ with a range of 5 to 18 . Overall, $82.9 \%$ of youth had a pre-assessment history of any conviction, $79.6 \%$ had a non-violent conviction, $40.4 \%$ had a violent conviction, and $2.1 \%$ had a sexual conviction. The three most common types of non-violent offences committed by this sample were property offences $(70.2 \%)$, breach offences $(46.4 \%)$ and minor offences (26.4\%). The most common violent offences were assault (26.0\%), weapon offences (10.6\%) and robbery $(8.5 \%)$. None of the youth had a prior conviction of murder.

Index Offence Characteristics. Examination of the index offences revealed that eightytwo percent of youth were found to been referred or have committed non-violent offences, $38.7 \%$ had been referred or had committed violent offences, and $7.3 \%$ were found to have been referred or committed sexual offences. The average length of incarceration for index offences was 193.2 
$(S D=204.4)$ days with a range of 1 day to 2446.6 days ( 6.7 years). The three most common types of non-violent offences committed by youth were property offences $(68.5 \%)$, breach offences $(46.4 \%)$ and minor offences $(17.5 \%)$. The most common violent offences committed were robbery (18.7\%), assault (16.2\%) and weapon offences (10.2\%).

Base Rates of Criminal Offending. Table 6 illustrates the base rates for general, nonviolent, violent and sexual offences for the entire follow-up period, and for 1 year, 5 years, and 10 years post release. As indicated in the table, almost $80 \%$ of youth had committed any type of offence during the entire follow-up period. Seventy-five percent committed a non-violent offence, $62 \%$ committed at least one violent offence, and $3.8 \%$ committed a sexual offence. Given that few offenders were sexual offenders prior to or at index, the results indicated that only 9 youth had committed another sexual offence during the follow-up period. As a result of the low base rate of sexual recidivism, subsequent analyses with sexual offending as the outcome variable were dropped.

During the 1-year follow-up period, $37.4 \%$ of youth had committed a non-violent offence and $10.6 \%$ had committed a violent offence. In 5 -years, $68.5 \%$ had committed a non-violent crime, while $43 \%$ had committed a violent crime. Of youth who were followed for 10 years (samples 1 and $2, n=178$ ), 69.1\% were found to have committed a non-violent offence and $56.7 \%$ had committed a violent offence.

Youth who scored high on psychopathy showed higher base rates of violent offending (75.6\%), than those scoring in the moderate (55.8\%) or low groups $(53.4 \%), \chi^{2}(2)=9.81, p<$ .006. For non-violent offences, youth in the low range had more convictions $(79.3 \%)$ than those in the high group (76.8\%) and moderate group (72.6\%), however this difference was not statistically significant, $\chi^{2}(2)=.96, p=.62$ (see Figure 1 ). 
Table 6

Base Rates of Offending

\begin{tabular}{lccccc}
\hline Variable & $n$ & General & Non-Violent & Violent & Sexual \\
\hline Overall & 235 & 79.6 & 75.7 & 62.1 & 3.8 \\
1 Year Follow-Up & 235 & 39.1 & 37.4 & 10.6 & 0 \\
5 Year Follow-Up & 235 & 71.9 & 68.5 & 43.0 & 2.1 \\
10 Year Follow-Up & 178 & 73.6 & 69.1 & 56.7 & 3.9 \\
\hline
\end{tabular}

Note: 10 Year follow-up was conducted on Samples 1 and 2 only (1986 and 1994)

Figure 1. Base Rates of Non-Violent and Violent Offending by PCL:YV Groups

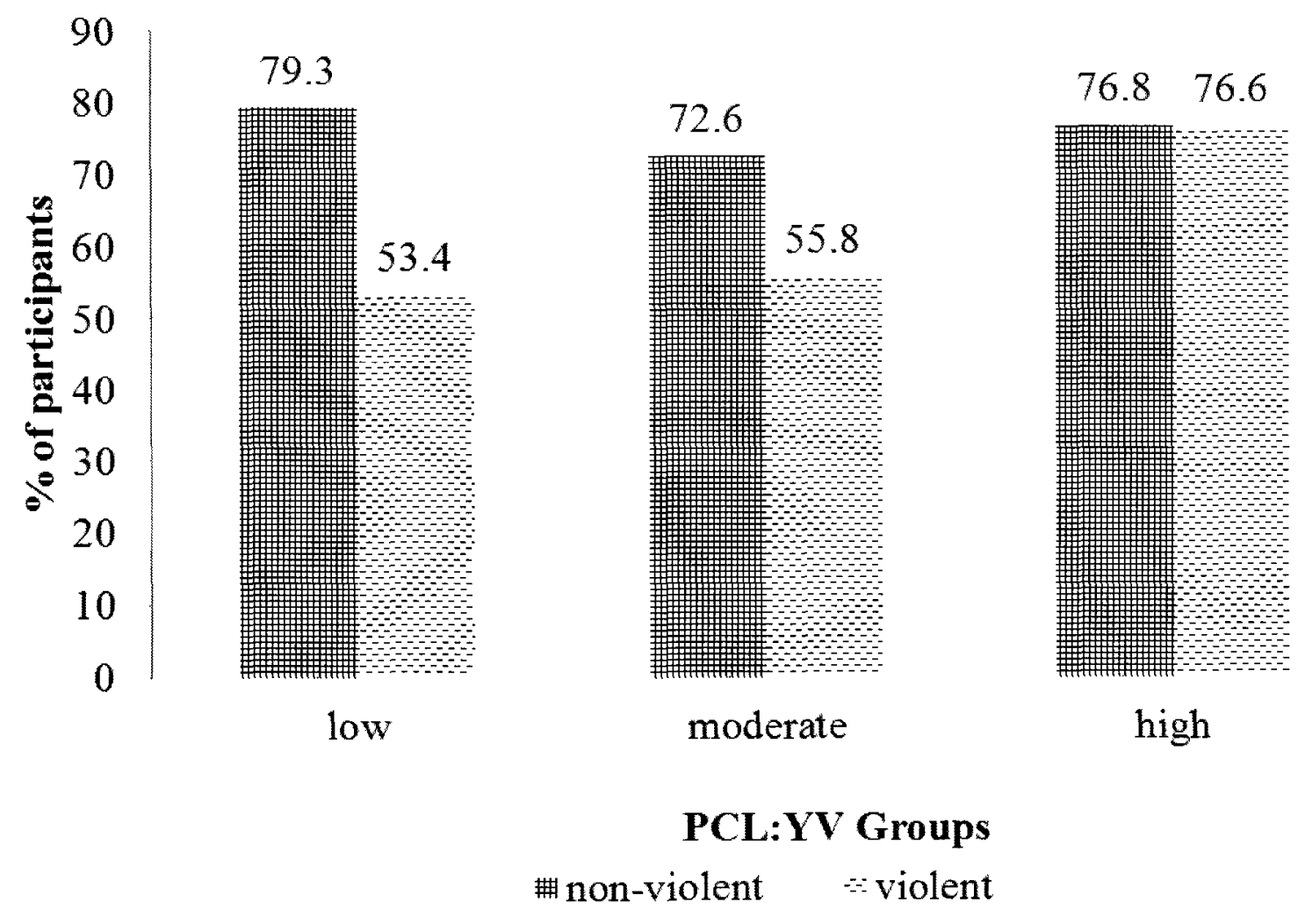


Youth who were grouped as being in the high category on the SAVRY were found to have higher rates of non-violent offences (86.8\%) than those scoring in the low group (60.0\%) or moderate group $(77.0 \%)$, with the chi-square test indicating that the groups significantly differed, $\chi^{2}(2)=7.47, p<.03$. The SAVRY groups also differed on the number of violent offences committed within each group, with those in the high scoring group committing more violent crimes $(81.5 \%)$ than those in the low (37.1\%) or high scoring groups $(63.4 \%), \chi^{2}(2)=15.34, p<$ .001 (see Figure 2).

Figure 2. Base Rates of Non-Violent and Violent Offending by SAVRY groups

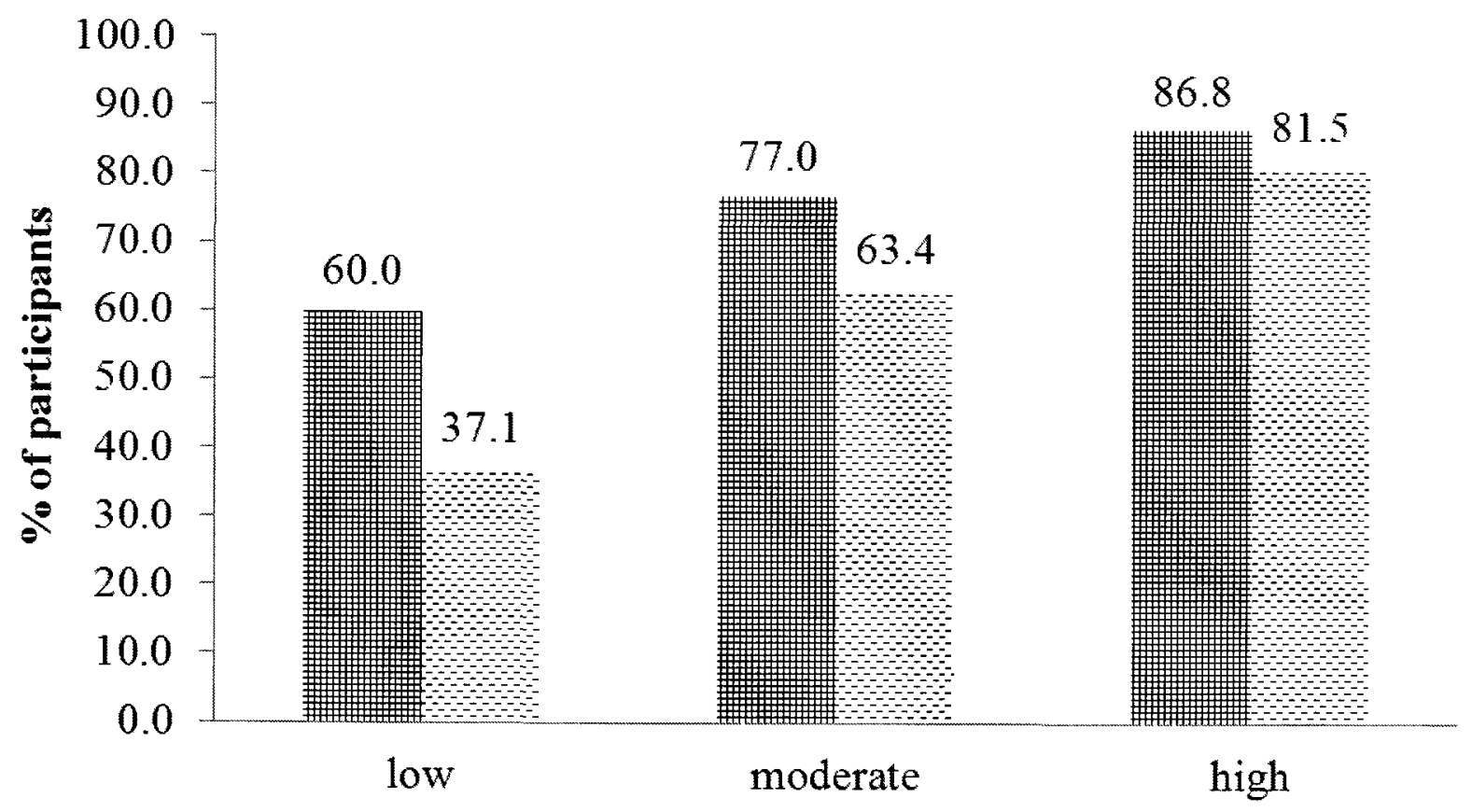

SAVRY Risk Groups

\#non-violent $\approx$ violent 


\section{Comparisons between Persisters and Desisters - Objective One}

To examine hypothesis one, comparisons were made between those who persisted in offending after their release with those who did not go on to re-offend for both non-violent and violent offences. The first set of comparisons examined the PCL:YV total and factor scores, SAVRY total risk and subscales and the number of CD symptoms for youth who either persisted $(n=178)$ or desisted $(n=57)$ from non-violent offending. As presented in table 7, no differences were found at the $p$-value level of .004 or less. Youth who committed non-violent offences during the follow-up period were very similar to youth who did not commit a non-violent offence on the total PCL:YV scores and each of the factor scores. For the SAVRY measure, youth who desisted scored lower on total risk at the $p<.02$ level and had lower protective scores at the $p<.03$ level, but after Bonferroni correction, these comparisons were no longer significant. 
Table 7

Youth who Either Persisted or Desisted From Non-Violent Offending: A Comparison of the PCL:YV, SAVRY and CD Scores

\begin{tabular}{|c|c|c|c|c|}
\hline Variable & $\begin{array}{c}\text { Persisters } \\
M(S D) \\
n=178\end{array}$ & $\begin{array}{c}\text { Desisters } \\
M(S D) \\
n=57\end{array}$ & $t$ & $p$ \\
\hline PCL:YV & $24.8(7.9)$ & $24.6(7.9)$ & -.21 & .83 \\
\hline Interpersonal & $3.8(2.3)$ & $4.0(2.3)$ & .59 & .56 \\
\hline Affective & $4.9(2.4)$ & $5.0(2.2)$ & .38 & .70 \\
\hline Behavioural & $6.7(2.1)$ & $6.4(2.3)$ & -.77 & .44 \\
\hline Antisocial & $7.2(2.3)$ & $6.9(2.5)$ & -.96 & .34 \\
\hline SAVRY & $24.9(7.4)$ & $22.3(8.3)$ & -2.28 & .02 \\
\hline Historical & $9.9(3.5)$ & $8.8(4.1)$ & -1.87 & .06 \\
\hline Social & $5.3(2.3)$ & $4.7(2.0)$ & -1.82 & .07 \\
\hline Clinical & $9.8(3.2)$ & $8.8(3.8)$ & -1.96 & .06 \\
\hline Protective & $1.2(1.3)$ & $1.6(1.4)$ & 2.23 & .03 \\
\hline CD Symptoms & $8.3(2.7)$ & $7.3(3.1)$ & -2.44 & .02 \\
\hline
\end{tabular}

Note: PCL:YV = Psychopathy Checklist: Youth Version; SAVRY = Structured Assessment of Violence Risk in Youth; $\mathrm{CD}=$ conduct disorder; after Bonferroni correction of .05/11 $=p<.004$, none of the above comparisons were significant.

When comparisons were evaluated for youth who persisted $(n=146)$ in violent offending with youth who did not $(n=89)$, several significant results were noted. Firstly, youth who did not recidivate violently were found to have lower PCL:YV total scores $(M=22.7, S D=7.9)$ than youth who persisted to recidivate violently $(M=26.0, S D=7.7), t(233)=-3.11, p<.002$. Further, youth who desisted in violent offending were found to have lower factor 4 (antisocial behaviour) scores, $(M=6.4, S D=2.6)$ than those who went on to commit violent offences post release $(M=7.6, S D=2.1), t(233)=-3.79, p<.001$. Youth who desisted on violent offending were also found to have lower scores on SAVRY risk, $t(232)=-4.48, p<.001$, SAVRY 
historical, $t(232)=-3.55, p<.001$, and SAVRY clinical, $t(232)=-4.63, p<.001$ and have

higher protective scores, $t(232)=4.28, p<.001$ than those who went on to recidivate violently.

Youth who desisted were also more likely to have lower number of CD symptoms, $t(233)=$ -

$3.99, p<.001$ than persisters of violent offending (see Table 8).

Table 8

Youth who Either Persisted or Desisted From Violent Offending: A Comparison of the PCL:YV, SAVRY and CD Scores

\begin{tabular}{|c|c|c|c|c|}
\hline Variable & $\begin{array}{c}\text { Persisters } \\
M(S D) \\
n=146 \\
\end{array}$ & $\begin{array}{c}\text { Desisters } \\
M(S D) \\
n=89 \\
\end{array}$ & $t$ & $p$ \\
\hline PCL:YV & $26.0(7.7)$ & $22.7(7.9)$ & -3.11 & .002 \\
\hline Interpersonal & $4.0(2.4)$ & $3.6(2.1)$ & -1.36 & .18 \\
\hline Affective & $5.2(2.4)$ & $4.5(2.3)$ & -1.99 & .05 \\
\hline Behavioural & $6.9(2.1)$ & $6.1(2.2)$ & -2.81 & .005 \\
\hline Antisocial & $7.6(2.1)$ & $6.4(2.6)$ & -3.79 & .000 \\
\hline SAVRY & $26.0(7.1)$ & $21.5(7.9)$ & -4.48 & .000 \\
\hline Historical & $10.3(3.5)$ & $8.6(3.7)$ & -3.55 & .000 \\
\hline Social & $5.4(2.2)$ & $4.7(2.2)$ & -2.48 & .02 \\
\hline Clinical & $10.3(3.0)$ & $8.3(3.6)$ & -4.63 & .000 \\
\hline Protective & $1.0(1.2)$ & $1.8(1.4)$ & 4.28 & .000 \\
\hline CD Symptoms & $8.6(2.6)$ & $7.1(3.0)$ & -3.99 & .000 \\
\hline
\end{tabular}

Note. PCL:YV = Psychopathy Checklist: Youth Version; SAVRY = Structured Assessment of Violence Risk in Youth; $\mathrm{CD}=$ conduct disorder; bold denotes significance after Bonferroni correction of $p<05 / 11=.004$

Youth who persisted in non-violent offending were found to have committed their first offence at a younger age, $t(232)=3.48, p<.001$ and to have spent fewer days incarcerated for their index offence, $t(233)=3.34, p<.001$ than non-violent desisters (see Table 9). 
Table 9

Youth who Either Persisted or Desisted From Non-Violent Offending: A Comparison of Prior and Index Offences

\begin{tabular}{|c|c|c|c|c|}
\hline Variable & $\begin{array}{c}\text { Persisters } \\
M(S D) \\
n=178\end{array}$ & $\begin{array}{c}\text { Desisters } \\
M(S D) \\
n=57\end{array}$ & $t$ & $p$ \\
\hline \multicolumn{5}{|l|}{ Prior Offences } \\
\hline Non-Violent & $5.6(5.4)$ & $4.4(4.8)$ & -1.47 & .14 \\
\hline Violent & $.72(1.2)$ & $.75(1.7)$ & .15 & .88 \\
\hline Sexual & $.02(.13)$ & $.04(.19)$ & .85 & .40 \\
\hline \multicolumn{5}{|l|}{ Index Offences } \\
\hline Non-Violent & $3.3(3.3)$ & $3.1(4.8)$ & -.44 & .66 \\
\hline Violent & $.80(3.4)$ & $1.3(3.2)$ & .97 & .33 \\
\hline Sexual & $.17(1.7)$ & $.19(.44)$ & .08 & .94 \\
\hline Age at first offence & $11.7(2.6)$ & $13.1(2.7)$ & 3.48 & .001 \\
\hline Num. of days incarcerated & $169.0(123.3)$ & $250.2(240.9)$ & 3.34 & .001 \\
\hline
\end{tabular}

Note: Num. of days incarcerated refers to $2 / 3$ of sentence imposed; bold denotes significance after Bonferroni correction of $p<.05 / 8=.006$

Similarly, those who persisted in violent offending were found to be younger at the age when their first crime was committed, $t(232)=4.24, p<.001$ and have spent fewer days incarcerated for their index offence, $t(233)=2.38, p<.02($ see Table 10$)$. 
Table 10

Youth who Either Persisted or Desisted From Violent Offending: A Comparison of Prior and Index Offences

\begin{tabular}{|c|c|c|c|c|}
\hline Variable & $\begin{array}{c}\text { Persisters } \\
M(S D) \\
n=146 \\
\end{array}$ & $\begin{array}{c}\text { Desisters } \\
M(S D) \\
n=89 \\
\end{array}$ & $t$ & $p$ \\
\hline \multicolumn{5}{|l|}{ Prior Offences } \\
\hline Non-Violent & $5.4(5.3)$ & $5.1(5.2)$ & -1.47 & .14 \\
\hline Violent & $.68(1.1)$ & $.82(1.6)$ & .15 & .88 \\
\hline Sexual & $.02(.14)$ & $.02(.15)$ & .85 & .40 \\
\hline \multicolumn{5}{|l|}{ Index Offences } \\
\hline Non-Violent & $3.2(3.3)$ & $3.1(4.8)$ & -.44 & .66 \\
\hline Violent & $.58(3.4)$ & $1.3(3.2)$ & .97 & .33 \\
\hline Sexual & $.05(1.7)$ & $.19(.44)$ & .08 & .94 \\
\hline Age at first offence & $11.5(2.5)$ & $13.0(2.7)$ & 3.48 & .001 \\
\hline Num. of days incarcerated & $169.1(127.2)$ & $220.8(206.0)$ & 3.34 & .001 \\
\hline
\end{tabular}

Note: Num. of days incarcerated refers to $2 / 3$ of sentence imposed; bold denotes significance after Bonferroni correction of $p<.05 / 8=.006$

In order to assess whether persisters differed from desisters on non-violent offending on correlates of antisocial behaviour, chi-square analyses were conducted. The results revealed that the two groups did not significantly differ from each other on measures of social, personal, familial or academic factors (see Table 11). 
Table 11

Youth who Either Persisted or Desisted From Non-Violent Offending: A Comparison of Clinical,

Social, Familial and Academic Factors

\begin{tabular}{|c|c|c|c|c|}
\hline Variable & $\begin{array}{c}\text { Persisters } \\
\% \\
\end{array}$ & $\begin{array}{c}\text { Desisters } \\
\% \\
\end{array}$ & $\chi^{2}$ & $p$ \\
\hline $\mathrm{CD}$ diagnosis & 97.7 & 92.9 & 3.06 & .08 \\
\hline Substance Abuse & 86.0 & 76.8 & 2.63 & .11 \\
\hline $\mathrm{ADHD}$ diagnosis & 51.2 & 44.2 & .77 & .38 \\
\hline Parental neglect & 59.7 & 55.6 & .29 & .59 \\
\hline Experienced violence & 39.1 & 46.4 & .95 & .33 \\
\hline Parental criminality & 41.3 & 34.0 & .91 & .34 \\
\hline Foster care & 61.0 & 60.7 & .002 & .97 \\
\hline Criminal peers & 96.3 & 92.5 & 1.38 & .24 \\
\hline Peer rejection & 40.5 & 28.8 & 2.27 & .13 \\
\hline Low commit. school & 85.8 & 74.5 & 3.78 & .05 \\
\hline Poor school achievement & 85.3 & 78.6 & 1.42 & .23 \\
\hline Ever violent & 87.6 & 87.7 & .001 & .98 \\
\hline Ever used weapon & 65.9 & 64.9 & .02 & .89 \\
\hline Ever expelled & 58.5 & 49.1 & 1.55 & .21 \\
\hline Ever suspended & 92.6 & 84.2 & 3.56 & .06 \\
\hline
\end{tabular}

Note: $\mathrm{CD}=$ conduct disorder, $\mathrm{ADHD}$ refers to attention deficit hyperactivity disorder; after Bonferroni correction of $.05 / 15=p<.003$, none of the above comparisons were significant.

When youth who recidivated violently were compared with youth who did not on the same antisocial correlates, only two significant differences emerged. Youth who persisted in violent offending were more likely to have substance abuse problems, $\chi^{2}(1)=10.2, p<.001$ when compared with those who desisted, and further, persisters were more likely than desisters to have a low commitment to school, $\chi^{2}(1)=13.9, p<.001$ (see Table 12) 
Table 12

Youth who Either Persisted or Desisted From Violent Offending: A Comparison of Clinical, Social, Familial and Academic Factors

\begin{tabular}{lcccc}
\hline Variable & $\begin{array}{c}\text { Persisters } \\
\%\end{array}$ & $\begin{array}{c}\text { Desisters } \\
\%\end{array}$ & $\chi^{2}$ & $p$ \\
\hline CD diagnosis & 97.9 & 94.3 & 2.16 & .14 \\
Substance Abuse & $\mathbf{8 9 . 7}$ & $\mathbf{7 3 . 9}$ & $\mathbf{1 0 . 1 6}$ & $\mathbf{. 0 0 1}$ \\
ADHD diagnosis & 53.2 & 43.4 & 2.02 & .16 \\
Parental neglect & 59.7 & 57.0 & .17 & .68 \\
Experienced violence & 43.0 & 37.5 & .67 & .41 \\
Parental criminality & 45.0 & 30.6 & 4.59 & .03 \\
Foster care & 62.8 & 58.0 & .53 & .47 \\
Criminal peers & 97.0 & 92.9 & 2.00 & .16 \\
Peer rejection & 42.3 & 30.0 & 3.20 & .07 \\
Low commit. school & $\mathbf{9 0 . 3}$ & 71.3 & $\mathbf{1 3 . 9 7}$ & $\mathbf{. 0 0 0}$ \\
Poor school achievement & 89.0 & 74.7 & 8.20 & .004 \\
Ever violent & 88.3 & 86.5 & .16 & .69 \\
Ever used weapon & 67.6 & 62.5 & .63 & .43 \\
Ever expelled & 58.3 & 52.8 & .68 & .41 \\
Ever suspended & 93.1 & 86.5 & 2.75 & .10 \\
& & & & \\
\hline
\end{tabular}

Note: $\mathrm{CD}=$ conduct disorder, $\mathrm{ADHD}=$ attention deficit hyperactivity disorder; bold denotes significance after Bonferroni correction of $p<.003$

\section{Role of Risk Factors in the Prediction of Criminal Offending - Objective Two}

Receiver Operator Characteristic. With respect to the prediction of future offending, Receiver Operator Characteristics (ROC) analyses were computed. First, analyses were computed to determine if the PCL:YV, SAVRY or the number of CD symptoms could predict if any offence was committed during the entire follow-up period. Table 13 shows that the none of the PCL:YV total scores (20-item, 13-item or 18-item) nor any of the PCL:YV factor scores were predictive of general recidivism (AUCs ranged from .48 to .55 ). SAVRY risk score was significantly predictive of general recidivism $(\mathrm{AUC}=.61, p<.05)$, however this is considered to 
be in the low-moderate range. The SAVRY clinical score was also significantly predictive of general offending, $\mathrm{AUC}=.62, p<.05$, but again this is considered to be in the low-moderate range. The number of $\mathrm{CD}$ symptoms was also found to be in the low-moderate range, $\mathrm{AUC}=$ $.60, p<.05)$ of predictive accuracy. When the protective score was analyzed to predict desistence, it was found to be significant at the $p<.01$ level, with an AUC of .63.

\section{Table 13}

Predictive Accuracy (ROC) and 95\% Confidence Intervals (CI) for General Recidivism for Entire Follow-Up Period

\begin{tabular}{|c|c|c|c|c|c|}
\hline \multirow[t]{2}{*}{ Variable } & \multirow[t]{2}{*}{$r$} & \multirow[t]{2}{*}{ AUC } & \multirow[t]{2}{*}{$S E$} & \multicolumn{2}{|c|}{ Asymptotic $95 \%$ CI } \\
\hline & & & & Lower & Upper \\
\hline PCL:YV 20 item & .02 & .53 & .05 & .44 & .62 \\
\hline PCL:YV 13 item & -.00 & .51 & .05 & .42 & .60 \\
\hline PCL:YV 18 item & .04 & .54 & .05 & .45 & .63 \\
\hline Factor 1 - interpersonal & -.04 & .48 & .05 & .39 & .57 \\
\hline Factor 2 - affective & .00 & .51 & .05 & .43 & .60 \\
\hline Factor 3 - behavioural & .06 & .55 & .05 & .46 & .64 \\
\hline Factor 4 - antisocial & .07 & .55 & .05 & .45 & .64 \\
\hline SAVRY risk & $.16^{*}$ & $.61 *$ & .05 & .52 & .70 \\
\hline SAVRY historical & .13 & .57 & .05 & .48 & .67 \\
\hline SAVRY social & .12 & .58 & .05 & .49 & .67 \\
\hline SAVRY clinical & $.15^{*}$ & $.62^{*}$ & .05 & .52 & .71 \\
\hline SAVRY protective ${ }^{a}$ & $-.19^{* *}$ & $.63^{* *}$ & .05 & .54 & .72 \\
\hline CD symptoms & .15 & $.60^{*}$ & .05 & .50 & .70 \\
\hline
\end{tabular}

Note: PCL:YV = Psychopathy Checklist: Youth Version, SAVRY = Structured Assessment of Violence Risk in Youth, $\mathrm{CD}=$ conduct disorder; $\mathrm{AUC}=$ area under the curve, $\mathrm{SE}=$ standard error; $\mathrm{a}=$ for the SAVRY protective score, the prediction was for non-offending.

${ }^{*} p<.05,{ }^{* *} p<.01$ 
For short-term follow-up (1-year post release), the PCL:YV total scores were not adequate predictors of general recidivism (AUCs of .54 to .56) and factor 3 (behavioural) reached statistical significance at $p<.05$, however with an AUC of .59, this is considered low. Similarly, SAVRY risk and SAVRY clinical AUCs reached statistical significance of $p<.05$, but with AUCs of .58 and .59 respectively, these were in the low range of accuracy (see Table 14).

Table 14

Predictive Accuracy (ROC) and 95\% Confidence Intervals (CI) for General Recidivism for OneYear Follow-Up Period

\begin{tabular}{|c|c|c|c|c|c|}
\hline \multirow[t]{2}{*}{ Variable } & \multirow[t]{2}{*}{$r$} & \multirow[t]{2}{*}{ AUC } & \multirow[t]{2}{*}{$S E$} & \multicolumn{2}{|c|}{ Asymptotic $95 \%$ CI } \\
\hline & & & & Lower & Upper \\
\hline PCL:YV 20 item & .09 & .56 & .04 & .48 & .63 \\
\hline PCL:YV 13 item & .07 & .54 & .04 & .47 & .62 \\
\hline PCL:YV 18 item & .10 & .56 & .04 & .49 & .63 \\
\hline Factor 1 - interpersonal & -.04 & .48 & .04 & .40 & .55 \\
\hline Factor 2 - affective & .09 & .56 & .04 & .49 & .64 \\
\hline Factor 3 - behavioural & $.15^{*}$ & $.59 *$ & .04 & .51 & .66 \\
\hline Factor 4 - antisocial & .11 & .57 & .04 & .50 & .65 \\
\hline SAVRY risk & $.15^{*}$ & $.58 *$ & .04 & .51 & .65 \\
\hline SAVRY historical & .12 & .57 & .04 & .50 & .65 \\
\hline SAVRY social & .06 & .53 & .04 & .45 & .61 \\
\hline SAVRY clinical & $.16^{*}$ & $.59 *$ & .04 & .52 & .66 \\
\hline SAVRY protective ${ }^{a}$ & $-.14^{*}$ & .57 & .04 & .50 & .65 \\
\hline CD symptoms & .11 & $.58 *$ & .04 & .50 & .67 \\
\hline
\end{tabular}


As shown in Table 15, general recidivism in the 5-year period was not predicted by any of the PCL:YV total scores or any of the factor scores. The SAVRY risk score showed significant prediction at the $p<.001$ level with an AUC of .64 and each subscale was also found to be statistically significant with AUCs ranging from .60 to .63 . Having a high number of CD symptoms was also predictive of general recidivism during the 5-year follow-up period (AUC $=$ $.60, p<.05)$, but was considered to be in the low range.

Table 15

Predictive Accuracy (ROC) and 95\% Confidence Intervals (CI) for General Recidivism for FiveYear Follow-Up Period

\begin{tabular}{|c|c|c|c|c|c|}
\hline \multirow[t]{2}{*}{ Variable } & \multirow[t]{2}{*}{$r$} & \multirow[t]{2}{*}{ AUC } & \multirow[t]{2}{*}{$S E$} & \multicolumn{2}{|c|}{ Asymptotic 95\% CI } \\
\hline & & & & Lower & Upper \\
\hline PCL:YV 20 item & .08 & .56 & .04 & .48 & .64 \\
\hline PCL:YV 13 item & .05 & .54 & .04 & .46 & .62 \\
\hline PCL:YV 18 item & .09 & .56 & .04 & .48 & .65 \\
\hline Factor 1 - interpersonal & -.00 & .51 & .04 & .42 & .59 \\
\hline Factor 2 - affective & .03 & .53 & .04 & .45 & .61 \\
\hline Factor 3 - behavioural & .13 & .58 & .04 & .50 & .66 \\
\hline Factor 4 - antisocial & .12 & .57 & .04 & .48 & .65 \\
\hline SAVRY risk & $.24 * *$ & $.64 * * *$ & .04 & .56 & .72 \\
\hline SAVRY historical & $.21 * *$ & $.62 * *$ & .04 & .54 & .70 \\
\hline SAVRY social & $.15^{*}$ & $.60^{*}$ & .04 & .52 & .68 \\
\hline SAVRY clinical & $.21^{* *}$ & $.63 * *$ & .04 & .55 & .71 \\
\hline SAVRY protective ${ }^{a}$ & $-.20 * *$ & $.63^{* *}$ & .04 & .55 & .71 \\
\hline CD symptoms & $.17^{*}$ & $.60^{*}$ & .04 & .52 & .68 \\
\hline
\end{tabular}


For the 10-year follow up period for the prediction of general recidivism, factor 3 (behavioural) score showed predictive accuracy in the low-moderate range (AUC $=.63, p<.05$ ) and the SAVRY risk score $(\mathrm{AUC}=.63, p<.01)$ and each of the SAVRY subscales (AUCs of .60 to $.62, p<.05)$ showed predictive accuracy in the low to moderate range. The number of CD symptoms was also found to be statistically significant in the prediction of general offending with an AUC of .61, $p<.05$. The highest AUC was obtained for the protective score. When desistence was predicted, the protective score indicated moderate accuracy with an AUC of .67, $p<.001$ (see Table 16). 
Table 16

Predictive Accuracy (ROC) and 95\% Confidence Intervals (CI) for General Recidivism for TenYear Follow-Up Period

\begin{tabular}{|c|c|c|c|c|c|}
\hline \multirow[t]{2}{*}{ Variable } & \multirow[t]{2}{*}{$r$} & \multirow[t]{2}{*}{ AUC } & \multirow[t]{2}{*}{$S E$} & \multicolumn{2}{|c|}{ Asymptotic $95 \% \mathrm{CI}$} \\
\hline & & & & Lower & Upper \\
\hline PCL:YV 20 item & .07 & .56 & .05 & .47 & .66 \\
\hline PCL:YV 13 item & .05 & .54 & .05 & .45 & .63 \\
\hline PCL:YV 18 item & .09 & .57 & .05 & .47 & .66 \\
\hline Factor 1 - interpersonal & -.05 & .48 & .05 & .38 & .57 \\
\hline Factor 2 - affective & -.00 & .51 & .05 & .42 & .62 \\
\hline Factor 3 - behavioural & $.19^{*}$ & $.62 *$ & .05 & .53 & .72 \\
\hline Factor 4 - antisocial & .14 & .59 & .05 & .49 & .68 \\
\hline SAVRY risk & $.22^{* *}$ & $.63 * *$ & .05 & .54 & .73 \\
\hline SAVRY historical & $.22 * *$ & $.62 *$ & .05 & .53 & .72 \\
\hline SAVRY social & .15 & $.60^{*}$ & .05 & .51 & .70 \\
\hline SAVRY clinical & $.16^{*}$ & $.62 *$ & .05 & .52 & .72 \\
\hline SAVRY protective ${ }^{\mathrm{a}}$ & $-.29 * *$ & $.67 * * *$ & .05 & .58 & .76 \\
\hline CD symptoms & $.18^{*}$ & $.61^{*}$ & .05 & .51 & .71 \\
\hline
\end{tabular}

Note PCL $\cdot \mathrm{YV}=$ Psychopathy Checklist Youth Version, SAVRY = Structured Assessment of Violence Risk in Youth, $\mathrm{CD}=$ conduct disorder; $\mathrm{AUC}=$ area under the curve, $\mathrm{SE}=$ standard error, $\mathrm{a}=$ for the SAVRY protective score, the prediction was for non-offending

${ }^{*} p<05,{ }^{* *} p<01,{ }^{* * *} p<001$

For the one-year follow-up period, the only significant predictor of non-violent offending was factor 3 (behavioural) with an AUC of .59, $p<.05$, but this is considered to be low in terms of accuracy. The SAVRY risk score was also significant at $p<.05(\mathrm{AUC}=.58)$ and the SAVRY clinical score had an AUC of $.60, p<.05$ but again, this is considered low. All other scores were not significant (see Table 17). 
Table 17

Predictive Accuracy (ROC) and 95\% Confidence Intervals (CI) for Non-Violent Recidivism for One-Year Follow-Up Period

\begin{tabular}{|c|c|c|c|c|c|}
\hline \multirow[t]{2}{*}{ Variable } & \multirow[t]{2}{*}{$r$} & \multirow[t]{2}{*}{ AUC } & \multirow[t]{2}{*}{$S E$} & \multicolumn{2}{|c|}{ Asymptotic $95 \%$ CI } \\
\hline & & & & Lower & Upper \\
\hline PCL:YV 20 item & .08 & .55 & .04 & .48 & .63 \\
\hline PCL:YV 13 item & .07 & .54 & .04 & .46 & .61 \\
\hline PCL:YV 18 item & .09 & .56 & .04 & .48 & .63 \\
\hline Factor 1 - interpersonal & -.04 & .48 & .04 & .40 & .56 \\
\hline Factor 2 - affective & .08 & .56 & .04 & .48 & .63 \\
\hline Factor 3 - behavioural & .15 & $.59^{*}$ & .04 & .51 & .67 \\
\hline Factor 4 - antisocial & .09 & .58 & .04 & .50 & .65 \\
\hline SAVRY risk & .14 & $.58^{*}$ & .04 & .51 & .66 \\
\hline SAVRY historical & .11 & .57 & .04 & .49 & .64 \\
\hline SAVRY social & .07 & .54 & .04 & .46 & .62 \\
\hline SAVRY clinical & .15 & $.60^{*}$ & .04 & .52 & .67 \\
\hline SAVRY protective ${ }^{\mathrm{a}}$ & -.13 & .57 & .04 & .50 & .65 \\
\hline CD symptoms & .11 & .57 & .04 & .50 & .65 \\
\hline
\end{tabular}

Note: PCL:YV = Psychopathy Checklist: Youth Version, SAVRY = Structured Assessment of Violence Risk in Youth, $\mathrm{CD}=$ conduct disorder; $\mathrm{AUC}=$ area under the curve, $\mathrm{SE}=$ standard error; $\mathrm{a}=$ for the SAVRY protective score, the prediction was for non-offending.

${ }^{*} p<.05$

SAVRY risk score was found to be a significant predictor of non-violent recidivism during the 5-year follow-up period $(\mathrm{AUC}=.61, \mathrm{p}<.01)$, as was SAVRY social $(\mathrm{AUC}=.60, \mathrm{p}<$ $.05)$ and SAVRY clinical $(\mathrm{AUC}=.60, \mathrm{p}<.05)$. The number of $\mathrm{CD}$ symptoms was also predictive of non-violent recidivism $(\mathrm{AUC}=.61, \mathrm{p}<.01)$. These were all considered to be in the low range. The protective score was found to be a significant predictor of non-violent desistence during the 5-year follow-up, AUC $=.61, \mathrm{p}<.01$ (see Table 18). 
Table 18

Predictive Accuracy (ROC) and 95\% Confidence Intervals (CI) for Non-Violent Recidivism for Five-Year Follow-Up Period

\begin{tabular}{|c|c|c|c|c|c|}
\hline \multirow[t]{2}{*}{ Variable } & \multirow[t]{2}{*}{$r$} & \multirow[t]{2}{*}{ AUC } & \multirow[t]{2}{*}{$S E$} & \multicolumn{2}{|c|}{ Asymptotic $95 \% \mathrm{CI}$} \\
\hline & & & & Lower & Upper \\
\hline PCL:YV 20 item & .04 & .54 & .04 & .46 & .61 \\
\hline PCL:YV 13 item & .02 & .51 & .04 & .43 & .59 \\
\hline PCL:YV 18 item & .05 & .53 & .04 & .45 & .61 \\
\hline Factor 1 - interpersonal & -.01 & .50 & .04 & .42 & .58 \\
\hline Factor 2 - affective & -.01 & .51 & .04 & .43 & .59 \\
\hline Factor 3 - behavioural & .09 & .55 & .04 & .48 & .63 \\
\hline Factor 4 - antisocial & .08 & .55 & .04 & .47 & .62 \\
\hline SAVRY risk & $.20 * *$ & $.61^{* *}$ & .04 & .53 & .69 \\
\hline SAVRY historical & $.16^{*}$ & .58 & .04 & .50 & .66 \\
\hline SAVRY social & $.17^{*}$ & $.60^{*}$ & .04 & .53 & .68 \\
\hline SAVRY clinical & $.17 * *$ & $.60^{*}$ & .04 & .52 & .68 \\
\hline SAVRY protective ${ }^{\mathrm{a}}$ & $-.17^{*}$ & $.61 * *$ & .04 & .53 & .69 \\
\hline CD symptoms & $.18^{* *}$ & $.61 * *$ & .04 & .53 & .69 \\
\hline
\end{tabular}

Note: PCL:YV = Psychopathy Checklist: Youth Version, SAVRY = Structured Assessment of Violence Risk in Youth, $\mathrm{CD}=$ conduct disorder; $\mathrm{AUC}=$ area under the curve, $\mathrm{SE}=$ standard error; $\mathrm{a}=$ for the SAVRY protective score, the prediction was for non-offending.

${ }^{*} p<.05,{ }^{* *} p<.01$

During the 10-year follow-up period, SAVRY protective was a significant predictor of desistence of non-violent offending $(\mathrm{AUC}=.62, p<.01)$, whereas SAVRY risk $(\mathrm{AUC}=.59, p<$ $.05), \mathrm{SAVRY}$ social $(\mathrm{AUC}=.60, p<.05)$ and number of $\mathrm{CD}$ symptoms $(\mathrm{AUC}=.61, p<.05)$ were found to be predictive of recidivism but all in the low accuracy level (see Table 19). 
Table 19

Predictive Accuracy (ROC) and 95\% Confidence Intervals (CI) for Non-Violent Recidivism for Ten-Year Follow-Up Period

\begin{tabular}{|c|c|c|c|c|c|}
\hline \multirow[t]{2}{*}{ Variable } & \multirow[t]{2}{*}{$r$} & \multirow[t]{2}{*}{$\mathrm{AUC}$} & \multirow[t]{2}{*}{$S E$} & \multicolumn{2}{|c|}{ Asymptotic $95 \% \mathrm{CI}$} \\
\hline & & & & Lower & Upper \\
\hline PCL:YV 20 item & .05 & .54 & .05 & .45 & .63 \\
\hline PCL:YV 13 item & .04 & .52 & .05 & .43 & .61 \\
\hline PCL:YV 18 item & .07 & .54 & .05 & .44 & .63 \\
\hline Factor 1 - interpersonal & -.05 & .48 & .05 & .39 & .57 \\
\hline Factor 2 - affective & -.02 & .50 & .05 & .41 & .59 \\
\hline Factor 3 - behavioural & $.16^{*}$ & .59 & .05 & .50 & .68 \\
\hline Factor $4-$ antisocial & .11 & .57 & .05 & .48 & .66 \\
\hline SAVRY risk & $.17^{*}$ & $.59^{*}$ & .05 & .50 & .68 \\
\hline SAVRY historical & $.15^{*}$ & .57 & .05 & .48 & .67 \\
\hline SAVRY social & $.15^{*}$ & $.60 *$ & .05 & .51 & .69 \\
\hline SAVRY clinical & .11 & .57 & .05 & .48 & .67 \\
\hline SAVRY protective ${ }^{\mathrm{a}}$ & $-.22 * *$ & $.62 * *$ & .05 & .53 & .71 \\
\hline CD symptoms & $.19 * *$ & $.61^{*}$ & .05 & .52 & .71 \\
\hline
\end{tabular}

Note: PCL:YV = Psychopathy Checklist: Youth Version, SAVRY = Structured Assessment of Violence Risk in Youth, $\mathrm{CD}=$ conduct disorder; $\mathrm{AUC}=$ area under the curve, $\mathrm{SE}=$ standard error; $\mathrm{a}=$ for the SAVRY protective score, the prediction was for non-offending.

${ }^{*} p<.05,{ }^{* *} p<.01$

For violent prediction within the first year, the PCL:YV showed moderate ability to predict violent offending $(\mathrm{AUC}=.68, p<.01)$ with the 18 -item model showing better accuracy in the prediction of violent offending, $\mathrm{AUC}=.70, p<.001$. The interpersonal factor (AUC $=$ $.63)$, behavioural factor $(\mathrm{AUC}=.68)$, and the antisocial factor $(\mathrm{AUC}=.69)$ all showed predictive accuracy at the $p<.001$ level and all are considered to be in the moderate range. The SAVRY 
risk score was not predictive of violent offending within the first year $(\mathrm{AUC}=.62)$ and only the clinical rating $(\mathrm{AUC}=.67)$ showed any predictive power (see Table 20 ).

Table 20

Predictive Accuracy (ROC) and 95\% Confidence Intervals (CI) for Violent Recidivism for OneYear Follow-Up Period

\begin{tabular}{|c|c|c|c|c|c|}
\hline \multirow[t]{2}{*}{ Variable } & \multirow[t]{2}{*}{$r$} & \multirow[t]{2}{*}{ AUC } & \multirow[t]{2}{*}{$S E$} & \multicolumn{2}{|c|}{ Asymptotic 95\% CI } \\
\hline & & & & Lower & Upper \\
\hline PCL:YV 20 item & $.20 * *$ & $.68 * *$ & .04 & .59 & .76 \\
\hline PCL:YV 13 item & $.18^{* *}$ & $.67 * *$ & .05 & .58 & .76 \\
\hline PCL:YV 18 item & $.21 * *$ & $.70 * * *$ & .04 & .61 & .78 \\
\hline Factor 1 - interpersonal & $.13 *$ & $.63^{*}$ & .06 & .52 & .74 \\
\hline Factor $2-$ affective & $.19 * *$ & $.68 * *$ & .05 & .58 & .77 \\
\hline Factor 3 - behavioural & .11 & .58 & .05 & .48 & .68 \\
\hline Factor $4-$ antisocial & $.20 * *$ & $.69 * *$ & .05 & .59 & .78 \\
\hline SAVRY risk & .12 & .62 & .05 & .52 & .71 \\
\hline SAVRY historical & .08 & .58 & .05 & .49 & .69 \\
\hline SAVRY social & .00 & .51 & .06 & .40 & .62 \\
\hline SAVRY clinical & $.18 * *$ & $.67 * *$ & .05 & .58 & .77 \\
\hline SAVRY protective ${ }^{a}$ & -.03 & .51 & 06 & .39 & .63 \\
\hline CD symptoms & .10 & .61 & .06 & .49 & .73 \\
\hline
\end{tabular}

The predictive accuracy of the PCL:YV decreased for 5-year prediction of violent offending to an AUC of .66 (20-item model), which was the highest of all the total score models. The factor scores were all in the low range of accuracy (AUCs ranging from .54 to .62). SAVRY 
risk was slightly less predictive than the PCL:YV total scores $(\mathrm{AUC}=.62)$ for the 5-year period, which was significant at the $p<.01$ level, but is considered to be low. Only the clinical subscale was significant at the $p<.001$ level, but with an AUC of .64, this is still considered to be in the low-moderate range (see Table 21).

Table 21

Predictive Accuracy (ROC) and 95\% Confidence Intervals (CI) for Violent Recidivism for FiveYear Follow-Up Period

\begin{tabular}{|c|c|c|c|c|c|}
\hline \multirow[t]{2}{*}{ Variable } & \multirow[t]{2}{*}{$r$} & \multirow[t]{2}{*}{ AUC } & \multirow[t]{2}{*}{$S E$} & \multicolumn{2}{|c|}{ Asymptotic 95\% CI } \\
\hline & & & & Lower & Upper \\
\hline PCL:YV 20 item & $.16^{*}$ & $.66^{*}$ & .04 & .52 & .67 \\
\hline PCL:YV 13 item & .11 & .57 & .04 & .49 & .64 \\
\hline PCL:YV 18 item & $.14^{*}$ & $.59^{*}$ & .04 & .52 & .66 \\
\hline Factor 1 - interpersonal & .09 & .56 & .04 & .48 & .63 \\
\hline Factor $2-$ affective & .05 & .54 & .04 & .46 & .62 \\
\hline Factor 3 - behavioural & $.15^{*}$ & $.58^{*}$ & .04 & .50 & .65 \\
\hline Factor $4-$ antisocial & $.19^{* *}$ & $.62 * *$ & .04 & .54 & .69 \\
\hline SAVRY risk & $.21^{* *}$ & $.62 * *$ & .04 & .55 & .69 \\
\hline SAVRY historical & $.18^{* *}$ & $.60^{* *}$ & .04 & .53 & .68 \\
\hline SAVRY social & .08 & .55 & .04 & .48 & .62 \\
\hline SAVRY clinical & $.24 * *$ & $.64 * * *$ & .04 & .57 & .71 \\
\hline SAVRY protective ${ }^{\mathrm{a}}$ & -.11 & .56 & .04 & .48 & .63 \\
\hline CD symptoms & $.13^{*}$ & $.58^{*}$ & .04 & 51 & .66 \\
\hline
\end{tabular}

Lastly, for 10-year predictive accuracy, the PCL:YV total scores (all models) and factor scores did not predict violent offending (AUCs of .49 to .63). The SAVRY risk score was 
significant at $p<.001$ level with an AUC of .67 and the historical rating was also predictive of violent offending within 10 years with an AUC of .67, $p<.001$ (see Table 22).

\section{Table 22}

Predictive Accuracy (ROC) and 95\% Confidence Intervals (CI) for Violent Recidivism for TenYear Follow-Up Period

\begin{tabular}{|c|c|c|c|c|c|}
\hline \multirow[t]{2}{*}{ Variable } & \multirow[t]{2}{*}{$r$} & \multirow[t]{2}{*}{ AUC } & \multirow[t]{2}{*}{$S E$} & \multicolumn{2}{|c|}{ Asymptotic 95\% CI } \\
\hline & & & & Lower & Upper \\
\hline PCL:YV 20 item & .09 & .57 & .04 & .49 & .66 \\
\hline PCL:YV 13 item & .05 & .53 & .04 & .45 & .62 \\
\hline PCL:YV 18 item & .10 & .57 & .04 & .48 & .65 \\
\hline Factor 1 - interpersonal & -.04 & .49 & .04 & .40 & .57 \\
\hline Factor 2 - affective & -.01 & .51 & .04 & .42 & .60 \\
\hline Factor 3 - behavioural & $.17^{*}$ & $.60^{*}$ & .04 & .52 & .69 \\
\hline Factor 4 - antisocial & $.21 * *$ & $.63 * *$ & .04 & .54 & .71 \\
\hline SAVRY risk & $.32 * *$ & $.67 * * *$ & .04 & .59 & .75 \\
\hline SAVRY historical & $.31 * *$ & $.67 * * *$ & .04 & .59 & .75 \\
\hline SAVRY social & $.20 * *$ & $.62 *$ & .04 & .54 & .70 \\
\hline SAVRY clinical & $.25^{* *}$ & $.65^{*}$ & .04 & .56 & .73 \\
\hline SAVRY protective ${ }^{\mathrm{a}}$ & $-.23 * *$ & $.63^{* *}$ & .04 & .55 & .71 \\
\hline CD symptoms & $.21 * *$ & $.62 * *$ & .04 & .53 & .70 \\
\hline
\end{tabular}

Survival Analyses. The ability to predict a re-offence while accounting for the time at risk is best performed using Cox regressions. This analytic tool is a semi-parametric test that examines the association between a predictor variable and an event (re-offence) accounting for the time in which this event can occur. The dependent variable - time at risk - therefore is 
dependent upon the proportion of cases surviving. In this study, that means those who have made it to the end of the study period without having been convicted of a new offence. Time at risk is calculated for non-violent and violent offences based on the number of days between release from custody and the first conviction for each group of offences within 1-year, 5-years and 10years. For those with no recidivism data, time at risk is calculated until the end of the follow-up period.

In examining the results of the Cox regression models, it is important to examine the regression coefficients. Predictors with positive coefficients (B) decrease survival time (reoffence occurs more quickly after release) whereas negative coefficients imply that offences occur later after release. Further, Exp [B] (hazard functions) is often used to give an indication of the likelihood of an event. In this study, for example, an Exp [B] of 1.7 indicates that one unit increase in the total score would yield a $70 \%$ increase in the likelihood that a new offence would occur.

To achieve this end, several models were computed. Models were computed for nonviolent and violent recidivism and not for general recidivism given that the ROC analysis failed to reveal any risk factor was related to subsequent offending. Non-violent recidivism was kept as an outcome variable to discern whether survival analysis could predict differences on this outcome based on level of risk as per the PCL:YV and the SAVRY. All models were computed with the total score entered first and then a second model was examined with only the factor scores entered. Firstly, non-violent and violent recidivism was examined during 1-year post release, 5-years post release, and 10-years post release. For offending within the first year, the results indicate that the PCL:YV total score was significantly related to the time until first nonviolent offence $(-2 \log$ likelihood $=535.76), \chi^{2}(1)=4.04, p<.04$. With a positive beta 
coefficient, the results indicate that having a higher score on the PCL:YV was related to youth re-offending more quickly than those scoring low. Further, with every unit increase in PCL:YV score, youth had a $4 \%$ greater risk of non-violent recidivism. None of the PCL:YV factor scores were related to the imminence of non-violent offending. SAVRY risk was also found to be significantly related to the time until first non-violent offence within the first year following release, $(-2 \log$ likelihood $=535.45), \chi^{2}(1)=4.45, p<.03$ with higher risk scores related to the imminence of non-violent offending. With every unit increase in SAVRY risk score, youth had a $4 \%$ increase in risk in non-violent offending. None of the subscales however were related to the time until first non-violent offence.

When violent offending was examined within the first year, the PCL:YV total score (20item) was not related to the time until first violent offence, $(-2 \log$ likelihood $=102.04), \chi^{2}(1)=$ $3.32, p=.07$. The only significant PCL:YV factor relating to the time until first violent offence within the first year following release was the antisocial factor (factor 4), indicating that higher scores were related to offenders recidivating more quickly following release, and that with every unit increase in the factor score, the offender had a $88 \%$ increase in risk in offending violently. The SAVRY risk was not related to time until first violent offence, $(-2 \log$ likelihood $=105.35)$, $\chi^{2}(1)=.41, p=.52$, nor were any of the SAVRY subscales (see Table 23). 
Table 23

Cox Regression - One Year Follow-Up

Predictor

$$
B \quad S E \quad \text { Wald } d f \quad p \quad \operatorname{Exp}[B] \frac{\operatorname{Exp}[B] 95 \% C I}{\text { Lower upper }}
$$

Non-Violent Offending

Model 1:

PCL:YV total

Model 2:

Factor 1 - interpersonal

Factor 2 - affective

Factor 3 - behavioural

Factor 4 - antisocial
.41

.02

4.01

1

.04

1.04

1.00

1.08

$\begin{array}{rr}-.07 \quad .07 \\ .03 & .08\end{array}$

$.03 \quad .08$

$1.21 \quad 1$

$.14 \quad 1$

$.16 \quad .09$

3.45

.10

.08

1.42

1

.27

.71

.93

1.03

.82

1.06

.06

1.18

.88

1.22

1.41

.23

1.10

.99

1.29

Model 1:

SAVRY risk

Model 2:

SAVRY historical

SAVRY social

SAVRY clinical

SAVRY protective

$\begin{array}{cccccccc}.04 & .02 & 4.44 & 1 & .04 & 1.04 & 1.00 & 1.08 \\ & & & & & & & \\ .08 & .05 & 2.38 & 1 & .12 & 1.08 & .98 & 1.19 \\ .06 & .08 & .54 & 1 & .46 & .94 & .80 & 1.11 \\ .03 & .05 & .37 & 1 & .54 & 1.03 & .93 & 1.14 \\ -.09 & .16 & .30 & 1 & .57 & .92 & .67 & 1.26\end{array}$

\section{Violent Offending}

Model 1:

PCL:YV total

Model 2:

Factor 1 - interpersonal

Factor 2 - affective

Factor 3-behavioural

$.09 \quad .05$

3.20

$1 \quad .07$

1.09

.99

1.21

Factor 4 - antisocial

$\begin{array}{cc}-.02 & .16 \\ .26 & .21 \\ -.20 & .21 \\ .63 & .29\end{array}$

$\begin{array}{lll}.01 & 1 & .92\end{array}$

.98

.72

1.35

$\begin{array}{lll}1.59 & 1 & .21\end{array}$

1.30

.86

1.97

$\begin{array}{lll}.91 & 1 & .34\end{array}$

.82

.55

1.23

$\begin{array}{lll}4.84 & 1 & .03\end{array}$

1.88

1.07

3.28

Model 1:

SAVRY risk

$.03 \quad .04$

$\begin{array}{lll}.40 & 1 & .53\end{array}$

1.03

.95

1.11

Model 2:

SAVRY historical

SAVRY social

SAVRY clinical

SAVRY protective

$\begin{array}{cc}.19 & .12 \\ -.30 & .20 \\ .08 & .11 \\ .24 & .36\end{array}$

$\begin{array}{ccc}2.63 & 1 & .11 \\ 2.14 & 1 & .14 \\ .54 & 1 & .46 \\ .46 & 1 & .50\end{array}$

1.21

.96

1.53

$.74 \quad .50$

1.11

$\begin{array}{ll}1.08 & .87\end{array}$

1.36

1.27

.63

2.57

Note: PCL:YV = Psychopathy Checklist: Youth Version, SAVRY = Structured Assessment of Violence Risk in Youth, $\mathrm{CD}=$ conduct disorder; $\mathrm{SE}=$ standard error, $\mathrm{df}=$ degrees of freedom. 
For non-violent recidivism within the 5-year follow-up period, the PCL:YV was not related to the timing of the first offence, $(-2 \log$ likelihood $=917.14), \chi^{2}(1)=2.33, p=.13$, nor were any of the PCL:YV subscales. Similar results were observed for the SAVRY risk total, (-2 $\log$ likelihood $=917.11), \chi^{2}(1)=2.38, p=.12$, and each of the SAVRY subscales. For this period, no measure was related to the timing of non-violent offending. When violent offending was examined, the PCL:YV total score was significantly related to the imminence of first conviction during the 5-year period, $(-2 \log$ likelihood $=477.72), \chi^{2}(1)=6.62, p<.01$, indicating that having a higher score on the PCL:YV was related to re-offending violently more quickly and that with every unit increase in PCL:YV score yielded a $6 \%$ increase in risk of violent recidivism within 5-years post release. Further, the antisocial factor (factor 4) was found to be related to the time of first violent offence, $\mathrm{B}=.16$, Wald $=3.75, p<.05, \operatorname{Exp}[\mathrm{B}]=1.17$, revealing that having a higher factor 4 score was related to having recidivated violently sooner post release and that with every unit increase of the factor score yielded a $17 \%$ increase in risk of violent offending within 5-years. SAVRY risk was also significantly related to timing of first offence, with higher scores associated with offending occurring more quickly after release, $(-2$ $\log$ likelihood $=479.35), \chi^{2}(1)=5.33, p=.02$. With every unit increase in SAVRY score, there was a $5 \%$ increase in the likelihood that an offender would recidivate violently within 5 years. The only SAVRY subscale that was related to the time until first offence was the SAVRY historical subscale with this factor related to the imminence of violent offending and with every unit increase yielding a $11 \%$ increase in risk of violent offending within 5-years (see Table 24). 
Table 24

Cox Regression - Five Year Follow-Up

Predictor $\quad B \quad S E \quad$ Wald $d f \quad p \quad \operatorname{Exp}[B] \frac{\operatorname{Exp}[B] 95 \% C I}{\text { Lower upper }}$

Non-Violent Offending

Model 1:

PCL:YV total

Model 2:

Factor 1 - interpersonal

Factor 2 - affective

Factor 3 - behavioural

Factor 4 - antisocial

$\begin{array}{cccccccc}.22 & .02 & 2.32 & 1 & .13 & 1.02 & .99 & 1.05 \\ & & & & & & & \\ -.01 & .05 & .01 & 1 & .91 & 1.00 & .91 & 1.08 \\ -.07 & .06 & 1.33 & 1 & .25 & .93 & .83 & 1.05 \\ .09 & .07 & 2.08 & 1 & .15 & 1.01 & .97 & 1.25 \\ .08 & .06 & 1.94 & 1 & .16 & 1.09 & .97 & 1.23 \\ & & & & & & & \\ .02 & .01 & 2.38 & 1 & .12 & 1.02 & .99 & 1.05 \\ & & & & & & & \\ -.01 & .04 & .03 & 1 & .88 & .99 & .93 & 1.07 \\ -.01 & .06 & .05 & 1 & .83 & .99 & .88 & 1.10 \\ .04 & .04 & 1.08 & 1 & .30 & 1.04 & .97 & 1.12 \\ -.13 & .12 & 1.13 & 1 & .29 & .88 & .70 & 1.11\end{array}$

Model 1:

SAVRY risk

Model 2:

SAVRY historical

SAVRY social

SAVRY clinical

SAVRY protective

$-.13 \quad .12$

Violent Offending

Model 1:

PCL:YV total

Model 2:

Factor 1 - interpersonal

Factor 2 - affective

Factor 3 - behavioural

$-.02$

$.05 \quad .02$

$\begin{array}{lll}6.53 & 1 & .01\end{array}$

1.06

1.01

1.10

Factor 4 - antisocial

.11

.07

.05

1

$.82 \quad 1.02$

.89

1.17

.11

.09

.06

.81

.98

.83

1.16

.16

1.62

1.12

.94

1.34

.08

$\begin{array}{lll}3.75 & 1 & .05\end{array}$

1.17

1.00

1.38

Model 1:

SAVRY risk

$.04 \quad .02$

$\begin{array}{lll}5.27 & 1 & .02\end{array}$

1.05

1.01

1.09

Model 2:

SAVRY historical

SAVRY social

$.11 \quad .05$

$\begin{array}{lll}4.60 & 1 & .03\end{array}$

$\begin{array}{ll}1.11 & 1.01\end{array}$

1.22

$\begin{array}{lllll}.003 & .09 & .001 & 1 & .97\end{array}$

1.00

.85

1.19

SAVRY clinical

$.02 \quad .05$

$\begin{array}{lll}.18 & 1 & .67\end{array}$

1.02

.93

1.13

SAVRY protective

$.17 \quad .17$

$\begin{array}{lll}.95 & 1 & .33\end{array}$

1.19

.84

1.66

Note: PCL:YV = Psychopathy Checklist: Youth Version, SAVRY = Structured Assessment of Violence Risk in Youth, $\mathrm{CD}=$ conduct disorder; $\mathrm{SE}=$ standard error, $\mathrm{df}=$ degrees of freedom. 
No measures were found to be associated with timing of first non-violent or violent offence during the 10 year follow-up period (see Table 25). 
Table 25

Cox Regression - Ten Year Follow-Up

Predictor $\quad B \quad S E$ Wald $d f \quad p \quad \operatorname{Exp}[B] \frac{\operatorname{Exp}[B] 95 \% C I}{\text { Lower upper }}$

Non-Violent Offending

Model 1:

PCL:YV total

Model 2:

Factor 1 - interpersonal

Factor 2 - affective

Factor 3 - behavioural

Factor 4 - antisocial

$\begin{array}{cccccccc}.02 & .02 & .99 & 1 & .32 & 1.02 & .99 & 1.05 \\ -.01 & .06 & .003 & 1 & .96 & 1.00 & .89 & 1.11 \\ .01 & .07 & .01 & 1 & .92 & 1.01 & .87 & 1.16 \\ .01 & .08 & .01 & 1 & .95 & 1.01 & .87 & 1.16 \\ .08 & .07 & 1.15 & 1 & .28 & 1.08 & .94 & 1.24\end{array}$

Model 1:

SAVRY risk

Model 2:

SAVRY historical

SAVRY social

SAVRY clinical

SAVRY protective

$\begin{array}{cccccccc}.01 & .02 & .11 & 1 & .74 & 1.01 & .97 & 1.04 \\ .05 & .04 & 1.52 & 1 & .22 & 1.05 & .97 & 1.15 \\ -.05 & .06 & .66 & 1 & .42 & .95 & .84 & 1.08 \\ -.04 & .04 & .75 & 1 & .39 & .97 & .89 & 1.04 \\ -.11 & .14 & .59 & 1 & .44 & .90 & .69 & 1.18\end{array}$

Violent Offending

Model 1:

PCL:YV total

.03

.02

$2.01 \quad 1 \quad .16$

1.03

.99

1.07

Model 2:

Factor 1 - interpersonal

Factor 2 - affective

$-.02 \quad .09$

$-.07 \quad .10$

$\begin{array}{lll}.03 & 1 & .86\end{array}$

.99

.83

1.17

Factor 3 - behavioural

$.16 \quad .09$

$.44 \quad 1 \quad .51$

.94

.77

1.14

Factor 4 - antisocial

$.04 \quad .08$

$\begin{array}{lll}2.90 & 1 & .09\end{array}$

1.17

1.41

$\begin{array}{lll}.26 & 1 & .61\end{array}$

1.04

.98

1.23

Model 1:

SAVRY risk

.03

.02

$\begin{array}{lll}2.71 \quad 1 & .10\end{array}$

1.03

.99

1.07

Model 2:

SAVRY historical

SAVRY social

$\begin{array}{cccccccc}.09 & .05 & 3.31 & 1 & .07 & 1.10 & .99 & 1.22 \\ .01 & .09 & .01 & 1 & .92 & 1.01 & .84 & 1.22 \\ . .003 & .05 & .003 & 1 & .96 & .98 & .90 & 1.10 \\ .16 & .21 & .59 & 1 & .44 & 1.17 & .78 & 1.77\end{array}$

SAVRY clinical

SAVRY protective

Note: PCL:YV = Psychopathy Checklist: Youth Version, SAVRY = Structured Assessment of Violence Risk in Youth, $\mathrm{CD}=$ conduct disorder; $\mathrm{SE}=$ standard error, $\mathrm{df}=$ degrees of freedom. 
Survival analyses utilizing Kaplan-Meier curves were undertaken to examine if group differences based on the PCL:YV and SAVRY total scores would be noted (see Table 26 for mean survival times). These tests indicated if the proportion of participants that remained free of

Table 26

Mean Survival Times for PCL:YV and SAVRY groups

Variable One Year Five Years Ten Years

$\begin{array}{lccc}\text { PCL:YV Groups } & & & \\ \text { Low } & 300.2(14.8) & 930.9(46.2) & 1637.1(269.5) \\ \text { Medium } & 293.9(11.3) & 959.9(52.9) & 1707.4(118.6) \\ \text { High } & 271.7(13.9) & 861.5(50.2) & 1459.6(180.9) \\ & & & \\ \text { SAVRY Groups } & 318.7(15.8) & 1166.4(48.8) & 2286.0(307.9) \\ \quad \text { Low } & 283.9(9.5) & 896.3(51.5) & 1534.2(114.6) \\ \text { Medium } & 273.5(19.7) & 759.8(105.3) & 1083.8(255.4) \\ \text { High } & & \text { Violent Offending } \\ & & \end{array}$

$\begin{array}{llll}\text { PCL:YV Groups } & & & \\ \text { Low } & 359.6(5.4) & 1550.7(61.3) & 2597.2(218.9) \\ \text { Medium } & 344.3(6.6) & 1433.2(61.8) & 2566.7(148.8) \\ \text { High } & 335.2(8.0) & 1233.5(72.8) & 2035.0(168.2)\end{array}$

$\begin{array}{lccc}\text { SAVRY Groups } & & & \\ \text { Low }^{\mathrm{a}} & 365.0(0) & 1646.4(66.1) & 3052.8(208.6) \\ \text { Medium } & 339.0(71.6) & 1352.0(41.5) & 2293.2(124.5) \\ \text { High } & 350.9(51.4) & 1318.7(94.6) & 1908.7(231.1)\end{array}$

Note: standard error is provided in parenthesis; $\mathrm{a}=$ no participants who scored in the low SAVRY group recidivated violently during the first year.

offences (survived), as a function of time free, differed across low, moderate and high risk groups. Kaplan-Meier graphs were computed for non-violent and violent offending for each of 
the three follow-up periods ( 1 year, 5 years, and 10 years; see Figures 3 through 14) for PCL:YV and SAVRY risk groups. Several significant results were obtained. PCL:YV groups were found to have significant $\log$ rank test of equality, $\chi^{2}(2, N=235)=8.06, p<.02$ for violent offending during the first year post release. Pairwise comparisons indicated that the high scoring group ( $M$ $=335.2, S E=8.0)$ significantly differed from the low scoring group $(M=359.6, S E=5.4)$, meaning the high scoring group violently recidivated earlier following release from custody. The moderate scoring group did not significantly differ from the high or low scoring group. The PCL:YV groups also differed on violent offending during the first five years, $\chi^{2}(2, N=235)=$ $7.90, p<.02$. This finding illustrates that the high scoring PCL:YV group recidivated more quickly $(M=1233.5, S E=72.8)$ than youth who scored in the low group $(M=1550.7, S E=$ 61.3). Youth who scored in the moderate range did not recidivate differently than those in the low or high scoring groups. No other comparisons across outcomes were significant for the PCL:YV groups.

When SAVRY risk groups were examined for differences in mean survival time, the log rank test of equality indicated that youth who scored in the high SAVRY risk group recidivated non-violently more quickly than those in the low scoring group during the 5-year follow-up period, $\chi^{2}(2, N=234)=7.87, p<.02\left(M_{\mathrm{hlgh}}=759.8, S E_{\mathrm{hlgh}}=105.3 ; M_{\mathrm{low}}=1166.4, S E_{\mathrm{low}}=\right.$ 48.8). Similarly, the same groups differed on the timing of non-violent offences during the 10year follow-up period, $\chi^{2}(2, N=174)=6.69, p<.04$, indicating again that those in the high scoring group recidivated sooner $(M=1083.8, S E=255.4)$ than youth in the low scoring group $(M=2286.0, S E=307.9)$. The youth also differed on violent recidivism during the five year period, $\chi^{2}(2, N=234)=8.79, p<.02$ and the 10 -year follow-up period, $\chi^{2}(2, N=174)=12.79$, $p<.002$. In these two comparisons, the moderate scoring group recidivated more quickly $\left(\mathrm{M}_{5 y e a r}\right.$ 
$\left.=1318.7, \mathrm{SE}_{5 \text { year }}=94.6 ; \mathrm{M}_{10 \text { year }}=1908.7, \mathrm{SD}_{10 \mathrm{year}}=231.1\right)$ than those in the low scoring group $\left(\mathrm{M}_{5 \text { year }}=1646.4, \mathrm{SD}_{5 \mathrm{year}}=66.1 ; \mathrm{M}_{10 \mathrm{year}}=3052.8, \mathrm{SD}_{10 \mathrm{year}}=208.6\right)$. All other comparisons were not significant.

Figure 3.

Kaplan-Meier Graph-One Year Non-Violent Recidivism for PCL:YV Groups

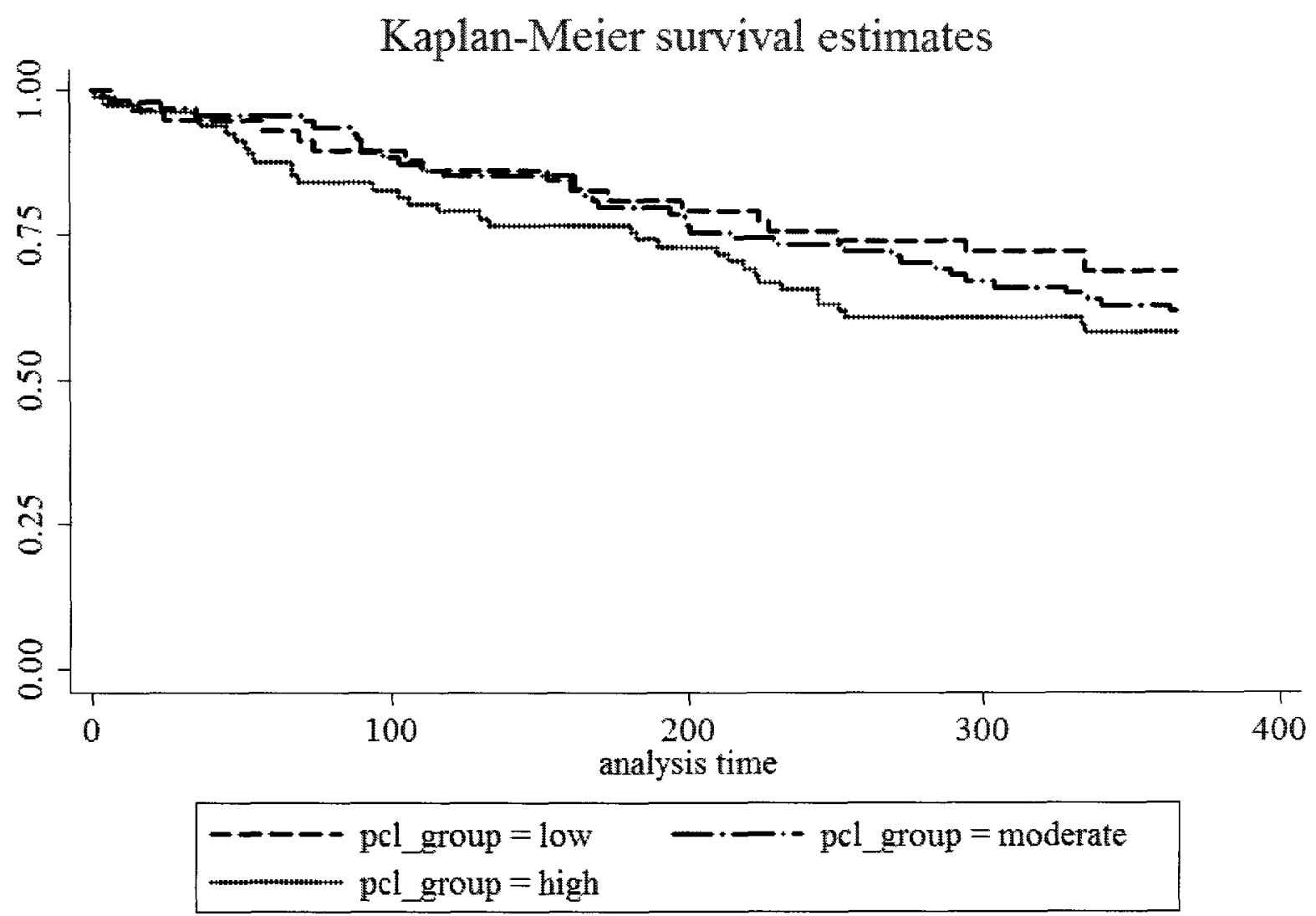


Figure 4.

Kaplan-Meier Graph - Five Year Non-Violent Recidivism for PCL:YV Groups

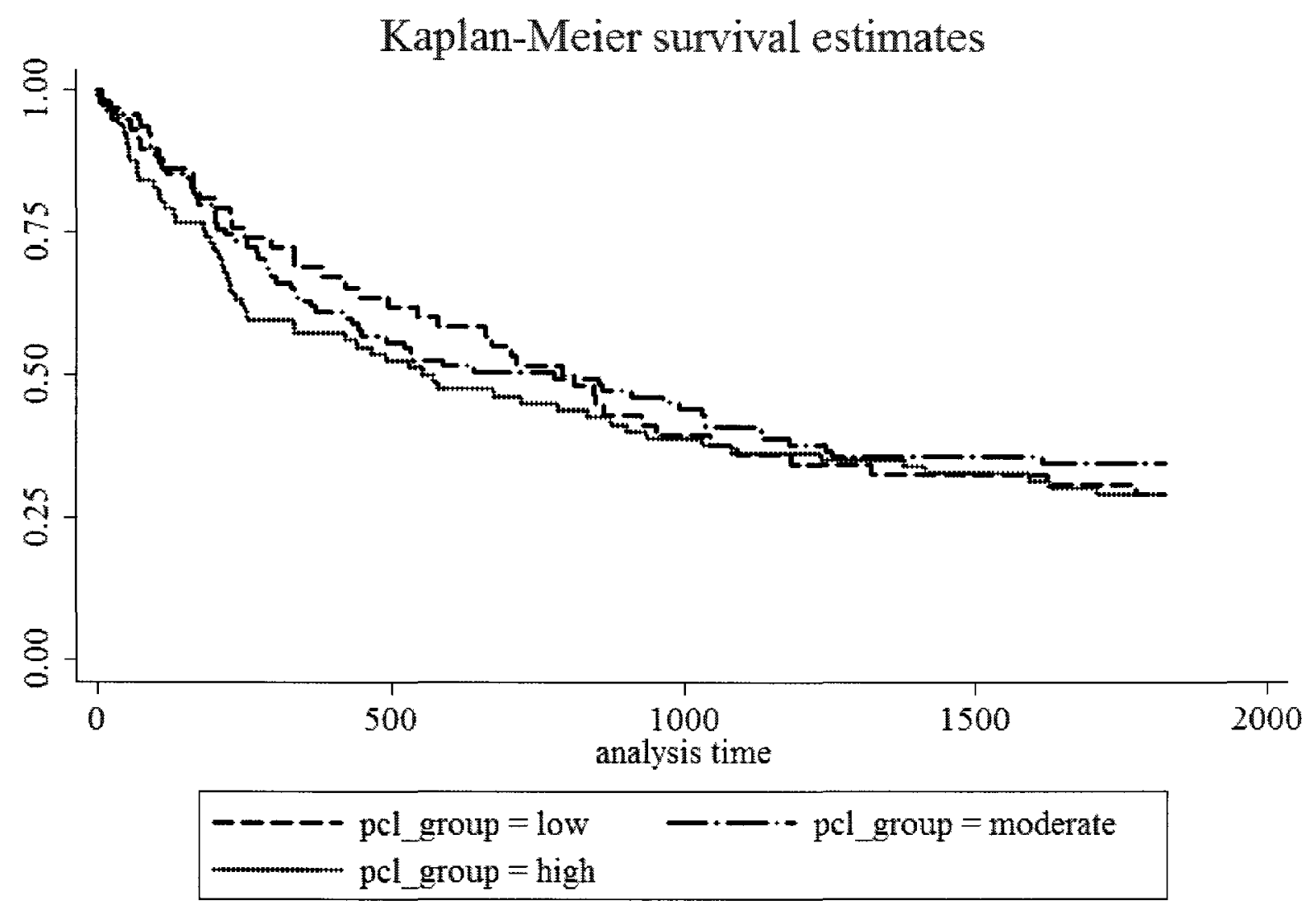


Figure 5.

Kaplan-Meier Graph - Ten Year Non-Violent Recidivism for PCL:YV Groups

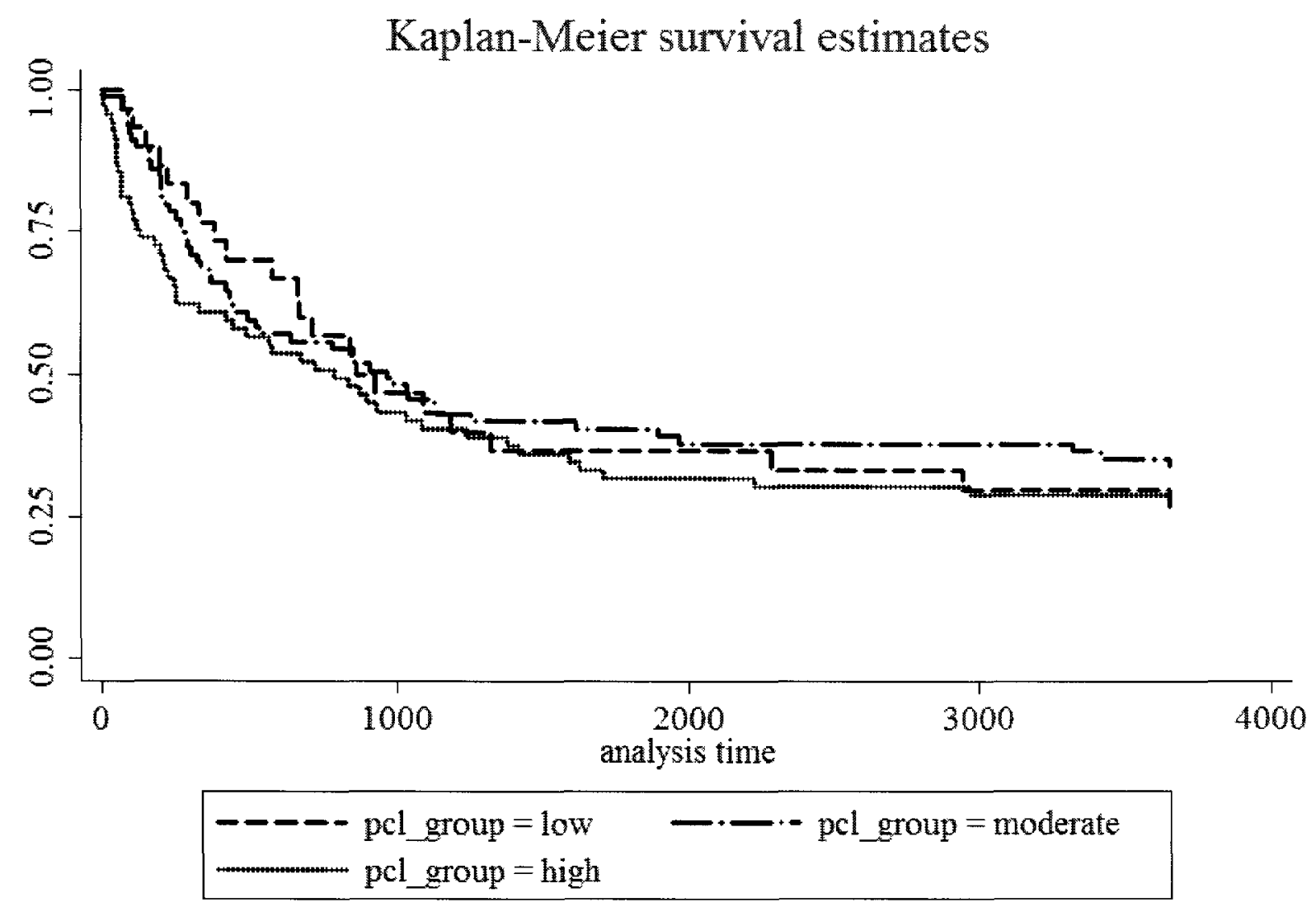


Figure 6.

Kaplan-Meier Graph-One Year Non-Violent Recidivism for SAVRY Groups

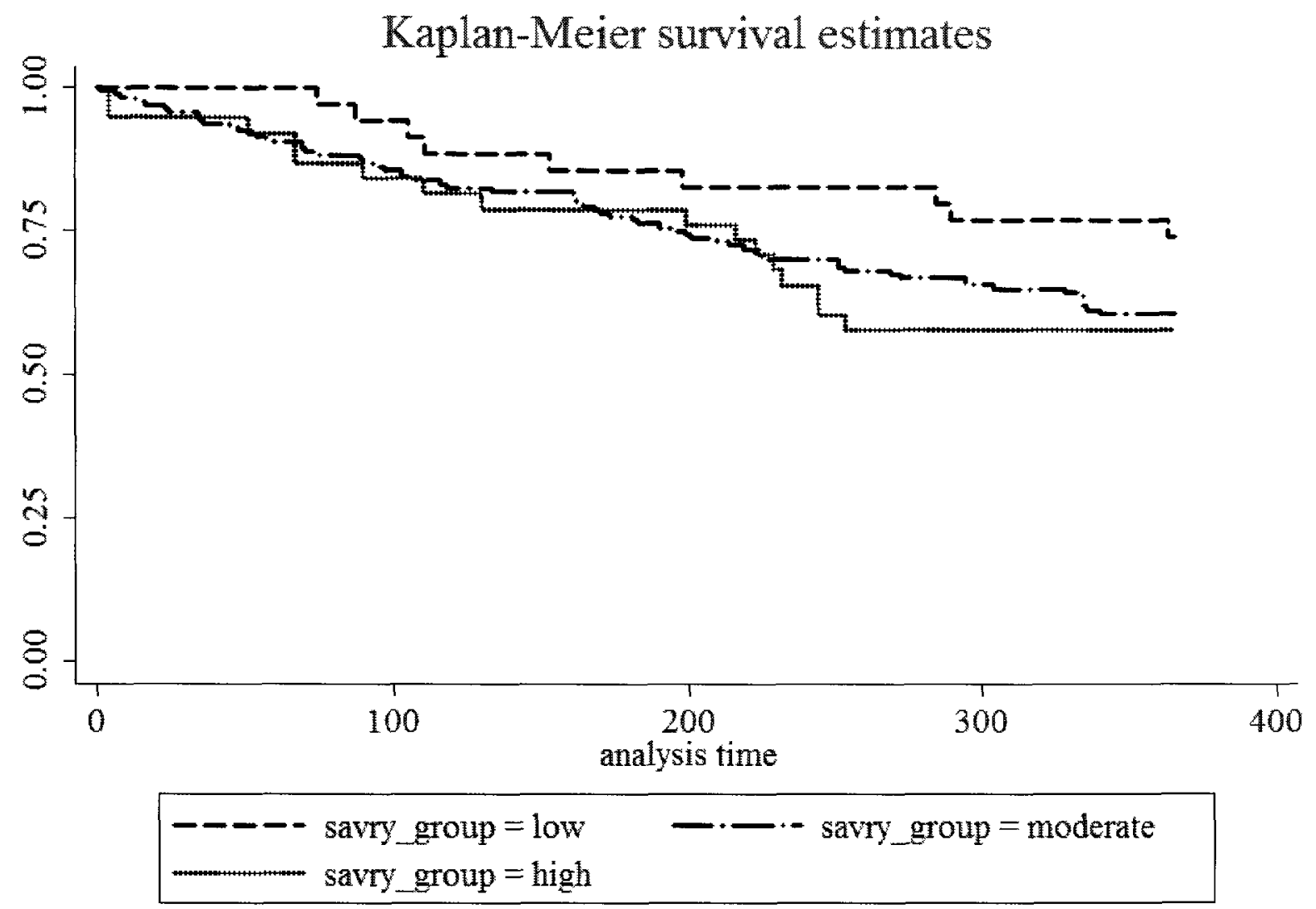


Figure 7.

Kaplan-Meier Graph - Five Year Non-Violent Recidivism for SAVRY Groups

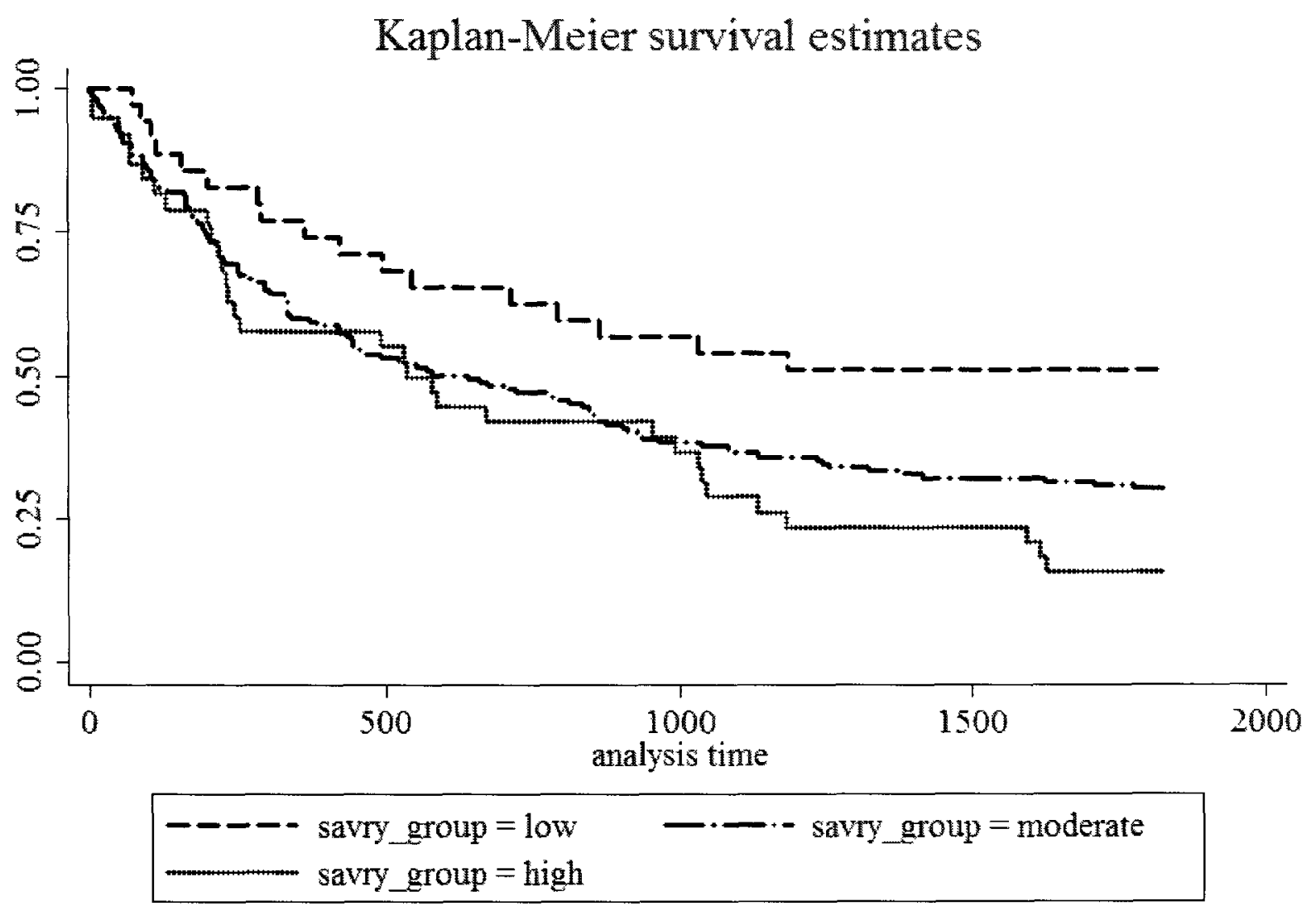


Figure 8.

Kaplan-Meier Graph - Ten Year Non-Violent Recidivism for SAVRY Groups

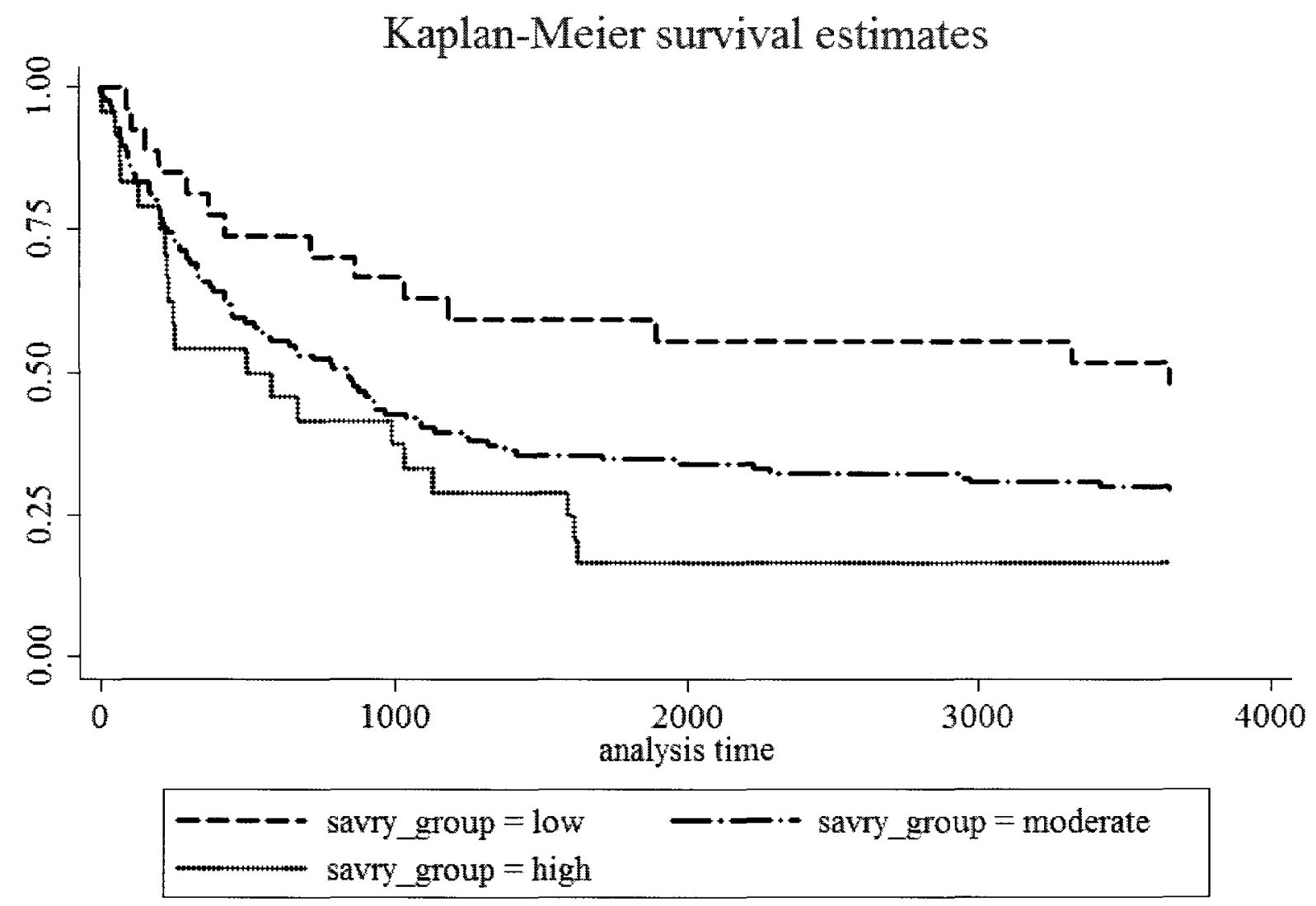


Figure 9.

Kaplan-Meier Graph - One Year Violent Recidivism for PCL:YV Groups

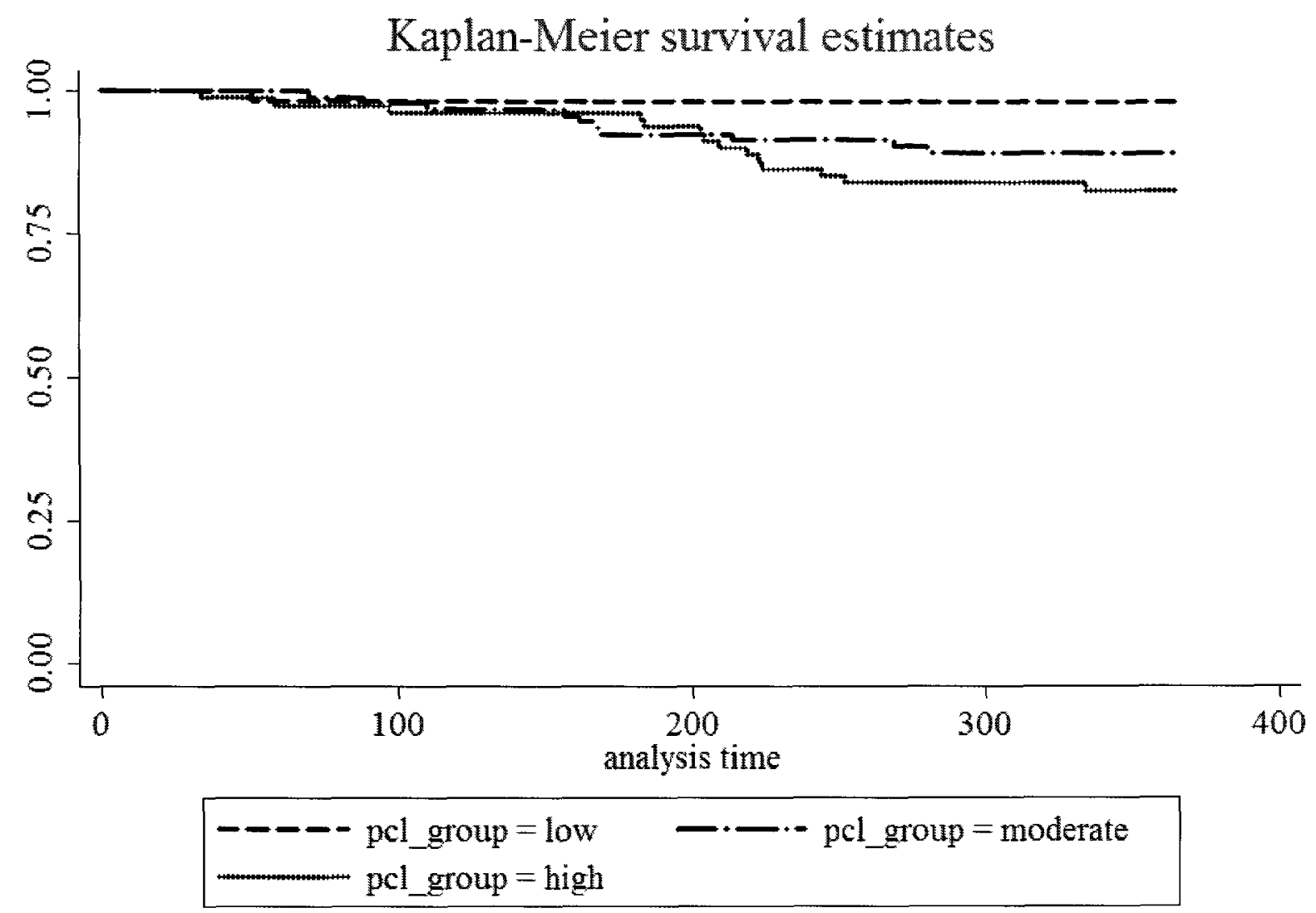


Figure 10.

Kaplan-Meier Graph - Five Year Violent Recidivism for PCL:YV Groups

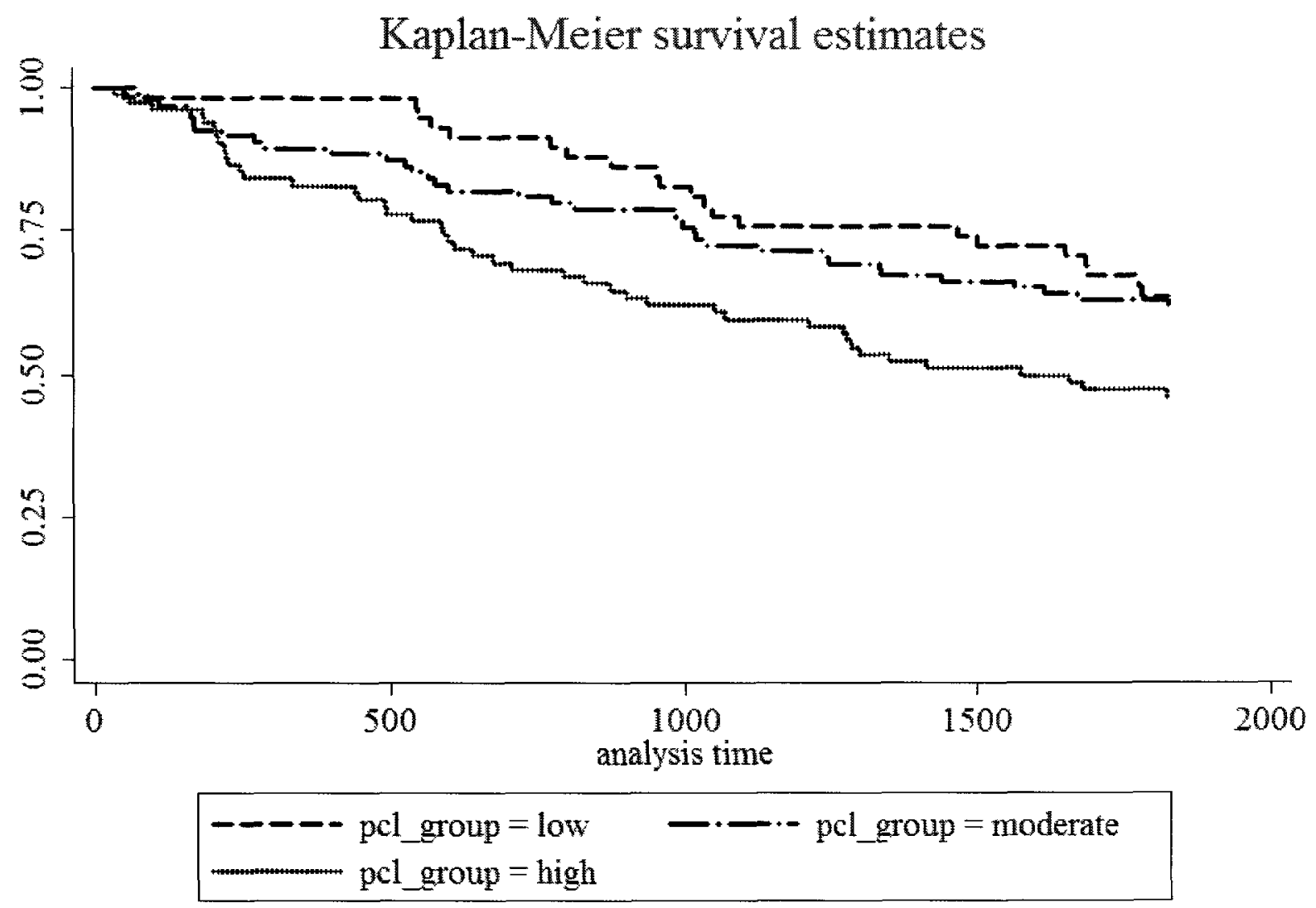


Figure 11.

Kaplan-Meier Graph - Ten Year Violent Recidivism for PCL:YV Groups

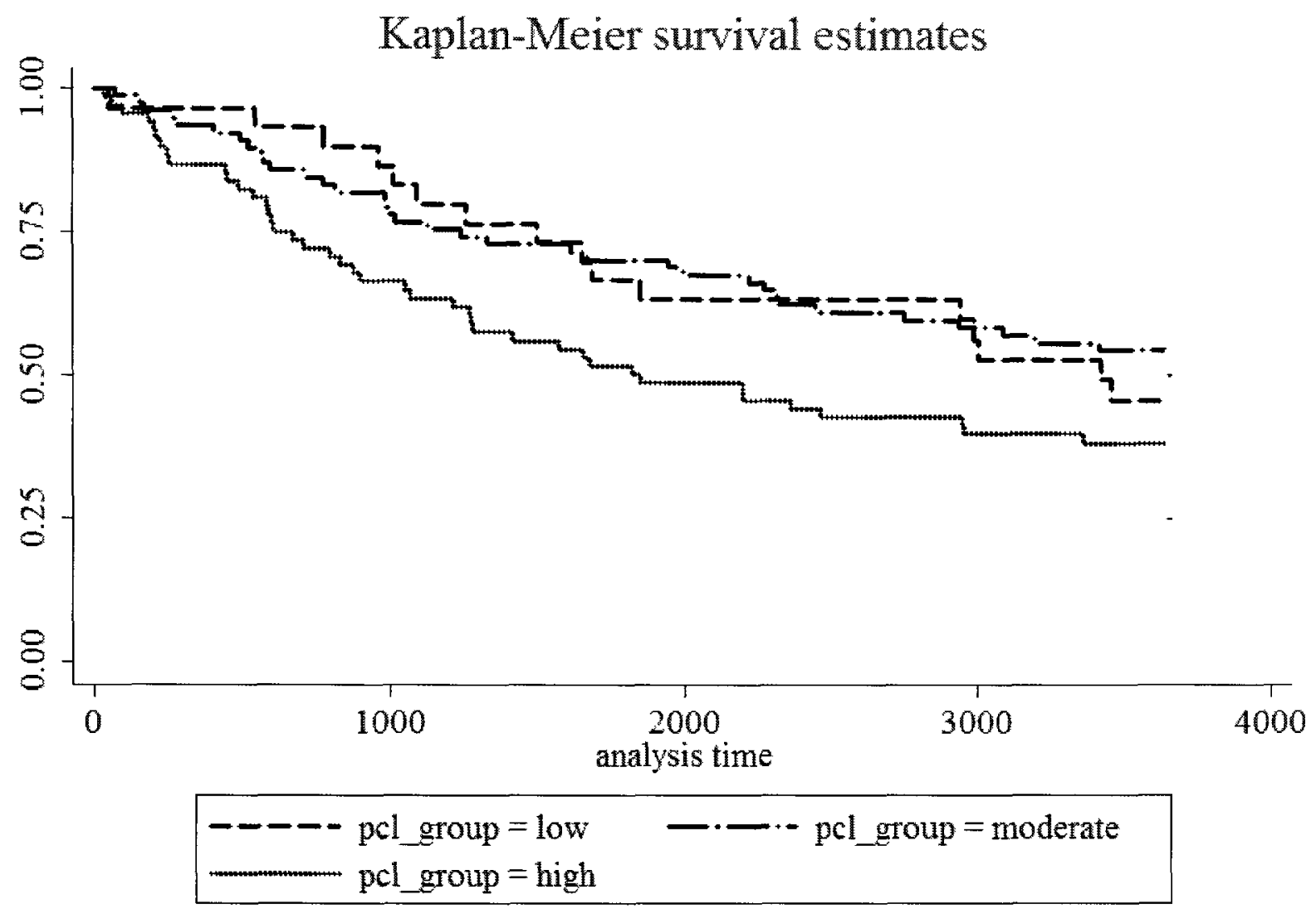


Figure 12.

Kaplan-Meier Graph - One Year Violent Recidivism for SAVRY Groups

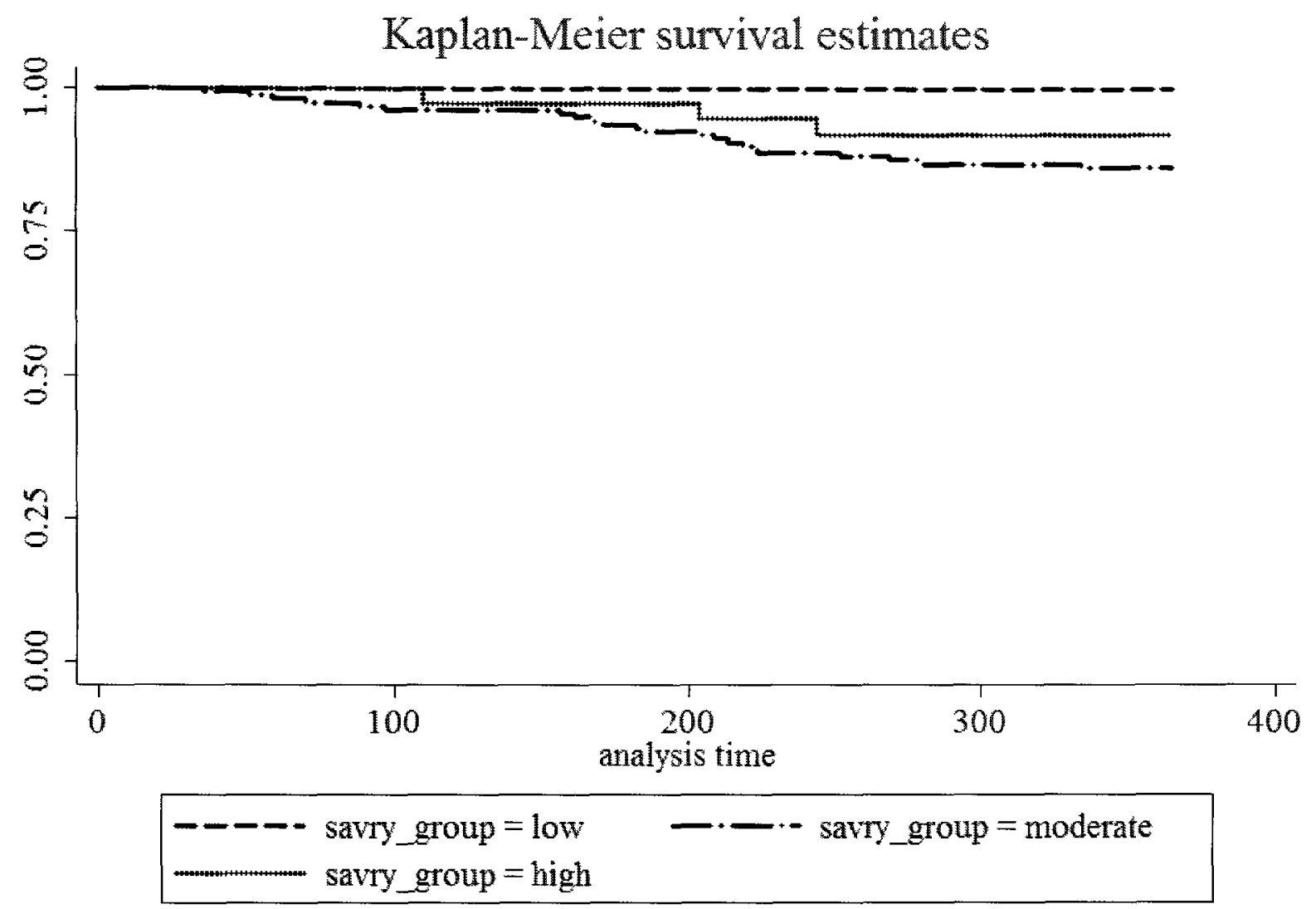


Figure 13.

Kaplan-Meier Graph - Five Year Violent Recidivism for SAVRY Groups

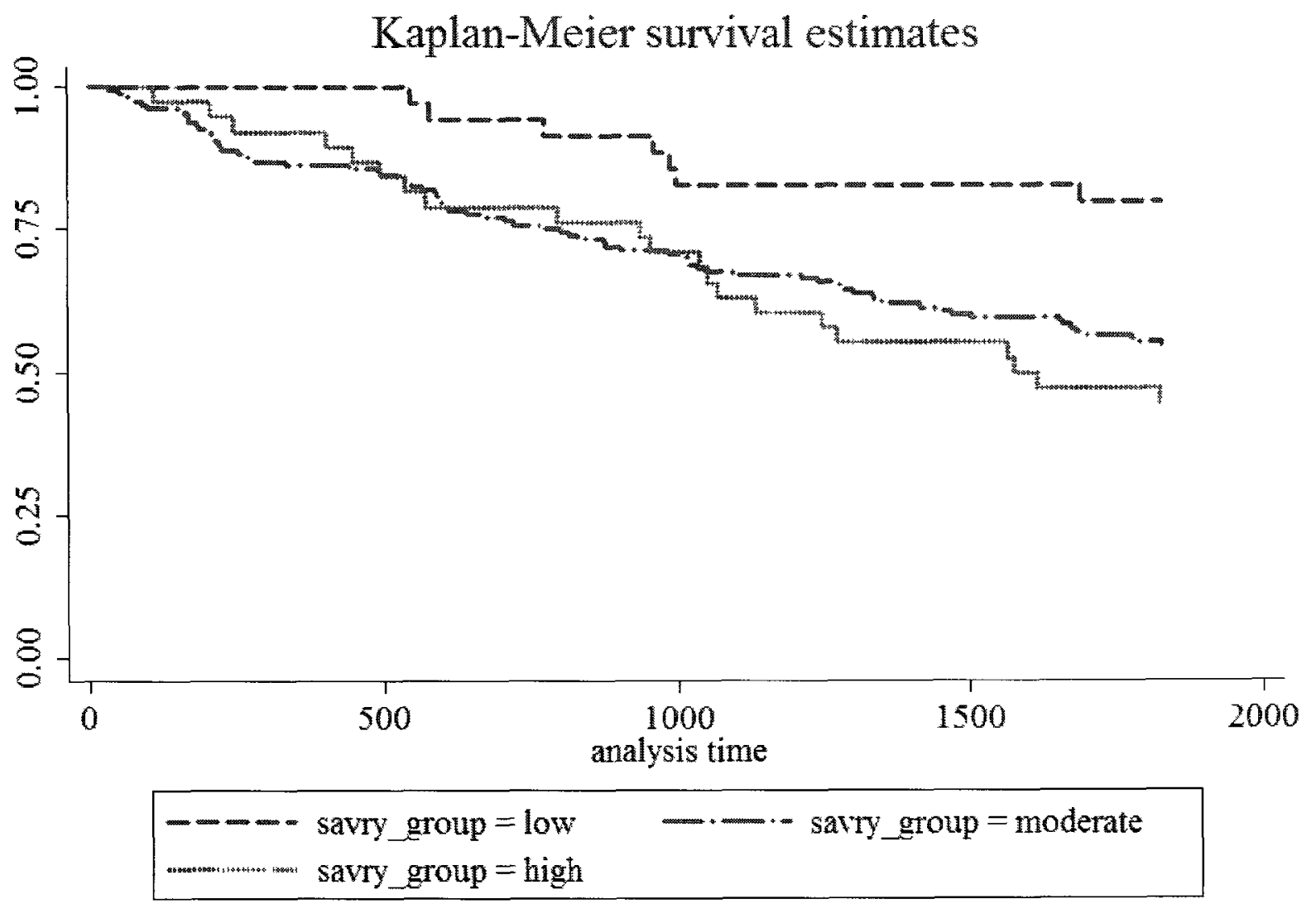


Figure 14.

Kaplan-Meier Graph - Ten Year Violent Recidivism for SAVRY Groups

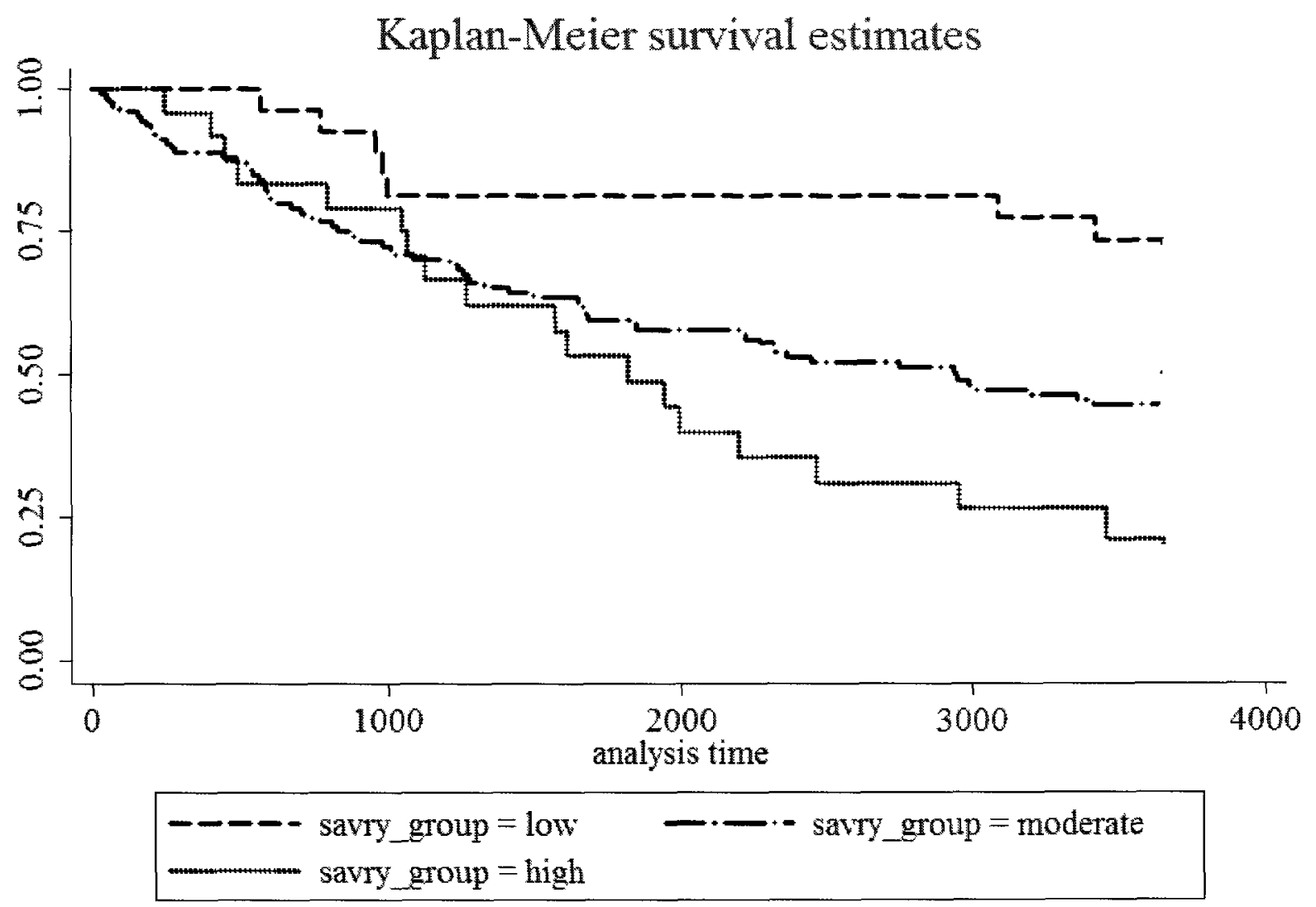




\section{Role of Protective Factors in Criminal Desistence - Objective Three}

To examine whether protective factors as assessed by the SAVRY would have an impact on re-offending for participants who were convicted of a criminal offence during the follow-up period, several analytic techniques were utilized.

As a first step, the frequency of the SAVRY protective items were examined for the entire sample and then for each offence type. This examination revealed that a large proportion of participants were found to have either no or only one protective factor $(63.3 \%)$ and only $0.4 \%$ (1 participant) was found to have all 6 protective factors. For youth who were found to have 4 or more protective factors $(7.2 \%$ or 17 participants), $58.8 \%$ had recidivated non-violently during the follow-up period and $35.3 \%$ had recidivated violently. Of youth who had none or only one protective factor, $80.4 \%$ had re-offended non-violently and $70.3 \%$ had re-offended violently (see Table 27).

Table 27

Base Rates of Re-Offending for Non-Violent and Violent Offences Based on the Number of Protective Factors Present

\begin{tabular}{lcccc}
\hline \# of Protective Factors Present & $n$ & General & Non-violent & Violent \\
& & & & \\
None & 80 & 87.5 & 82.5 & 76.3 \\
One & 68 & 83.8 & 77.9 & 63.2 \\
Two & 45 & 71.1 & 71.1 & 60.0 \\
Three & 24 & 75.0 & 70.8 & 37.5 \\
Four & 11 & 54.5 & 54.5 & 45.5 \\
Five & 5 & 60.0 & 60.0 & 0 \\
Six & 1 & 100.0 & 100.0 & 100.0 \\
& & & & \\
\hline
\end{tabular}


Table 28 illustrates that persisters and desisters of non-violent offending do not significantly differ on whether each of the protective factors, as assessed by the SAVRY were either absent or present. Having a positive attitude toward intervention and authority was significant at the $p<.01$ level; however after applying the Bonferroni correction, this association became non-significant. When persisters and desisters of violent offending were compared on each of the SAVRY protective items, two comparisons were significant. Desisters of violent offending were more likely to have a positive attitude toward intervention and authority $(22.1 \%$ versus $9.2 \%$ of persisters). Further, desisters were also more likely to have a strong commitment to school $(28.7 \%$ versus $9.7 \%$ of persisters $)$. These comparisons were significant even after the Bonferroni correction was applied. 
Persistence and Desistence of Offending 104

Table 28

Youth who Either Persisted or Desisted From Non-Violent Offending: A Comparison of the SAVRY Protective Items

\begin{tabular}{lccccc}
\hline Protective Factor & & $\begin{array}{c}\text { Persisters } \\
\% \\
n=178\end{array}$ & $\begin{array}{c}\text { Desisters } \\
\% \\
n=57\end{array}$ & $\chi^{2}$ & $p$ \\
& & & & & \\
& Absent & 63.3 & 47.8 & 3.44 & .06 \\
Pro-social involvement & Present & 36.7 & 52.2 & & \\
& Absent & 76.7 & 66.7 & 2.18 & .14 \\
Strong social support & Present & 23.3 & 33.3 & & \\
& Absent & 62.1 & 57.4 & 0.38 & .54 \\
Strong attachments and bonds & Present & 37.9 & 42.6 & & \\
& Absent & 89.5 & 75.0 & 6.64 & .01 \\
Positive attitude toward & Present & 10.5 & 25.0 & & \\
intervention \& authority & Absent & 81.8 & 76.4 & 0.80 & .37 \\
\multirow{2}{*}{ Strong commitment to school } & Present & 18.2 & 23.6 & & \\
& Absent & 95.7 & 93.8 & 0.20 & .66 \\
Resilient personality traits & Present & 4.3 & 6.3 & & \\
\hline
\end{tabular}

Note: after Bonferroni correction of $.05 / 6=p<.008$, none of the above comparisons were significant. 
Table 29

Youth who Either Persisted or Desisted From Violent Offending: A Comparison of the SAVRY

Protective Items

\begin{tabular}{|c|c|c|c|c|c|}
\hline Protective Factor & & $\begin{array}{c}\text { Persisters } \\
\% \\
n=146 \\
\end{array}$ & $\begin{array}{c}\text { Desisters } \\
\% \\
n=89 \\
\end{array}$ & $\chi^{2}$ & $p$ \\
\hline Pro-social involvement & $\begin{array}{l}\text { Absent } \\
\text { Present }\end{array}$ & $\begin{array}{c}66.14 \\
33.9\end{array}$ & $\begin{array}{l}49.3 \\
50.7\end{array}$ & 5.15 & .02 \\
\hline Strong social support & $\begin{array}{l}\text { Absent } \\
\text { Present }\end{array}$ & $\begin{array}{l}79.3 \\
20.7\end{array}$ & $\begin{array}{l}65.9 \\
34.1\end{array}$ & 5.07 & .02 \\
\hline Strong attachments and bonds & $\begin{array}{l}\text { Absent } \\
\text { Present }\end{array}$ & $\begin{array}{l}65.5 \\
34.5\end{array}$ & $\begin{array}{l}53.0 \\
47.0\end{array}$ & 3.47 & .06 \\
\hline $\begin{array}{l}\text { Positive attitude toward } \\
\text { intervention \& authority }\end{array}$ & $\begin{array}{l}\text { Absent } \\
\text { Present }\end{array}$ & $\begin{array}{c}90.8 \\
9.2\end{array}$ & $\begin{array}{l}77.9 \\
22.1\end{array}$ & 7.05 & .008 \\
\hline Strong commitment to school & $\begin{array}{l}\text { Absent } \\
\text { Present }\end{array}$ & $\begin{array}{c}90.3 \\
9.7\end{array}$ & $\begin{array}{l}71.3 \\
28.7\end{array}$ & 13.97 & .000 \\
\hline Resilient personality traits & $\begin{array}{l}\text { Absent } \\
\text { Present }\end{array}$ & $\begin{array}{c}96.8 \\
3.2\end{array}$ & $\begin{array}{c}92.5 \\
7.5\end{array}$ & 1.42 & .23 \\
\hline
\end{tabular}

Note: bold denotes significance after Bonferroni correction of $p<.05 / 6=.008$.

To further distinguish whether youth who had a particular protective factor would differ from those who did not have the protective factor present on the number of non-violent and violent reconvictions, several t-tests were computed. The numbers of non-violent and violent reconvictions, subsequent to their index release were compared for each protective factor. As illustrated in Table 30, youth who had strong social support also had fewer number of nonviolent reconvictions $(M=7.4, S D=9.7)$ than those who did not have strong social support $(M=$ 14.7, $S D=17.1$ ). Further, having a positive attitude also meant that fewer non-violent 
convictions were recorded $(M=4.7, S D=6.8)$ compared with those who did not have a positive attitude $(M=14.6, S D=16.6)$. Youth who had a strong commitment to school were found to have committed fewer violent offences $(M=0.7, S D=1.2)$ than those who were not seen to have a high commitment to school $(M=2.9, S D=4.7)$. 
Table 30

T-tests Comparing Number of Non-Violent and Violent Offences for Each SAVRY Protective Item

\begin{tabular}{|c|c|c|c|c|c|}
\hline Protective Item & Absent & Present & $t$ & $\mathrm{df}$ & $p$ \\
\hline & \multicolumn{5}{|c|}{ Non-Violent Offending } \\
\hline Pro-social involvement & $12.4(14.7)$ & $9.2(12.0)$ & 1.55 & 183 & .12 \\
\hline Strong social support & $14.7(17.1)$ & $7.4(9.7)$ & 3.14 & 228 & .002 \\
\hline Strong attachments and bonds & $14.7(16.7)$ & $10.1(14.0)$ & 2.19 & 226 & .03 \\
\hline $\begin{array}{l}\text { Positive attitude toward } \\
\text { intervention } \mathcal{\&} \text { authority }\end{array}$ & $14.6(16.6)$ & $4.7(6.8)$ & 3.22 & 217 & .001 \\
\hline Strong commitment to school & $13.8(15.9)$ & $8.9(15.1)$ & 1.88 & 229 & .06 \\
\hline \multirow[t]{2}{*}{ Resilient personality traits } & $12.8(15.7)$ & $4.6(5.6)$ & 1.39 & 145 & .17 \\
\hline & \multicolumn{5}{|c|}{ Violent Offending } \\
\hline Pro-social involvement & $2.2(3.4)$ & $2.1(5.6)$ & 0.15 & 183 & .88 \\
\hline Strong social support & $2.8(4.9)$ & $1.4(2.1)$ & 2.11 & 228 & .04 \\
\hline Strong attachments and bonds & $2.9(4.9)$ & $1.9(3.5)$ & 1.58 & 226 & .12 \\
\hline $\begin{array}{l}\text { Positive attitude toward } \\
\text { intervention } \& \text { authority }\end{array}$ & $2.9(4.7)$ & $0.8(1.3)$ & 2.32 & 217 & .02 \\
\hline Strong commitment to school & $2.9(4.7)$ & $0.7(1.2)$ & 3.10 & 229 & .002 \\
\hline Resilient personality traits & $2.4(4.8)$ & $1.6(3.3)$ & 0.46 & 145 & .65 \\
\hline
\end{tabular}

In an additional analysis, the participants' score on the PCL:YV and SAVRY were also compared to discern if youth who were categorized into low, moderate and high scoring groups would differ on the number of protective items present. To achieve this, two separate oneway 
analysis of variance (ANOVA) tests were calculated. The first test indicated that there was a significant main effect, $F(2,231)=17.08, p<.001$, indicating that the PCL:YV groups significantly differed on the number of protective items present. Post hoc pairwise comparisons indicated that those in the high scoring PCL:YV group had lower protective scores $(M=0.8, S D$ $=0.9)$ than those in the low scoring PCL:YV group $(M=2.1, S D=1.6)$. The medium $(M=1.3$, $S D=1.2$ ) scoring PCL:YV group also significantly differed from the low scoring PCL:YV group. All comparisons were significant at the $p<.001$ level. When the SAVRY groups were examined to discern if differences across groups would emerge on the number of protective items present, the ANOVA revealed that a significant main effect was found, $F(2,231)=47.08$, $p<.001$. Post hoc pairwise comparisons illustrated that those in the high scoring SAVRY risk group $(M=0.3, S D=0.5)$ differed from those in the moderate scoring SAVRY risk group $(M=$ $1.2, S D=1.2)$ and those in the low scoring SAVRY risk group $(M=2.8, S D=1.4)$. Those in the moderate scoring SAVRY risk group also significantly differed from those in the low scoring SAVRY risk group. All comparisons were significant at the $p<.001$ level.

Individual protective factors were evaluated to discern whether they were predictive of non-violent or violent desistence. Recall from objective two that the overall protective score was related to general recidivism at the 10 -year follow-up period, (AUC $=.67, p<.001)$ and that for all other outcome variables, the AUC was considered to be low or non-significant. Therefore, the individual protective items were examined utilizing a univariate and multivariate logistic regression to explore the unique and simultaneous contribution these items have with non-violent and violent offending. The protective factor was reversed coded for all logistic regression analyses to reflect that higher scores meant the youth had few protective items present. This coding allowed comparisons to risk measures in which higher scores reflect greater level of risk. 
Table 31 provides the results of the univariate logistic regressions for any non-violent recidivism. These analyses illustrate that for non-violent offending, youth who served as having a positive attitude towards intervention and authority were significantly less likely to have committed a non-violent offence during the entire follow-up period, $\mathrm{B}=-1.04, \mathrm{SE}=0.42$, Wald $=6.26, \mathrm{p}<.01, \operatorname{Exp}[\mathrm{B}]=0.35$. No other protective items were significant predictors of nonviolent offending.

\section{Table 31}

Univariate Logistic Regression for SAVRY Protective Items Predicting Any Non-Violent Recidivism

Protective Item

$B \quad$ SE $\quad$ Wald $p \quad \operatorname{Exp}[B]$

\begin{tabular}{cc}
$\operatorname{Exp}[B]$ & $95 \% C I$ \\
\hline Lower upper
\end{tabular}

Pro-social involvement $.63 \quad .345$

3.39

.06

1.88

.96

3.69

Strong social support

$-.49$

.34

$2.16 \quad .14 \quad .61$

.31

1.18

Strong attachments and bonds

$-.19 \quad .32$

$.38 \quad .54$

.82

.44

1.53

Positive attitude toward intervention \& authority

$\begin{array}{lllllllll}-1.04 & .42 & 6.26 & .01 & .35 & .16 & & .80\end{array}$

Strong commitment to school $-.33$ $-33$

.37

.79

.37

.72

Resilient personality traits

$$
-.38
$$

.86

The omnibus model containing all six protective factors was then examined. The results indicate that the model was almost statistically related to any non-violent offences during the entire follow-up period, $\chi^{2}(7)=13.51, p=.06$, Nagelkerke $R^{2}=.18$, Hosmer and Lemeshow 
indicated a good fit, $\chi^{2}(8)=6.74, p=.57$, with $77.5 \%$ of the cases correctly identified, however none of the predictor variables were statistically significant (see Table 32).

Table 32

Multivariate Logistic Regression for SAVRY Protective Items Predicting Any Non-Violent Recidivism

\begin{tabular}{|c|c|c|c|c|c|c|c|}
\hline \multirow[t]{2}{*}{ Protective Item } & \multirow[t]{2}{*}{$B$} & \multirow[t]{2}{*}{$S E$} & \multirow[t]{2}{*}{ Wald } & \multirow[t]{2}{*}{$p$} & \multirow[t]{2}{*}{$\operatorname{Exp}[B]$} & \multicolumn{2}{|c|}{$\operatorname{Exp}[B] 95 \% C I$} \\
\hline & & & & & & Lower & upper \\
\hline Pro-social involvement & .74 & .55 & 1.81 & .18 & 2.10 & .71 & 6.19 \\
\hline Strong social support & .77 & .72 & 1.14 & .29 & 2.15 & .53 & 8.82 \\
\hline Strong attachments and bonds & -1.09 & .69 & 2.49 & .12 & .34 & .09 & 1.30 \\
\hline $\begin{array}{l}\text { Positive attitude toward } \\
\text { intervention } \& \text { authority }\end{array}$ & .42 & .66 & .41 & .52 & 1.52 & .42 & 5.48 \\
\hline Strong commitment to school & -.89 & .65 & 1.9 & .17 & .41 & .12 & 1.46 \\
\hline Resilient personality traits & -1.21 & 1.18 & 1.05 & .31 & .30 & .03 & 3.03 \\
\hline
\end{tabular}

Next, the individual SAVRY protective items were examined for any violent reconviction during the entire follow-up period. The results indicate that several of the protective items were indicative of violent offending. Having a strong social support, $\mathrm{B}=-.69, S E=0.31$, Wald $=4.98$, $p<.03$, having a positive attitude toward intervention and authority, $\mathrm{B}=-1.03, S E=0.4$, Wald $=$ $6.67, p<.01$, and having a strong commitment to school, $\mathrm{B}=-1.41, S E=0.35, \mathrm{Wald}=16.3, p<$ .001 , were all related to the prediction of violent desistence. Interestingly, having a pro-social involvement was a significant predictor of violent persistence, $\mathrm{B}=0.69, S E=0.31$, Wald $=$ 
$5.09, p<.02$, with an $\operatorname{Exp}[\mathrm{B}]$ of 2.0 , indicating that those with this item present were $100 \mathrm{x}$ more likely to commit a violent offence (see Table 33 ).

Table 33

Univariate Logistic Regression for SAVRY Protective Items Predicting Any Violent Recidivism

Protective Item

$$
B \quad S E \quad \text { Wald } p \quad \operatorname{Exp}[B] \frac{\operatorname{Exp}[B] 95 \% C I}{\text { Lower upper }}
$$

\begin{tabular}{|c|c|c|c|c|c|c|c|}
\hline Pro-social involvement & .69 & .31 & 5.09 & .02 & 2.00 & 1.09 & 3.66 \\
\hline Strong social support & -.69 & .31 & 1.98 & .03 & .50 & .27 & .92 \\
\hline Strong attachments and bonds & -.52 & .28 & 3.44 & .06 & .59 & .34 & 1.03 \\
\hline $\begin{array}{l}\text { Positive attitude toward } \\
\text { intervention } \& \text { authority }\end{array}$ & -1.03 & .40 & 6.67 & .01 & .36 & .16 & .78 \\
\hline Strong commitment to school & -1.14 & .35 & 16.29 & .001 & .24 & .12 & .48 \\
\hline Resilient personality traits & -.91 & .78 & 1.34 & .24 & .40 & .09 & 1.87 \\
\hline
\end{tabular}

The omnibus model examining all protective factors together to discern whether persisters would differ from desisters indicated that, overall, the model was significant, $\chi^{2}(7)=$ $15.55, p<.03$, with the factors accounting for $18 \%$ of the variance explained (Nagelkerke $R^{2}$ ) and the Hosmer and Lemeshow test indicated the model was a good fit, $\chi^{2}(8)=8.84, p=.36$. The model correctly classified $72.1 \%$ of youth. Table 30 shows that the only significant predictor of violence persistence to emerge was having a strong attachment to school, with those who have 
the item present being 3.48 times more likely to violently persist in their offending (see Table 34).

Table 34

Multivariate Logistic Regression for SAVRY Protective Items Predicting Any Violent Recidivism

Protective Item $\quad B \quad S E$ Wald $\quad p \quad \operatorname{Exp}[B] \frac{\operatorname{Exp[B]95\% CI}}{\text { Lower upper }}$

$\begin{array}{lccccccc}\text { Pro-social involvement } & .24 & .50 & .23 & .63 & 1.27 & .47 & 3.41 \\ \text { Strong social support } & .68 & .61 & 1.23 & .27 & 1.98 & .60 & 6.58 \\ \text { Strong attachments and bonds } & -.78 & .59 & 1.75 & .19 & .46 & .14 & 1.46 \\ \begin{array}{l}\text { Positive attitude toward } \\ \text { intervention \& authority }\end{array} & .19 & .60 & .10 & .75 & 1.21 & .37 & 3.93 \\ \text { Strong commitment to school } & 1.24 & .53 & 5.40 & .02 & 3.48 & 1.21 & 9.97 \\ \text { Resilient personality traits } & -.52 & .95 & .30 & .58 & .59 & .09 & 3.81 \\ \end{array}$

Next, logistic regression analyses were computed to evaluate the incremental validity of the protective factor score with each of the PCL:YV and SAVRY risk ratings. When PCL:YV total scores were entered at step 1, when predicting any non-violent offence (binary coded 0/1), the overall model was not significant, $\chi^{2}(1)=0.14, p=.70, R^{2}$ (Nagelkerke) $=.001, \mathrm{~B}=.01$, Wald $=.15$, indicating that scores on the PCL:YV were not related to non-violent offending. When the protective factor was entered at step 2, the model became significant, the model became significant, $\chi^{2}(1)=4.94, p<.03, R^{2}=.032$, with $75.6 \%$ of youth correctly classified. The $R^{2}$ change indicated that $3 \%$ of the variance was accounted for by the protective score and the negative $B$ of $-.28, \operatorname{Exp}[\mathrm{B}]=.76$ indicates that the higher the protective score, the less likely 
the youth would recidivate non-violently. The total PCL:YV score variable was not a significant predictor in this model. When the model was reversed and SAVRY protective was added at step 1, followed by the PCL:YV total score at step 2, the $R^{2}$ change indicated that by adding the PCL:YV total score only accounted for $0.2 \%$ of the variance explained. The model was significant at step $1, \chi^{2}(1)=4.72, p<.03$, with the protective score illustrating that having higher protective scores was related to desistence, $\mathrm{B}=-.25, \mathrm{Exp}[\mathrm{B}]=.78$ (see Table 35 ).

In examining the incremental predictive validity for violent recidivism of the PCL:YV over the SAVRY protective rating, when the PCL:YV total score was entered at step 1, the model was significant, $\chi^{2}(1)=10.25, p<.0014, R^{2}=.058$ and the Homer and Lemeshow goodness of fit indicating the model was a good fit, $\chi^{2}(8)=6.05, p=.64$, with $60.3 \%$ of youth correctly classified. When SAVRY protective score was entered at step 2 , the model remained significant, $\chi^{2}(8)=9.73, p<.002$ and the $R^{2}$ was .113 indicating that by adding the protective score, $5.5 \%$ of added variance was explained. The model now correctly classified $66.2 \%$ of youth. When the model was reversed and the SAVRY protective score was entered at step 1, the model was significant, $\chi^{2}(1)=17.14, p<.001$ with $9.6 \%$ of the variance explained. The Homer and Lemeshow goodness of fit test indicated the model was a good fit, $\chi^{2}(3)=1.32, p=.72$ with $67.1 \%$ of youth correctly classified. When the PCL:YV total score was entered at step 2 , the model was no longer significant, $\chi^{2}(1) 2.84, p<.09$ and the addition of the PCL:YV total score only accounted for $1.6 \%$ of additional variance explained (see Table 35 ). 
Table 35

Incremental Validity of the PCL:YV over the SAVRY Protective Rating for the Prediction of Non-Violent and Violent Offending

\begin{tabular}{|c|c|c|c|c|c|c|}
\hline \multirow[t]{2}{*}{ Variable } & \multirow[t]{2}{*}{ Wald } & \multirow[t]{2}{*}{ df } & \multirow{2}{*}{$p$} & \multirow[t]{2}{*}{$\operatorname{Exp}[B]$} & \multicolumn{2}{|c|}{$\operatorname{Exp}[B] 95 \% C I$} \\
\hline & & & & & Lower & upper \\
\hline & \multicolumn{6}{|c|}{ Non-Violent Offending } \\
\hline Step 1: PCL:YV total & .15 & 1 & .70 & 1.00 & .97 & 1.05 \\
\hline Step 2: PCL:YV total & .36 & 1 & .55 & .99 & .95 & 1.03 \\
\hline SAVRY protective & 4.89 & 1 & .03 & .76 & .59 & .97 \\
\hline Step 1: SAVRY protective & 4.78 & 1 & .03 & .78 & .63 & .98 \\
\hline Step 2: SAVRY protective & 4.89 & 1 & .03 & .76 & .59 & .97 \\
\hline \multirow[t]{2}{*}{ PCL:YV total } & .36 & 1 & .55 & .99 & .95 & 1.03 \\
\hline & \multicolumn{6}{|c|}{ Violent Offending } \\
\hline Step 1: PCL:YV total & 9.88 & 1 & .002 & 1.06 & 1.02 & 1.09 \\
\hline Step 2: PCL:YV total & 2.84 & 1 & .09 & 1.03 & 1.00 & 1.07 \\
\hline SAVRY protective & 9.24 & 1 & .002 & .70 & .55 & .88 \\
\hline Step 1: SAVRY protective & 15.74 & 1 & .000 & .65 & .52 & .80 \\
\hline Step 2: SAVRY protective & 9.24 & 1 & .002 & .70 & .55 & .88 \\
\hline PCL:YV total & 2.84 & 1 & .09 & 1.03 & 1.00 & 1.07 \\
\hline
\end{tabular}

When SAVRY risk rating was entered at step 1 for non-violent offending, it was a significant predictor of persistence, $R^{2}=.03, \mathrm{~B}=.05, \mathrm{Wald}=5.02, p<.03$, indicating that having a high risk score was related to non-violent persistence. When the protective factor was entered at step 2, SAVRY risk did not remain significant and the protective factor was not a significant predictor, $\mathrm{B}=-.15, \mathrm{Wald}=1.16, p=.28, R^{2}=.04$ with $0.7 \%$ of added variance explained. When the model was reversed and SAVRY protective was entered at step 1, it was 
found to be a significant predictor of non-violent desistence, $\mathrm{B}=-.25$, Wald $=4.78, p<.03, R^{2}=$ .03 . When SAVRY risk was entered at step 2 , both the protective and risk ratings were nonsignificant, however the addition of the SAVRY risk score accounted for $1 \%$ in explained variance (see Table 36).

The last model that was examined was the incremental validity of the SAVRY risk over the SAVRY protective score in the prediction of violent offending. The first model explored SAVRY risk entered at step 1 . This revealed a significant model, $\chi^{2}(1)=19.20, p<.001$ with $10.7 \%$ of the variance explained and the Homer and Lemeshow test indicating a good fitting model, $\chi^{2}(8)=7.47, p=.49$ with $64.5 \%$ of youth correctly classified. When the protective score was entered at step 2 , the model remained significant, $\chi^{2}(1)=4.19, p<.04$ with $2.3 \%$ of additional variance explained and $67.1 \%$ of youth correctly classified. When the models were reversed and the protective item was entered at step 1, the model was significant, $\chi^{2}(1)=17.14$, $p<.001$ with an $R^{2}$ of .096 and a goodness of fit test of $\chi^{2}(3)=1.32, p=.72$ as measured by the Homer and Lemeshow test. The model correctly classified $67.1 \%$ of youth. When SAVRY risk was entered at step 2 , the model remained significant, $\chi^{2}$ (1) $6.24, p<.02$ with $3.4 \%$ of additional variance explained and the same amount of youth correctly classified (67.1\%; see Table 36$)$. 
Persistence and Desistence of Offending 116

Table 36

Incremental Validity of the SAVRY Risk Rating over the SAVRY Protective Rating for the Prediction of Non-Violent and Violent Offending

\begin{tabular}{|c|c|c|c|c|c|c|}
\hline \multirow[t]{2}{*}{ Variable } & \multirow[t]{2}{*}{ Wald } & \multirow[t]{2}{*}{$\mathrm{df}$} & \multirow[t]{2}{*}{$p$} & \multirow[t]{2}{*}{$\operatorname{Exp}[B]$} & \multicolumn{2}{|c|}{$\operatorname{Exp}[B] 95 \% C I$} \\
\hline & & & & & Lower & upper \\
\hline & \multicolumn{6}{|c|}{ Non-Violent Offending } \\
\hline Step 1: SAVRY risk total & 5.01 & 1 & .03 & 1.05 & 1.01 & 1.09 \\
\hline Step 2: SAVRY risk total & 1.58 & 1 & .21 & 1.03 & .98 & 1.08 \\
\hline SAVRY protective & 1.16 & 1 & .28 & .86 & .66 & 1.13 \\
\hline Step 1: SAVRY protective & 4.78 & 1 & .03 & .78 & .63 & .98 \\
\hline Step 2: SAVRY protective & 1.16 & 1 & .28 & .86 & .66 & 1.13 \\
\hline \multirow[t]{2}{*}{ SAVRY risk total } & 1.58 & 1 & .21 & 1.03 & .98 & 1.08 \\
\hline & \multicolumn{6}{|c|}{ Violent Offending } \\
\hline Step 1: SAVRY risk total & 17.31 & 1 & .000 & 1.09 & 1.04 & 1.13 \\
\hline Step 2: SAVRY risk total & 6.03 & 1 & .02 & 1.06 & 1.01 & 1.11 \\
\hline SAVRY protective & 4.08 & 1 & .04 & .77 & .60 & .99 \\
\hline Step 1: SAVRY protective & 15.74 & 1 & .000 & .65 & .52 & .80 \\
\hline Step 2: SAVRY protective & 4.08 & 1 & .04 & .77 & .60 & .99 \\
\hline SAVRY risk total & 6.03 & 1 & .02 & 1.06 & 1.01 & 1.11 \\
\hline
\end{tabular}




\section{Chapter IV: Discussion}

The objective of this dissertation was three-fold: to distinguish if social, personality, clinical or background factors would differ between youth who persisted in their offending post release for youth offences versus those who desisted in offending; to determine the predictiveness of two assessment inventories and the number of conduct disorder symptoms on future offending, both in terms of probability and imminence; and finally, to discern if certain factors, particularly protective factors, alone or in combination with risk factors were related to either persistence or desistence of offending. Utilizing a prospective research design, the current study examined 235 male youth offenders who were either serving or were remanded to custody in a youth detention facility. The youth were followed once released from custody to determine the number, type, and dates of their offences. Risk factors were examined through chi-square analyses; prediction of future offending was explored through Cox regressions, Kaplan-Meier curves, and ROC analyses. Protective factors were examined through ROC analyses, Cox regressions and logistic regressions. Overall, the results of the current study indicate that youth desisters and persisters of criminal offending are distinguishable on several key indicators that may help with risk management strategies when dealing with youth who are in conflict with the law.

The sample's overall score on both the PCL:YV and SAVRY were consistent with what has been reported elsewhere in the literature with a forensic sample of youth offenders (Corrado et al., 2004; Edens et al., 2007; Gretton et al., 2004; Rennie \& Dolan, 2008; Salekin, 2008; Viljoen et al., 2008). The internal consistency found for both the PCL:YV and SAVRY were comparable to other studies (Forth et al., 2003; Vincent, et al., 2011; Vitacco, Neumann, \& Caldwell, 2010; Welsh et al., 2008). Offence base rates that were observed in the present study 
were also consistent with other studies examining youth offenders serving custodial sentences or on remand (Vincent et al., 2011; Vincent, Odgers, McCormick, \& Corrado, 2008). The base rates in the current study were less than what was found in Gretton et al. (2004), however, given the three samples in the present study had differing follow-up times, this is not surprising.

Several factors have been identified in the literature to be associated with psychopathy, including criminal behaviour (Leistico et al., 2008), violent offending (Porter et al., 2004), criminal recidivism (Edens et al., 2007; Leistico et al., 2008), conduct disorder (Forth \& Burke, 1998), antisocial behaviour (Vincent et al., 2003), having negative family factors (Campbell et al., 2004; Marshall \& Cooke, 1999), having poor academic achievement (Campbell et al., 2004), and having antisocial peers and associates (Barry et al., 2008). Many studies which have examined related risk factors with childhood psychopathy have used retrospective case-control designs, with few longitudinal studies completed to date. Nonetheless, it is imperative that we investigate factors which may cause or influence the development of psychopathy. Campbell et al. (2004) retrospectively examined youth who scored high on psychopathic traits and found these individuals to be more likely to have suffered physical abuse and separation from their parents when compared with non-psychopaths. Marshall and Cooke (1999) also found that high scoring psychopathic offenders were more likely to have experienced parental indifference or neglect, poor parental supervision and poor parental discipline. Forth and Burke (1998) reported that amongst their sample of 106 male youth offenders and 50 community youth, having psychopathic traits was associated with negative family factors such as parental deviance, parental drug abuse, separation from parent and inconsistent discipline.

The research literature has identified other key factors, such as social, clinical, familial and background indicators that are known correlates of antisocial behaviour, but few have 
examined if those who persist in their offending differ from those who desist on these key factors. The current study sought to determine if differing patterns would emerge amongst these two groups. The results were quite different based upon the type of re-offence committed. Youth who went on to commit non-violent offences did not differ from youth who desisted in nonviolent offending on these key indicators. However, youth who went on to re-offend violently differed on several key factors from those who desisted in violent offending. Persisters were found to have higher psychopathy scores and have higher antisocial scores (factor 4 ) on the PCL:YV. This group also was found to have higher SAVRY risk scores, higher SAVRY historical and SAVRY clinical scores and were found to have lower SAVRY protective scores. Youth who violently persisted were also more likely to have higher number of conduct disorder symptoms, more likely to have substance abuse issues and have less commitment to school.

Previous research has identified that youth offenders who persist in criminal offending often lack positive social support or have adequate social ties, resulting in poor attachment and social integration. Laub and Sampson (2003) found that adults who had strong ties to workplace and family were less likely to re-offend. In a longitudinal study, Wileman, Gullone, and Moss (2007) followed 100 male juvenile offenders from 1980 until 2002. The results of their study indicated that when youth were identified at a young age to be in need of crime prevention initiatives and were identified as being at risk of future offending, reductions in criminal offending were observed. Wileman et al.'s (2007) study and the results of the current study are suggestive of the need to identify, as early as possible, negative dynamic risk indicators in a youth's life that may play a role in fostering and continuing a maladaptive lifestyle that is characterized by criminal offending. Youth who were persistent in violent offending show clear 
Persistence and Desistence of Offending 120

differences on certain key social and clinical factors that if left untreated, may predispose and increase the likelihood of a trajectory of a criminal career.

The main goal of objective two was to examine the PCL:YV and SAVRY separately and in conjunction with each other to determine the ability to predict future non-violent and violent offending for youth and adult offences. As a first step, psychopathy scores and risk of violence scores were examined to see their respective relation with other factors. The current study found that psychopathy scores were significantly related to the number of conduct disorder symptoms $(r=.41)$. All youth in the present study who were classified as being in the high psychopathic group $(\geq 30$ ) were found to also meet the diagnostic criteria for conduct disorder, whereas $36.4 \%$ of those meeting the diagnosis of conduct disorder were also found to score high on psychopathy. This finding is similar to what Sevecke et al. (2009) reported. This provides further support that although the two disorders are similar and do overlap, they constitute two distinct syndromes.

Total PCL:YV scores were also found to be significantly related to substance abuse ( $r=$ .23). Ninety-three percent of the participants in the high psychopathic group reported having issues relating to substance use, while only $68.9 \%$ of those in the low group reported such problems. This is consistent with past research indicating that those with psychopathic traits were more likely to also exhibit substance abuse problems (Loney et al., 2007; Neumann \& Hare, 2008). The results also showed that psychopathic traits were associated with having ever experience violence $(r=.23)$. Slightly over half of participants $(51.2 \%)$ of youth who fell in the high psychopathy group reported that they experienced violence at some point in their lives. Those in the low group were found to have experienced violence in $21 \%$ of the cases. Another risk factor found to be associated with psychopathy scores was having a low commitment to 
school $(r=.43)$. This finding is consistent with previous studies (Brandt et al., 1992; Campbell et al., 2004). Almost all the youth in the present study who scored high on psychopathic traits (98.7\%) were found to have a low commitment to school. This risk factor has been found to be associated with early-onset offenders (Stattin \& Magnusson, 1995).

When age of first known offence was examined, a significant relationship was noted with psychopathy scores $(r=-.40)$. Given these ages were based on self-report and not official crime reports, leads us to conclude that criminal offending begins much earlier for those with psychopathic traits than those scoring low. Criminal offending often begins long before official criminal sanctions are given. Those with high psychopathic traits were found to start offending two full years, on average, before those scoring low and a year earlier than those scoring in the moderate range. The link between criminal behaviour and psychopathy is well established (Farrington, 1992; Forth et al., 2003; Hare et al., 2000; Kosson et al., 2002). Interestingly however, although the high psychopathy group reported offending much earlier than the other groups, psychopathy scores were not associated with previous, index or future offending. Only the SAVRY risk ratings were found to be related with prior violent criminal offending $(r=.27)$ and violent recidivism $(r=.24)$. The PCL:YV factor 4 (antisocial behaviour), which encompasses items such as prior criminal behaviour, was found to be associated with violent recidivism $(r=.25)$. This outcome was also noted for the SAVRY historical subscale $(r=.24)$ which also contains items relating to past criminal behaviour.

In summary, psychopathy scores and risk of violence scores were found to be associated with other risk factors that have consistently been shown to be related antisocial behaviour in both adults and youth. The base rates of offending were as predicted and were consistent with previous findings. SAVRY risk scores were only related with prior violent offences and violent 
recidivism, which given the purpose of the instrument, this lends both concurrent and predictive validity for the ability of the test to distinguish violent behaviour amongst a youth sample who is in conflict with the law.

To achieve the second objective of this dissertation, the PCL:YV and the SAVRY were examined to determine if they would be able to predict future criminal convictions. As seen in the literature, the PCL:YV has been shown to be both predictive of non-violent and violent offending in youth (Corrado et al., 2004; Gretton et al., 2004; Schmidt et al., 2011; Stockdale et al., 2010; Vincent et al., 2003). However, other researchers were unable to replicate this finding (Cauffman et al., 2009; Dolan \& Rennie, 2008; Edens \& Cahill, 2007). Further, Dolan and Rennie (2008) have found that the SAVRY was moderately predictive of future general and violent offending, whereas Singh et al. (2011) found that the SAVRY had the highest predictive validity when violent offending was examined in comparison with nine other commonly used risk prediction tools. Further, Schmidt et al. (2011) found high AUCs on the SAVRY for both non-violent offending and violent offending amongst a sample of delinquent males who were referred by the court for a mental health assessment.

The results of the current study are somewhat mixed with what has previously been reported in the literature. The first set of analyses was to determine if the PCL:YV or the SAVRY could predict any recidivism one-year post release, five-years post release and ten-years post release. The results indicated that neither scale was able to significantly predict any recidivism, with the exception that the SAVRY risk rating was moderately predictive of general offending at 5-years post release (AUC $=.64)$. Vincent et al. (2011) found a moderate to large association with any recidivism after 1-2 years; however such associations were not replicated in the current study. 
Next, non-violent convictions were examined utilizing ROC procedures. The results revealed that again neither scale was able to adequately predict non-violent offending for any of the follow-up periods. The AUCs obtained in the current study were consistent with Douglas, Epstein, and Poythress (2008), Edens et al. (2007) and Dolan and Rennie (2008) for the PCL:YV and inconsistent with Dolan and Rennie (2008) and Schmidt et al. (2011) for the SAVRY. The results from the current study are suggestive that psychopathy measures may not be predictive of non-violent offending. This finding is not surprising as the scale was not designed as a criminal prediction tool, although it has come to be regarded as a useful utility in such regard, but rather, it was designed as a diagnostic tool for identifying psychopathic traits in youth. Further, the findings of the SAVRY are not surprising either as the function of the SAVRY is to assess the risk of violence amongst youth. Given the mixed results in the research community in regards to the usefulness of both scales in predicting non-violent offending, additional studies will have to determine whether such scales should be applied in such cases.

Cox survival regressions indicated that during the first year post release, both the PCL:YV and SAVRY risk scores were related to the imminence of non-violent offending. With every increase in PCL:YV and SAVRY risk score, youth had a 4\% increase in risk of nonviolent offending. The results were not replicable for the five-year and ten-year follow-up period, respectively. This indicates that the PCL:YV and SAVRY were only able to predict nonviolent offending for short term follow-up periods and not for long-term offending. This finding is consistent with Vincent et al. (2011) who found that the SAVRY was able to predict nonviolent recidivism at the 1-2 year follow-up range. When the youth were grouped into low, moderate and high scoring groups based upon their PCL:YV and SAVRY risk scores, the results indicated the PCL:YV groups did not differ on non-violent offending for any of the outcome 
periods, however the SAVRY groups differed on non-violent offending for both the 5-year and 10-year follow-up period. In both cases, the high scoring group recidivated more quickly than youth who scored in the low group. This suggests that SAVRY risk scores can distinguish high risk non-violent offending for longer periods post release. Again, this is similar to Vincent et al. (2011) who found that when SAVRY risk ratings were dichotomized into low versus moderate/high scoring groups, the moderate/high risk group had moderate associations with nonviolent offending 1-2 years after release.

When violent offending was examined, psychopathy scores were predictive of violent offending for offences committed during the first year post release, but not for 5 and 10-years post release. This is consistent with what Cauffman et al. (2009) indicated in their study that proximal predictiveness of the PCL:YV was better than longer-term predictiveness. As hypothesized, the only PCL:YV factor to be significantly predictive of violent offending was factor 4 (antisocial), however this factor was only significant for the first year post release. SAVRY risk, on the other hand, was not significantly predictive of short-term (one year post release) violent offending, but did show adequate predictive ability for violence occurring ten years post release (AUC of .67). This is consistent with Vincent et al.'s (2011) study which found that it often takes at least 5 years to be able to predict violent recidivism using the SAVRY. The SAVRY subscale of clinical features was moderately able to predict violent offending both at one-year and five-years post release however this subscale was unable to predict beyond five years.

When the imminence of offending was examined, several key results were obtained. Firstly, for violent offending during the first-year post release, the antisocial factor of the PCL:YV was related to timing of offending; with every unit increase in this factor, youth had a 
$7 \%$ increase risk in violent offending. No other score was related to offending for this outcome period. In examining five-years post release, several findings were noteworthy. First, total PCL:YV scores were related to the imminence of violent offending and with every unit increase in total PCL:YV score, youth had a $6 \%$ increase in risk of violently offending. When the factor scores were examined, again, factor 4 scores were indicative of imminence of offending, with youth having a $17 \%$ increase in risk with every until increase in factor 4 scores. SAVRY risk and historical scores were also indicative of violent offending during the five-year follow-up period, with both suggesting that higher scores on these measures was related to youth violently offending more quickly. Both scales were not related to the imminence of offending for the 10 year follow-up period. When the groups were divided into low, moderate and high scoring groups, the PCL:YV was able to distinguish between high and low scoring groups for one-year and five-year post release, but not for 10-years. Interestingly, the moderate scoring groups on the SAVRY risk scale differed from the low, but not the high scoring group, on violent offending for both 5 and 10-years after release. The high scoring SAVRY group did not differ from the low scoring group on any of the outcome periods.

In summary, contrary to what was hypothesized, neither the PCL:YV nor the SAVRY were predictive of non-violent offending. Timing of non-violent offences was found to be related to PCL:YV and SAVRY risk scores for short term follow-up but not for long-term follow-up. The finding that the PCL:YV was not predictive of non-violent convictions was consistent with Cauffman et al. (2009) and Edens and Cahill (2007) and is in line with what was reported by Douglas et al. (2008) in that the PCL:YV was not able to statistically predict nonviolent offending for both youth and adult offences. The PCL:YV and SAVRY performed as predicted for violent offending, however differences emerged on the timing of offences. The 
PCL:YV was a better predictor of recidivism during the year following release from custody, whereas the SAVRY was a better predictor of long-term violent persistence, similar to Vincent and colleagues (2011).

The mixed findings of past research demonstrate that there is wide variability in AUCs obtained for both non-violent and violent offending. This is not surprising given the differences in how outcome measures are defined, the ethnic and gender composition of samples and the length of follow-up periods examined. Further, some studies examine the predictiveness using retrospective designs, while others use prospective. This further adds to the complexity in understanding and compiling all the research findings to discern the generalizability of the findings.

The two instruments utilized in the current study compared reasonably well, despite the fact that they were designed for two separate purposes. The results may be in part due to the high degree of content-overlap and convergence, as indicated in the high correlation coefficients observed between the two measures. The PCL:YV was designed to be a diagnostic tool in the measure of psychopathic features amongst youth, whereas the SAVRY was developed as a structured professional judgment (SPJ) tool specifically for the risk of violence amongst youth. Although both are adequate tools in the prediction of future behaviour, as indicated in the findings, both show differing patterns of potential use. The PCL:YV appears to be better at examining proximal events, whereas the SAVRY may be more appropriate for long-term case planning. That speaks to the variety of potential uses such tools can be adapted, such as treatment planning and level of supervision (Olver et al., 2009). As noted by Olver and colleagues (2009), "a youth scoring high on a youth adaptation of the LSI, the PCL-YV, or the SAVRY is likely to pose substantial recidivism risk, general or violent. However, the different measures have 
clinical applications beyond mere recidivism prediction that may warrant consideration in their use. For instance, risk for violence may inform planning for violence-specific treatment, whereas a high level of psychopathic features may have important responsivity implications for programming and/or supervision" (p. 347).

Over the past few decades, interest into the youth psychopathy concept has focused on developing measures to study the symptoms of the disorder (Forth et al., 2003; Frick \& Hare, 2001; Lynam, 1996), the appropriateness of applying this concept with youth (Salekin \& Lochman, 2008), the temporal stability of this disorder (Frick et al., 2003) and the association between psychopathy and antisocial behaviour in adulthood (Gretton et al., 2004). However, it has only been in the past few years that an interest in protective factors as it relates to youth psychopathy has garnered much attention. Salekin and Lochman (2008) wrote that researchers should examine factors associated with the temporal stability of psychopathic features to identify factors that may be related to the manner in which youth develop prosocial ways and thus may be related to the desistence of criminal offending. If by identifying factors that protect against the stability of psychopathic features, it may help with possible intervention strategies that may lead to personality characteristics that ensure that persistent antisocial behaviour does not continue throughout the lifespan. This has implications for both the individual and society.

Many researchers have described the desistence of offending, particularly in youth (Piquero, Farrington, \& Blumstein, 2003) with few providing explanations as to why such behaviour declines over time (Laub \& Sampson, 2001). Most explanations of desistence appear to be focused on the developmental stage in which the adolescent person is undergoing, for example, changes within the individual, relationships, contexts and opportunities all influence the transition from adolescence into adulthood and serve to increase behaviours approved by 
society and decrease delinquent behaviour (Strouthamer-Loeber et al., 2004). Unfortunately at this point in time, we have no meta-analytic studies in which desistence is the focus. Further still is the paucity of research studies which examine the role of protective factors and how they relate to our understanding of risk assessments with young offenders.

Our understanding of protective factors is in its infancy stage. It is difficult to fully understand the complexity of the interaction between protective and risk factors and the strength of each separately and in conjunction with each other on subsequent behaviour. Moreover, for some the absence of a particular risk factor may serve as a protective factor (Strouthamer-Loeber et al., 2004). It is unclear what the true nature is of this relationship and only longitudinal studies which encompass regular measurement of both risk and protective factors across time will elucidate the true relationship between these factors. Our current understanding of protective factors in the desistence literature pertaining to youth offenders has largely been based on studies that have utilized the SAVRY. This scale uses factors that are deemed to be protective in a positive manner, meaning they are notable for their presence and are not simply the absence of a risk factor. However, given that our understanding of protective factors is limited, we must consider other factors that might serve as protective as we move forward with our quest to comprehend how protective factors can serve to increase juvenile criminal desistence.

Past studies have found that the protective factor of the SAVRY was predictive of nonoffending for general recidivism but not for violent offending (Dolan \& Rennie, 2008) and that the protective rating was negatively related to time to non-violent rearrest (Vincent et al., 2011) meaning that the more protective factors a youth had, the less likely he was to be rearrested. Lodewijks et al. (2010) found that youth who had just one or more SAVRY protective factors were less likely to commit a future criminal offence. 
The results of the current study were consistent with previous studies in that protective ratings were related with lower base rates of non-violent and violent offending (e.g., Lodewijks et al., 2010; Rennie \& Dolan, 2010). When the individual protective factors were examined, nonviolent desisters did not differ from persisters on whether an individual protective factor was present. Rennie and Dolan (2010) observed that youth who had each protective score present were less likely to commit non-violent offending, however their study did not examine whether youth desisted from non-violent offending. For violent offending, the current study found that desisters were more likely to have a positive attitude toward intervention and authority and were more likely to have a strong commitment to school. Rennie and Dolan (2010) found that youth with these protective factors had committed less violent offences 12 months post release. Upon further examination, it was noted that having a strong social supports and having a positive attitude toward intervention and authority meant that youth had fewer number of non-violent convictions post index release, again consistent with Rennie and Dolan (2010). Similar to Rennie and Dolan (2010), youth who had a strong commitment to school were found to have committed fewer violent offences during the entire follow-up period. When the PCL:YV and SAVRY risk scores were divided into low, moderate and high scoring groups, the high scoring group on both scales were found to have significantly fewer protective items present.

By utilizing logistic regressions, both the univariate and multivariate technique were employed. The univariate analysis examined each protective factor separately to discern if such factor was related to non-violent or violent offending. The results indicated that having a positive attitude toward intervention and authority was predictive of non-violent desistence. No other univariate factor indicated a relationship with non-violent desistence. When all factors were 
entered into the multivariate analysis, the effect of having a positive attitude toward intervention and authority was eliminated and no other factor proved to be related to non-violent desistence.

When univariate logistic regressions were examined for violent offending, several factors were found to be predictive of violent desistence, namely having strong social supports, having a positive attitude toward intervention and authority and having a strong commitment to school.

This is similar to the findings observed in Lodewijks et al.'s (2010) study. When the factors were entered at the multivariate level, only strong commitment to school remained significant. Interestingly, having pro-social involvement was found to be predictive of violent persistence. This finding has not been reported elsewhere in the literature and thus warrants further studies to examine whether such finding is an accurate reflection of violent offenders or whether it is an anomaly to the current study. It could be speculated that having pro-social involvement may be a protective factor with some youth offenders or even may serve as a protective factor with nonoffending youth, however with youth who engage in violent offending, other risk factors may be overshadowing the impact of having pro-social involvement. Rutter (1990) described a protective mechanism in which the addition of each protective factor in one's life reduces the impact of risk in negative outcomes. Since the current study found that most youth (70.3\%) had re-offended violently when they had either none or only one protective factor present, the impact of protective factors was negligible possibly as a result of having other, more prominent risk factors within their life.

The incremental validity of the protective score was examined to identify if adding such information to an assessment in conjunction with evaluations of risk, in this case psychopathy and risk of violence, would provide additional information that would assist with case management strategies. Overall, the results indicated that the prediction of non-violent offending 
was improved when protective ratings were added to the model when psychopathy was examined. Interestingly, when SAVRY risk and SAVRY protective were examined, the results indicated that each factor separately was predictive of persistence and desistence, respectively; however, when examined together, their combined influence was non-significant. When violent offending was examined, the effect was more pronounced. Adding protective information was able to account to almost $6 \%$ additional variance explained, over-and-above what was explained by psychopathy alone. Similarly, adding the protective factor to the model that included SAVRY risk added an additional variance explained, but at a lower level (2.3\%).

Researchers have examined additional psychosocial and cognitive factors that might serve as a protective mechanism in criminal offending. Stouthamer-Loeber and colleagues (2004) found that adolescents who did not receive harsh punishment by parents in early adolescence and who were employed or in school in early adulthood were more likely to desist in offending. Other studies have been unable to find any predictors of desistence in male juvenile offenders amongst a variety of psychosocial, physiological and cognitive variables (Loeber et al., 2007). Given this, our current understanding of the role protective factors play in criminal desistence may be better enhanced by making modifications to the SAVRY. Researchers have demonstrated that the protective items on the SAVRY buffer or mitigate the risk of violent offending (Lodewijks et al., 2010), however given that the items are scored on a dichotomous present or absent format, it may be advantageous to consider whether the items should be scored on a 3-point rating ranging from definitely does not apply, to somewhat applies, to definitely applies, which is akin to how risk factors are measured. This modification may help to explain additional variance in individuals who demonstrate some aspects of protective factors. This may help to maximize the ability of the SAVRY protective factor to predict future desistence. 
Lastly, the ROC analyses indicated that the ability of the protective score to adequately predict non-violent and violent desistence was, for the most part, contrary to what was hypothesized. The only significant prediction of desistence was for any recidivism during the 10-year follow-up period. Although the AUC for violent desistence was also significant at the 10-year follow-up time period, it was considered to be in the moderate range of accuracy (AUC $=.66)$.

The findings are suggestive that youth who have high numbers of protective features are less likely to have high numbers of non-violent and violent recidivism convictions. In line with other findings in the current study, non-violent persisters and desisters did not differ on the number of protective factors present, but violent persisters and desisters did. Youth who desisted in violent offending were more likely to have higher number of protective factors present. Particularly, having a strong commitment to school and having strong social supports were indicative of violent desistence.

Further, the results are suggestive of the importance of including protective factors in any assessment in which youth in conflict with the law are examined. The findings indicate that protective measures may buffer the effects of other risk factors, particularly psychopathy, which warrants further discussion and research. If protective factors are able to explain the amount of variance in a particular behaviour, even to a small degree, then we are obliged as researchers and clinicians to incorporate such information as a means of providing service delivery that will enhance the youth's quality of life and decrease his or her risk of a career of criminal offending.

\section{Study Implications}

There are several important implications that have come to light as a result of the findings of the current study. Firstly, the predictiveness of the PCL:YV for short and long term follow-up 
periods was counter to what other studies have demonstrated. Most notably, the findings of the current study in that the PCL:YV was not able to predict beyond one year for violent recidivism runs counter to a recent study by Schmidt et al. (2011). Schmidt et al.'s (2011) study is similar to the current study in that it examined the predictive validity of the PCL:YV and the SAVRY over a 10-year period and both were based on file information to score the PCL:YV and the SAVRY. Schmidt and colleagues found high predictiveness of the PCL:YV for non-violent offending $(\mathrm{AUC}=.78)$ and violent offending $(\mathrm{AUC}=.78)$ and demonstrated that the PCL:YV has the ability to predict recidivism over the long term. The authors of this study concluded that the PCL:YV is a meaningful predictor of recidivism and that those who scored high recidivated more quickly than those scoring in the low or moderate range. Given that the current study and the Schmidt et al. (2011) study are two of the few that have such long follow-up periods and that each of the two studies found conflicting results, this leads the author to consider two important implications as it pertains to male youth offenders.

Given the mixed results in the literature and what was found in the current study, we must be cautious of applying the PCL:YV to predict long-term recidivism. The inability of the current study to show long term predictiveness of re-offending among male youth offenders speaks to the need to re-examine the stability debate within this population. Past studies examining the stability of psychopathic traits has indicated that there is a modest link between child and adult psychopathy (Frick et al., 2003; Lynam et al., 2007). These studies suggest that some individuals who exhibit psychopathic traits in childhood grow up to be adult psychopathy but also, these studies highlight that a significant portion do not. Given that the current study was not able to demonstrate that criminal offending would be predictive by psychopathic traits assessed in adolescence beyond one year, this may be a reflection that amongst this age group, 
the stability of these traits is not as strong as once believed. Instability of psychopathic traits may be a reflection of differing trajectories in youth with factors allowing for change as well as maintenance of the disorder (Salekin, Rosenbaum, Lee, \& Lester, 2009). Researchers therefore must look to additional risk and protective factors as a means of describing what pathways certain youth are more likely to take.

The second implication pertains to labelling of youth as having psychopathic traits or being psychopathic. As indicated in the introduction, applying the label of psychopathy in youth results in more punitive decisions from probation officers, potential jurors, judges and clinicians (Boccaccini, Murrie, Clark, \& Cornell, 2008; Murrie, Cornell, \& McCoy, 2005; Rockett, Murrie, \& Boccaccini, 2007). These decisions carry significant outcomes, often in a negative manner. Given the stability of psychopathic traits in youth is questionable, applying psychopathic labels to youth who may outgrow these traits may have consequences beyond what the scale was intended to measure. Therefore, in the near future and until such debate regarding stability is more defined, the use of psychopathy assessments should only be considered for short-term decisions and not for any long-term decisions.

\section{Study Limitations}

A number of limitations of the present study are worth noting. Firstly, the use of official crime records data limits the ability to predict future behaviour with a high degree of accuracy. There is a known phenomenon in the criminology field known as the "dark figure" of crime (Coleman \& Moynihan, 1996). This refers to the underestimation of true criminal behaviour when only official records are examined. Further a limitation to the current study was that only criminal convictions were coded for outcome data. In some instances criminal charges were recorded on the official criminal record (CPIC) but given that this information was inconsistently 
recorded, it was omitted to ensure reliability in coding. Future studies may wish to improve upon this limitation by utilizing a more resource intensive follow-up by examining self-report and collateral report sources, such as prison records, parental reports and teacher ratings.

Another limitation to the present study pertains to the generalizability of the findings. The composition of the present study was with males who were predominantly Caucasian. Given the PCL:YV is widely used in applied and research settings, determining the generalizability, reliability and validity across different ethnicities is very important. Several studies in adult samples have shown little difference, if any, in various ethnic samples with male offenders (Cooke, Kosson, \& Michie, 2001; Skeem, Edens, Camp, \& Colwell, 2004; Sullivan, Abramowitz, Lopez, \& Kosson, 2006). In youth, McCoy and Edens (2006) also did not find any discernable difference between black and white youth offenders on PCL:YV scores.

Edens and Cahill (2007) have also found no differences between Caucasian, African American and Hispanic youth on psychopathy scores predicting recidivism. However, there appears to be evidence that the construct of psychopathy may not be measured in the same manner across different ethnic groups (McCoy \& Edens, 2006; Skeem et al., 2004). Edens et al. (2007) found in their meta-analysis that amongst ethnically diverse samples, a weaker relationship was noted between violence and recidivism than studies in which the samples were from primarily white participants. Future studies may wish to include a more ethnically diverse sample that it a better reflection of the Canadian population to more appropriately generalize the findings. Therefore, given the large proportion of Caucasian youth, the ability to fully gather information pertaining to the effects of ethnicity on the patterns of persistence and desistence was not possible. 
Further, given the sample consisted of only male youth, the ability to relate the findings of the current study to female youth are limited. There is controversy in regards to applying the psychopathic construct with females (Edens et al., 2001; Odgers, Reppucci, \& Moretti, 2005; Vincent, Odgers, McCormick, \& Corrado, 2008). Limiting the applicability of the results from the current study to only male youths does not add important information vis-à-vis female youth in conflict with the law. Future studies will need to expand the scope of predictive validity studies to ensure female youth are examined.

A third limitation of the current study was that it was an analysis of archival data. There are limits to the kind of information that can be gleaned from file information. The original purpose of research for each of the three cohorts was not related to the assessment of the risk of violence (via the SAVRY). Therefore, the SAVRY was retrospectively coded based on file and interview data that was collected on samples on which psychopathy was the main interest. Although the IRRs obtained for the SAVRY ratings were high, the internal consistencies of the SAVRY subscales were lower than what is typically reported in the literature (Borum et al., 2006). Low reliability may interfere with the predictive validity of the SAVRY, therefore future studies will need to be designed so that the SAVRY is coded at the time of the original assessment.

The results of the current study found that some subscales on the PCL:YV and SAVRY, as well as for conduct disorder, had low internal consistency, which has not been found in other studies (Borum et al, 2010). Typically, acceptable internal consistency is defined as coefficients greater than .70 . With this number set as the benchmark, the PCL:YV Factor 3 (behavioural), the SAVRY historical, social and protective, and conduct disorder symptoms all fell below this level. Having low internal consistency implies that the average inter-item correlation between 
the items comprising a subscale is low. When all items measure the same underlying construct, it is expected that a coefficient of one will be obtained. When values fall below one, this suggests that there is unshared variance or error entered into the measurement of the items.

Low internal consistency possibly implies that there is an invalid assessment or it could be the results of how the items are measured. For instance, Cronbach (1951) indicated that internal consistency is influenced by the number of items on which a scale is comprised. Further, Swailes and MacIntyre-Bhatty (2002) have suggested that internal consistency is difficult to measure when a scale has less than seven items. This was the case for Factor 3 of the PCL:YV, the SAVRY social and SAVRY protective factor. Cronbach (1951) offered a correction factor when scale length was small. This correction factor examines the mean interitem correction $(\rho)$ and is independent of scale length. By utilizing this formula, Factor 3 had a mean inter-item correlation of .23, the SAVRY social subscale had a mean inter-item correlation of .12, and the protective factor had a mean inter-item correlation of .26. Clark and Watson (2003) suggested that a $\rho$ within the range of .15 to .20 is acceptable. Utilizing this criteria, Factor 3 and the SAVRY protective factor were found to be in the acceptable range, however given that some of the subscales were below this threshold with or without the correction factor applied, future studies may wish to examine the findings to ensure that the results can be replicated. Until such time, results based on the subscales should be interpreted with caution.

Lastly, there were differences noted across the three samples. The 1986 sample consisted of youth who were in custody in British Columbia, whereas the 1994 and 2004 samples were comprised of youth from Ontario's facilities. It is unclear whether such differences produced a biasing effect. Additionally, the 2004 sample consisted of youth who were incarcerated as a result of the Youth Criminal Justice Act (YCJA), whereas the 1986 and 1994 samples contained 
youth who were incarcerated under the Young Offenders Act (YOA). The YCJA was enacted as a means of attempting to bring more emphasis on the protection of the public and to facilitate the detention of youth charged with offences under that Act or who pose a threat to society; therefore there may be differences in the types of offences for which the 2004 sample received custodial sentences. Further, the 2004 sample consisted of youth who were more likely to be on remand whereas the previous cohorts were predominately of youth serving custodial sentences. It is unclear whether these disparities impacted the current results. Future studies can overcome this issue by examining similar cohorts of youth.

\section{Future Directions}

There are several potential research studies that can arise from the findings and design of the current study. Firstly, it is imperative that we continue to examine the short and long-term predictive validity of both the SAVRY and the PCL:YV as the results of the current study and past research have indicated that there may be differences in the ability of each scale to predict proximal and distal events. Additional studies will be needed to apply these findings to females and to samples that do not consist of predominately Caucasian participants.

Previous studies (Stockdale et al., 2009) have examined the differences between youth who were assessed at a young age (12 to 15) and compared these findings to youth who were assessed at an older age (16 plus). Future studies would benefit from including such analyses in predictive validity studies. It is also warranted that given the findings that persisters differ from desisters on some social factors, additional information such as housing quality, community crime levels, anxiety, employment factors, type of substance use, etc., should be collected at time of original assessment to discern if other factors can distinguish between those who go on to offend in comparison with those who desist in their offending. Additional methods of 
assessment could also be employed to gather additional information, such as interviewing those who persist and desist as a means of improving our understanding of these two types of offenders. We also need to expand on our reliance on self-report measure to include as much collateral information as possible. The true nature of the predictive utility of the PCL:YV and the SAVRY on youth who desist or persist in offending will remain unknown in the absence of such research.

There is an extensive body of research literature pertaining to the onset and persistence of criminal offending (Farrington, 2007; Moffitt, 1993). Many studies have demonstrated that there is considerable consistency in conduct problems across the lifespan. It has been shown that individuals who engage in repeat offending were responsible for committing a large proportion of crimes and begin offending at an early age (Moffitt, 1993). Most researchers have focused on age of onset as a distinguishing feature of a trajectory towards a criminal career (Moffitt, 1993). It is important to also consider whether someone who has had several prior offences differs from individuals who have only one past offence to discern whether there are differing offending patterns during the follow-up period. Further, few studies have explored whether differences in types of offending impact this trajectory. Most research studies do not take into account the type of offence committed prior to release when examining types of offences that are committed post release. Often violent, non-violent and sexual offenders are combined into one category and followed to determine what type of offence was committed post release. As future studies are developed, we must move towards a more comprehensive approach to understanding juvenile criminal offending. It is important that we understand the trajectory of offending based upon the criminal behaviour in an offender's past. Are non-violent offenders more likely to recidivate than violent offenders? Without separating based on criminal histories, we are missing a more 
complete understanding of which types of offenders are more or less likely to recidivate. Future studies may wish to differentiate based on past criminal history to discern if such differences will be found.

\section{Conclusions}

The current study examined three main research questions: what factors associated with criminal persistence and desistence; is psychopathy and risk of violence able to predict future offending, and are protective factors related to the desistence of criminal offending. Overall, the present study suggests that the diagnosis of psychopathy is less related to the long term predictive ability of future criminal offending than the examination of the risk of violence in youth. The finding that the PCL:YV is not as predictive of future offending as the SAVRY risk ratings is consistent with previous studies. Other studies have indicated that the SAVRY offers the most predictive validity for youth and adult recidivism. Further, the AUCs for the SAVRY indicate that this instrument has an ability to predict violent offending, which is the intended purpose of the instrument.

A related point that should be considered pertains to specificity and sensitivity (Murphy \& Davidshofer, 2001). This implies that individuals who score high on psychopathy are likely to violently reoffend (high specificity), however those who violently reoffend may not score high on psychopathy (low sensitivity). The design of the SAVRY may be more appropriate to capture all violent offenders, whether or not they possess psychopathic traits, as it was designed to examine the risk of violent offending. Given this, it may be more appropriate to administer the SAVRY instead of the PCL:YV in forensic settings as a measure of risk. 


\section{Chapter V: References}

Alterman, A. I., Cacciola, J. S., \& Rutherford, M. J. (1993). Reliability of the revised psychopathy checklist in substance abuse patients. Psychological Assessment, 5, 442-448. doi:10.1037/1040-3590.5.4.442

American Psychiatric Association (1994). Diagnostic and statistical manual of mental disorders ( $4^{\text {th }}$ ed., revised). Washington, DC: American Psychiatric Association.

Andershed, H., Kerr, M., Stattin, H., \& Levander, S. (2002). Psychopathic traits in non-referred youths: A new assessment tool. In E. Blauuw \& L. Sheridan (Eds.), Psychopaths: Current international perspectives (pp. 131-158). The Hague: Elsevier.

Andrews, D.A, \& Bonta, J. (1995). The Level of Service Inventory-Revised. Toronto, ON: MultiHealth Systems.

Andrews, D. A., \& Bonta, J. (2010). The psychology of criminal conduct $\left(5^{\text {th }}\right.$ ed.). Cincinnati, $\mathrm{OH}$ : Anderson.

Andrews, D. A., Bonta, J., \& Wormith, J. S. (2004). Manual for the Level of Service/Case Management Inventory. Toronto, ON: Multi-Health Systems.

Barry, T. D., Barry, C. T., Deming, A. M., \& Lochman, J. E. (2008). Stability of psychopathic characteristics in childhood: The influence of social relationships. Criminal Justice and Behavior, 35, 244-262. doi:10.1177/0093854807310508

Bartol, C.R. (2002). Criminal behavior: A psychosocial approach, $6^{\text {th }}$ Ed. Upper Saddle River, NJ: Prentice Hall. 
Blackburn, R., \& Coid, J. W. (1998). Psychopathy and the dimensions of personality disorders in violent offenders. Personality and Individual Differences, 25, 129-145. doi:10.1016/S0191-8869(98)00027-0

Blair, J., Mitchell, D., \& Blair, K. (2005). The psychopath: Emotion and the brain. Malden: Blackwell Publishing. Retrieved from www.csa.com

Block, J. (1961). The Q-sort method in personality assessment and psychiatric research. Palo Alto, CA: Consulting Psychologists Press.

Blokland, A. A. J., \& Nieuwbeerta, P. (2005). The effects of life circumstances on longitudinal trajectories of offending. Criminology: An Interdisciplinary Journal, 43, 1203-1240. doi:10.1111/j.1745-9125.2005.00037.x

Boccaccini, M. T., Murrie, D. C., Clark, J. W., \& Cornell, D. G. (2008). Research report: Describing, diagnosing, and naming psychopathy: How do youth psychopathy labels influence jurors? Behavioral Sciences \& the Law, 26, 487-510. doi:10.1002/bs1.821

Borum, R. (2000). Assessing violence risk among youth. Journal of Clinical Psychology, 56, 1263-1288. doi:10.1002/1097-467956:101263:3.0.-D

Borum, R., Bartel, P., \& Forth, A.E. (2002). Manual for the Structured Assessment of Violence Risk in Youth (SAVRY): Version 1 Consultation Edition. Tampa, FL: University of South Florida.

Borum, R., Bartel, P., \& Forth, A.E. (2006). SAVRY: Structured assessment of violence risk in youth. Lutz, FL: Psychological Assessment Resources Inc. 
Borum, R., Lodewijks, H., Bartel, P. A., \& Forth, A. E. (2010). Structured assessment of violence risk in youth (SAVRY). New York, NY, US: Routledge/Taylor \& Francis Group. Retrieved from www.csa.com

Brandt, J. R., Kennedy, W. A., Patrick, C. J., \& Curtin, J. J. (1997). Assessment of psychopathy in a population of incarcerated adolescent offenders. Psychological Assessment, 9, 429435. doi:10.1037/1040-3590.9.4.429

Campbell, M. A., Porter, S., \& Santor, D. (2004). Psychopathic traits in adolescent offenders: An evaluation of criminal history, clinical, and psychosocial correlates. Behavioral Sciences \& the Law, 21, 23-47. doi:10.1002/bsl.572

Caprara, G. V., Barbaranelli, C., \& Pastorelli, C. (2001). Prosocial behavior and aggression in childhood and pre-adolescence. In A. C. Bohart, \& D. J. Stipek (Eds.), Constructive \& destructive behavior: Implications for family, school, \& society. (pp. 187-203). Washington, DC, US: American Psychological Association. doi:10.1037/10433-009

Catchpole, R. E. H., \& Gretton, H. M. (2003). The predictive validity of risk assessment with violent young offenders: A 1-year examination of criminal outcome. Criminal Justice and Behavior, 30, 688-708. doi:10.1177/0093854803256455

Cauffman, E., Kimonis, E. R., Dmitrieva, J., \& Monahan, K. C. (2009). A multimethod assessment of juvenile psychopathy: Comparing the predictive utility of the PCL:YV, YPI, and NEO PRI. Psychological Assessment, 21, 528-542. doi:10.1037/a0017367 
Chakhssi, F., de Ruiter, C., \& Bernstein, D. (2010). Change during forensic treatment in psychopathic versus nonpsychopathic offenders. Journal of Forensic Psychiatry \& Psychology, 21, 660-682. doi:10.1080/14789949.2010.483283

Chapman, J. F., Desai, R. A., Falzer, P. R., \& Borum, R. (2006). Violence risk and race in a sample of youth in juvenile detention: The potential to reduce disproportionate minority confinement. Youth Violence and Juvenile Justice, 4, 170-184.

doi:10.1177/1541204006286316

Cicchetti, D., \& Rogosch, F. A. (1996). Equifinality and multifinality in developmental psychopathology. Development and Psychopathology, 8, 597-600. doi:10.1017/S0954579400007318

Clark, L.A., \& Watson, D. (2003). Constructing validity: Basic issues in objective scale development. In A.E. Kazdin (Ed.). Methodological issues \& strategies in clinical research (3rd ed.) (pp. 207-231). Washington, DC, US: American Psychological Association.

Cleckley, H. (1976). The mask of sanity, $5^{\text {th }}$ ed. St. Louis: Mosby.

Coid, J. W. (2003). The co-morbidity of personality disorder and lifetime clinical syndromes in dangerous offenders. Journal of Forensic Psychiatry \& Psychology, 14, 341-366. doi:10.1080/1478994031000116381

Coid, J., Yang, M., Ullrich, S., Roberts, A., Moran, P., Bebbington, P., Brugha, T., Jenkins, R., Farrell, M., Lewis, G., Singleton, N., \& Hare, R. (2009). Gender differences in structured risk assessment: Comparing the accuracy of five instruments. Journal of Consulting and Clinical Psychology, 77, 337-348. doi:10.1037/a0015155 
Coie, J. D., Dodge, K. A., \& Kupersmidt, J. B. (1990). Peer group behavior and social status. In S. R. Asher, \& J. D. Coie (Eds.), Peer rejection in childhood. (pp. 17-59). New York, NY, US: Cambridge University Press. Retrieved from www.csa.com

Coleman, C., \& Moynihan, J. (1996). Understanding crime data: Haunted by the dark figure. Buckingham: Open University Press.

Cooke, D. J., Kosson, D. S., \& Michie, C. (2001). Psychopathy and ethnicity: Structural, item, and test generalizability of the Psychopathy Checklist—Revised (PCL-R) in Caucasian and African American participants. Psychological Assessment, 13, 531-542. doi:10.1037/10403590.13 .4 .531

Cooke, D. J., \& Michie, C. (2001). Refining the construct of psychopath: Towards a hierarchical model. Psychological Assessment, 13, 171-188. doi:10.1037/1040-3590.13.2.171

Cooke, D. J., Michie, C., \& Skeem, J. (2007). Understanding the structure of the psychopathy checklist-revised: An exploration of methodological confusion. British Journal of Psychiatry. Special Issue: Assessment, Risk and Outcome in Severe Personality Disorder, 190, s39-s50. doi:10.1192/bjp.190.5.s39

Cornell, D. G., Warren, J., Hawk, G., Stafford, E., Oram, G., \& Pine, D. (1996). Psychopathy in instrumental and reactive violent offenders. Journal of Consulting and Clinical Psychology, 64, 783-790. doi:10.1037/0022-006X.64.4.783

Corrado, R. R., Vincent, G. M., Hart, S. D., \& Cohen, I. M. (2004). Predictive validity of the Psychopathy Checklist: Youth Version for general and violent recidivism. Behavioral Sciences \& the Law, 22, 5-22. doi:10.1002/bsl.574 
Cronbach, L.J. (1951). Coefficient alpha and the internal structure of tests. Psychometrika, 16, 297-334. doi: 10.1007/BF02310555

Dolan, M. C., \& Rennie, C. E. (2008). The structured assessment of violence risk in youth as a predictor of recidivism in a United Kingdom cohort of adolescent offenders with conduct disorder. Psychological Assessment, 20, 35-46. doi:10.1037/1040-3590.20.1.35

Donnellan, M. B., Ge, X., \& Wenk, E. (2000). Cognitive abilities in adolescent-limited and lifecourse-persistent criminal offenders. Journal of Abnormal Psychology, 109, 396-402. doi:10.1037/0021-843X.109.3.396

Douglas, K. S., Cox, D. N., \& Webster, C. D. (1999). Violence risk assessment: Science and practice. Legal and Criminological Psychology, 4(Part 2), 149-184. doi:10.1348/135532599167824

Douglas, K. S., Epstein, M. E., \& Poythress, N. G. (2008). Criminal recidivism among juvenile offenders: Testing the incremental and predictive validity of three measures of psychopathic features. Law and Human Behavior, 32, 423-438. doi:10.1007/s10979-007-9114-8

Douglas, K. S., Ogloff, J. R. P., Nicholls, T. L., \& Grant, I. (1999). Assessing risk for violence among psychiatric patients: The HCR-20 violence risk assessment scheme and the Psychopathy Checklist: Screening Version. Journal of Consulting and Clinical Psychology, 67, 917-930. doi:10.1037/0022-006X.67.6.917 
D'Silva, K., Duggan, C., \& McCarthy, L. (2004). Does treatment really make psychopaths worse? A review of the evidence. Journal of Personality Disorders, 18, 163-177. doi:10.1521/pedi.18.2.163.32775

D'Unger, A. V., Land, K. C., \& McCall, P. L. (2002). Sex differences in age patterns of delinquent/criminal careers: Results from poisson latent class analyses of the Philadelphia cohort study. Journal of Quantitative Criminology, 18, 349-375. doi:10.1023/A:1021117626767

Edens, J. F., \& Cahill, M. A. (2007). Psychopathy in adolescence and criminal recidivism in young adulthood: Longitudinal results from a multiethnic sample of youthful offenders. Assessment, 14, 57-64. doi:10.1177/1073191106290711

Edens, J. F., Campbell, J. S., \& Weir, J. M. (2007). Youth psychopathy and criminal recidivism: A meta-analysis of the Psychopathy Checklist measures. Law and Human Behavior, 31 , 53-75. doi:10.1007/s10979-006-9019-y

Edens, J. F., Colwell, L. H., Desforges, D. M., \& Fernandez, K. (2005). The impact of mental health evidence on support for capital punishment: Are defendants labeled psychopathic considered more deserving of death? Behavioral Sciences \& the Law, 23, 603-625. doi:10.1002/bsl.660

Edens, J. F., Marcus, D. K., \& Vaughn, M. G. (2011). Exploring the taxometric status of psychopathy among youthful offenders: Is there a juvenile psychopath taxon? Law and Human Behavior, 35, 13-24. doi:10.1007/s10979-010-9230-8 
Edens, J. F., Marcus, D. K., Lilienfeld, S. O., \& Poythress, N. G., Jr. (2006). Psychopathic, not psychopath: Taxometric evidence for the dimensional structure of psychopathy. Journal of Abnormal Psychology, 115, 131-144. doi:10.1037/0021-843X.115.1.131

Edens, J. F., Skeem, J. L., Cruise, K. R., \& Cauffman, E. (2001). Assessment of "juvenile psychopathy" and its association with violence: A critical review. Behavioral Sciences \& the Law. Special Issue: Youth Violence, 19, 53-80. doi:10.1002/bs1.425

Farrington, D. (1983). Offending from 10 to 25 years of age. In K. Teilmann van Dusen \& S. Mednick (Eds.), Prospective studies in crime and delinquency (pp. 17-97). Boston: Kluwer-Nijhoff.

Farrington, D. P. (1989). Philadelphia cohort study produces offspring. US: American Psychological Association. doi:10.1037/031088

Farrington, D. P. (2005). The importance of child and adolescent psychopathy. Journal of Abnormal Child Psychology, 33, 489-497. doi:10.1007/s10802-005-5729-8

Farrington, D. P. (2007). Origins of violent behavior over the life span. New York, NY, US: Cambridge University Press. Retrieved from www.csa.com

Farrington, D.P., Coid, J.W., Harnett, L.M., Jolliffe, D., Soteriou, N., Turner, R.E., \& West, D.J. (2006). Criminal careers up to age 50 and life success up to age 48: New findings from the Cambridge study in delinquent development $\left(2^{\text {nd }}\right.$ ed.). London, UK: Home Office.

Flight, J. I., \& Forth, A. E. (2007). Instrumentally violent youths: The roles of psychopathic traits, empathy, and attachment. Criminal Justice and Behavior, 34, 739-751. doi:10.1177/0093854807299462 
Forth, A.E. (1995). Psychopathy and young offenders: Prevalence, family background, and violence. Program Branch Users Report. Ottawa, ON: Minister of the Solicitor General of Canada.

Forth, A. E., \& Book, A. S. (2007). Psychopathy in youth: A valid construct? In H. Hervé, \& J. C. Yuille (Eds.), The psychopath: Theory, research, and practice. (pp. 369-387). Mahwah, NJ, US: Lawrence Erlbaum Associates Publishers. Retrieved from whw.csa.com

Forth, A. E., \& Book, A. S. (2010). Psychopathic traits in children and adolescents: The relationship with antisocial behaviors and aggression. In R.T. Salekin, \& D.R. Lynam (Eds.) Handbook of child and adolescent psychopathy (pp. 251-283). New York, NY, US: Guilford Press. Retrieved from www.csa.com

Forth, A.E., \& Burke, H.C. (1998). Psychopathy in adolescence: Assessment, violence, and developmental precursors. In D.J. Cooke, A.E. Forth, \& R.D. Hare (Eds.), Psychopathy: Theory, research, and implications for society (pp. 205-229). Norwell, MA: Kluwer Academic Publishers.

Forth, A. E., Hart, S. D., \& Hare, R. D. (1990). Assessment of psychopathy in male young offenders. Psychological Assessment: A Journal of Consulting and Clinical Psychology, 2, 342-344. doi:10.1037/1040-3590.2.3.342

Forth, A.E., Kosson, D. \& Hare, R.D. (2003). The Hare Psychopathy Checklist: Youth Version. Toronto: Multi-Health Systems. 
Forth, A. E., \& Mailloux, D. L. (2000). Psychopathy in youth: What do we know? In C. B. Gacono (Ed.), The clinical and forensic assessment of psychopathy: A practitioner's guide. (pp. 25-54). Mahwah, NJ, US: Lawrence Erlbaum Associates Publishers. Retrieved from www.csa.com

Frick, P. J. (2000). The problems of internal validation without a theoretical context: The different conceptual underpinnings of psychopathy and the disruptive behavior disorder criteria. Psychological Assessment, 12, 451-456. doi:10.1037/1040-3590.12.4.451

Frick, P.J., \& Hare, R.D. (2001). Antisocial Process Screening Device. Toronto, ON: MultiHealth Systems.

Frick, P. J., Kimonis, E. R., Dandreaux, D. M., \& Farell, J. M. (2003). The 4 year stability of psychopathic traits in non-referred youth. Behavioral Sciences \& the Law, 21, 713-736. doi: $10.1002 / b s l .568$

Frick, P. J., O'Brien, B. S., Wootton, J. M., \& McBurnett, K. (1994). Psychopathy and conduct problems in children. Journal of Abnormal Psychology, 103, 700-707. doi:10.1037/0021843X.103.4.700

Fritz, M. V., Wiklund, G., Koposov, R. A., af Klinteberg, B., \& Ruchkin, V. V. (2008). Psychopathy and violence in juvenile delinquents: What are the associated factors? International Journal of Law and Psychiatry. Special Issue: Psychopathic Traits and Risk Assessment in Children and Adolescents, 31, 272-279. doi:10.1016/j.ijlp.2008.04.010

Gammelgård, M., Koivisto, A., Eronen, M., \& Kaltiala-Heino, R. (2008). The predictive validity of the structured assessment of violence risk in youth (SAVRY) among institutionalized 
adolescents. Journal of Forensic Psychiatry \& Psychology, 19, 352-370. doi:10.1080/14789940802114475

Glueck, S., \& Glueck, E. (1940). Juvenile delinquents grown up. Oxford, England: Commonwealth Fund. Retrieved from www.csa.com

Gottfredson, M. R., \& Hirschi, T. (1990). A general theory of crime Stanford University Press. Retrieved from www.csa.com

Gretton, H. M., Hare, R. D., \& Catchpole, R. E. H. (2004). Psychopathy and offending from adolescence to adulthood: A 10-year follow-up. Journal of Consulting and Clinical Psychology, 72, 636-645. doi:10.1037/0022-006X.72.4.636

Grove, W. M., \& Meehl, P. E. (1996). Comparative efficiency of informal (subjective, impressionistic) and formal (mechanical, algorithmic) prediction procedures: The clinical-statistical controversy. Psychology, Public Policy, and Law, 2, 293-323. doi:10.1037/1076-8971.2.2.293

Hare, R. D. (1980). A research scale for the assessment of psychopathy in criminal populations. Personality and Individual Differences, 1, 111-119. doi:10.1016/0191-8869(80)90028-8

Hare, R. D. (1991). The Hare Psychopathy Checklist-Revised. Toronto: Multi-Health

Hare, R. D. (1993). Without conscience: The disturbing world of the psychopaths among us. New York: Simon \& Schuster.

Hare, R. D. (1996). Psychopathy: A clinical construct whose time has come. Criminal Justice and Behavior, 23, 25-54. doi:10.1177/0093854896023001004 
Hare, R. D. (2003). Hare PCL-R TM. 2nd Ed. Technical Manual. Toronto: Multi-Health Systems, Inc.

Hare, R. D., Clark, D., Grann, M., \& Thornton, D. (2000). Psychopathy and the predictive validity of the PCL-R: An international perspective. Behavioral Sciences \& the Law. Special Issue: International Perspectives on Psychopathic Disorders, 18, 623-645. doi:10.1002/1099-0798(200010)18:5<623::AID-BSL409>3.0.CO;2-W

Hare, R. D., Forth, A. E., \& Strachan, K. E. (1992). Psychopathy and crime across the life span. In R. D. Peters, R. J. McMahon \& V. L. Quinsey (Eds.), Aggression and violence throughout the life span (pp. 285-300). Thousand Oaks, CA, US: Sage Publications, Inc. Retrieved from www.csa.com

Hare, R. D., McPherson, L. M., \& Forth, A. E. (1988). Male psychopaths and their criminal careers. Journal of Consulting and Clinical Psychology, 56, 710-714. doi:10.1037/0022006X.56.5.710

Harris, G. T., Rice, M. E., \& Quinsey, V. L. (1994). Psychopathy as a taxon: Evidence that psychopaths are a discrete class. Journal of Consulting and Clinical Psychology, 62, 387397. doi:10.1037/0022-006X.62.2.387

Hart, J. L., O'Toole, S. K., Price-Sharps, J. L., \& Shaffer, T. W. (2007). The risk and protective factors of violent juvenile offending: An examination of gender differences. Youth Violence and Juvenile Justice, 5, 367-384. doi:10.1177/1541204006297367

Hart, S. D., Cox, D. N., \& Hare, R. D. (1995). Manual for the Hare Psychopathy Checklist: Screening Version (PCL: SV). Toronto, Canada: Multi-Health Systems. 
Hart, S. D., \& Dempster, R. J. (1997). Impulsivity and psychopathy. In C. D. Webster, \& M. A. Jackson (Eds.), Impulsivity: Theory, assessment, and treatment (pp. 212-232). New York, NY, US: Guilford Press. Retrieved from www.csa.com

Hart, S. D., Watt, K. A., \& Vincent, G. M. (2002). Commentary on Seagrave and Grisso: Impressions of the state of the art. Law and Human Behavior, 26, 241-245. doi:10.1023/A:1014648227688

Hemphill, J. F., Hare, R. D., \& Wong, S. (1998). Psychopathy and recidivism: A review. Legal and Criminological Psychology, 3(Part1), 139-170. Retrieved from www.csa.com

Herpertz, S. C., \& Sass, H. (2000). Emotional deficiency and psychopathy. Behavioral Sciences \& the Law. Special Issue: International Perspectives on Psychopathic Disorders, 18, 567-580. doi:10.1002/1099-0798(200010)18:5<567::AID-BSL410>3.0.CO;2-8

Hervé, H. (2007). Psychopathy across the ages: A history of the hare psychopath. In H. Hervé, \& J. C. Yuille (Eds.), The psychopath: Theory, research, and practice (pp. 31-55). Mahwah, NJ, US: Lawrence Erlbaum Associates Publishers. Retrieved from www.csa.com

Hinshaw, S. P. (1992). Externalizing behavior problems and academic underachievement in childhood and adolescence: Causal relationships and underlying mechanisms. Psychological Bulletin, 111, 127-155. doi:10.1037/0033-2909.111.1.127

Hinshaw, S. P., \& Lee, S. S. (2003). Conduct and oppositional defiant disorders. In E. J. Mash, \& R. A. Barkley (Eds.), Child psychopathology (2nd ed) (pp. 144-198). New York, NY, US: Guilford Press. Retrieved from www.csa.com 
Hoge, R. D., Andrews, D. A., \& Leschied, A. W. (1996). An investigation of risk and protective factors in a sample of youthful offenders. Journal of Child Psychology and Psychiatry, 37, 419-424. doi:10.1111/j.1469-7610.1996.tb01422.x

Horney, J., Osgood, D. W., \& Marshall, I. H. (1995). Criminal careers in the short-term: Intraindividual variability in crime and its relation to local life circumstances. American Sociological Review, 60, 655-673. doi:10.2307/2096316

Howell, J.C. (2003). Preventing and reducing juvenile delinquency: A comprehensive framework. Thousand Oaks, CA: Sage.

Johnstone, L., \& Cooke, D.J. (2004). Empathy and offending: A systematic review and metaanalysis. Aggression and Violent Behavior, 9, 441-476.

Jones, S., \& Cauffman, E. (2008). Juvenile psychopathy and judicial decision making: An empirical analysis of an ethical dilemma. Behavioral Sciences \& the Law, 26, 151-165. doi:10.1002/bsl.792

Jones, S., Cauffman, E., Miller, J. D., \& Mulvey, E. (2006). Investigating different factor structures of the psychopathy checklist: Youth version: Confirmatory factor analytic findings. Psychological Assessment, 18, 33-48. doi:10.1037/1040-3590.18.1.33

Kimonis, E. R., Frick, P. J., \& Barry, C. T. (2004). Callous-unemotional traits and delinquent peer affiliation. Journal of Consulting and Clinical Psychology, 72, 956-966. doi:10.1037/0022-006X.72.6.956

Kosson, D. S., Cyterski, T. D., Steuerwald, B. L., Neumann, C. S., \& Walker-Matthews, S. (2002). The reliability and validity of the psychopathy checklist: Youth version 
(PCL:YV) in non-incarcerated adolescent males. Psychological Assessment, 14, 97-109. doi:10.1037/1040-3590.14.1.97

Larsson, H., Andershed, H., \& Lichtenstein, P. (2006). A genetic factor explains most of the variation in the psychopathic personality. Journal of Abnormal Psychology, 115, 221230. doi:10.1037/0021-843X.115.2.221

Laub, J. H., \& Sampson, R. J. (2003). Shared beginnings, divergent lives: Delinquent boys to age 70. Cambridge, MA, US: Harvard University Press. Retrieved from www.csa.com

Laursen, B., Finkelstein, B. D., \& Townsend Betts, N. (2001). A developmental meta-analysis of peer conflict resolution. Developmental Review, 21, 423-449. doi:10.1006/drev.2000.0531

Lee, Z., Klaver, J. R., Hart, S. D., Moretti, M. M., \& Douglas, K. S. (2009). Short-term stability of psychopathic traits in adolescent offenders. Journal of Clinical Child and Adolescent Psychology, 38, 595-605. doi:10.1080/15374410903103536

Leistico, A. R., Salekin, R. T., DeCoster, J., \& Rogers, R. (2008). A large-scale meta-analysis relating the hare measures of psychopathy to antisocial conduct. Law and Human Behavior, 32, 28-45. doi:10.1007/s10979-007-9096-6

Lilienfeld, S. O. (1998). Methodological advances and developments in the assessment of psychopathy. Behaviour Research and Therapy, 36, 99-125. doi:10.1016/S00057967(97)10021-3 
Lilienfeld, S. O., \& Andrews, B. P. (1996). Development and preliminary validation of a selfreport measure of psychopathic personality traits in noncriminal populations. Journal of Personality Assessment, 66, 488-524.doi:10.1207/s15327752jpa6603_3

Lloyd, C. (2007). Perceptions of self and desistance: Investigating positive attributes associated with exiting crime. Unpublished master's thesis. Carleton University, Ottawa, Ontario, Canada.

Lochman, J. E., \& Wayland, K. K. (1994). Aggression, social acceptance, and race as predictors of negative adolescent outcomes. Journal of the American Academy of Child \& Adolescent Psychiatry, 33, 1026-1035. doi:10.1097/00004583-199409000-00014

Lodewijks, H. P. B., de Ruiter, C., \& Doreleijers, T. A. H. (2010). The impact of protective factors in desistance from violent reoffending: A study in three samples of adolescent offenders. Journal of Interpersonal Violence, 25, 568-587.

doi:10.1177/0886260509334403

Loeber, R. (1996). Developmental continuity, change, and pathways in male juvenile problem behaviors and delinquency. In J. D. Hawkins (Ed.), Delinquency and crime: Current theories (pp. 1-27). New York, NY, US: Cambridge University Press. Retrieved from www.csa.com

Loeber, R., \& Farrington, D. P. (2000). Young children who commit crime: Epidemiology, developmental origins, risk factors, early interventions, and policy implications. Development and Psychopathology, 12, 737-762. doi:10.1017/S0954579400004107 
Loeber, R., Brinthaupt, V. P., \& Green, S. M. (1990). Attention deficits, impulsivity, and hyperactivity with or without conduct problems: Relationships to delinquency and unique contextual factors. In R. J. McMahon, \& R. D. Peters (Eds.), Behavior disorders of adolescence: Research, intervention, and policy in clinical and school settings (pp. 3961). New York, NY, US: Plenum Press. Retrieved from www.csa.com

Loeber, R., Pardini, D. A., Stouthamer-Loeber, M., \& Raine, A. (2007). Do cognitive, physiological, and psychosocial risk and promotive factors predict desistance from delinquency in males? Development and Psychopathology, 19, 867-887. doi:10.1017/S0954579407000429

Loney, B. R., Taylor, J., Butler, M., \& Iacono, W. G. (2002). The Minnesota Temperament Inventory: A psychometric study of adolescent self-reported psychopathy. Unpublished manuscript.

Loney, B. R., Taylor, J., Butler, M. A., \& Iacono, W. G. (2007). Adolescent psychopathy features: 6-year temporal stability and the prediction of externalizing symptoms during the transition to adulthood. Aggressive Behavior, 33, 242-252. doi:10.1002/ab.20184

Lynam, D. R. (1996). Early identification of chronic offenders: Who is the fledgling psychopath? Psychological Bulletin, 120, 209-234. doi:10.1037/0033-2909.120.2.209

Lynam, D. R. (1997). Pursuing the psychopath: Capturing the fledgling psychopath in a nomological net. Journal of Abnormal Psychology, 106, 425-438. doi:10.1037/0021843X.106.3.425 
Lynam, D. R. (1998). Early identification of the fledgling psychopath: Locating the psychopathic child in the current nomenclature. Journal of Abnormal Psychology, 107, 566-575. doi:10.1037/0021-843X.107.4.566

Lynam, D. R. (2002). Fledgling psychopathy: A view from personality theory. Law and Human Behavior, 26, 255-259. doi:10.1023/A:1014652328596

Lynam, D. R., Caspi, A., Moffitt, T. E., Loeber, R., \& Stouthamer-Loeber, M. (2007). Longitudinal evidence that psychopathy scores in early adolescence predict adult psychopathy. Journal of Abnormal Psychology, 116, 155-165. doi:10.1037/0021$843 X .116 .1 .155$

Lynam, D. R., \& Gudonis, L. (2005). The development of psychopathy. Annual Review of Clinical Psychology, 1, 381-407. doi:10.1146/annurev.clinpsy.1.102803.144019

Lynam, D., Moffitt, T. E., \& Stouthamer-Loeber, M. (1993). Explaining the relation between IQ and delinquency: Class, race, test motivation, school failure, or self-control? Journal of Abnormal Psychology, 102, 187-196. doi:10.1037/0021-843X.102.2.187

Lynam, D. R., \& Widiger, T. A. (2007). Using a general model of personality to identify the basic elements of psychopathy. Journal of Personality Disorders, 21, 160-178. doi:10.1521/pedi.2007.21.2.160

Lyon, D. R., \& Ogloff, J. R. P. (2000). Legal and ethical issues in psychopathy assessment. In C. B. Gacono (Ed.), The clinical and forensic assessment of psychopathy: A practitioner's guide (pp. 139-173). Mahwah, NJ, US: Lawrence Erlbaum Associates Publishers. Retrieved from www.csa.com 
Maquin, E., \& Loeber, R. (1996). Academic performance and delinquency. In M. Tonry (Ed.), Crime and justice: A review of research, Vol. 220 (pp.145-264). Chicago: University of Chicago Press.

Marcus, D. K., John, S. L., \& Edens, J. F. (2004). A taxometric analysis of psychopathic personality. Journal of Abnormal Psychology, 113, 626-635. doi:10.1037/0021843X.113.4.626

Marshall, L. A., \& Cooke, D. J. (1999). The childhood experiences of psychopaths: A retrospective study of familial and societal factors. Journal of Personality Disorders, 13, 211-225. Retrieved from www.csa.com

Masten, A. S. (2001). Ordinary magic: Resilience processes in development. American Psychologist, 56, 227-238. doi:10.1037/0003-066X.56.3.227

McCord, W., \& McCord, J. (1964). The psychopath: An essay on the criminal mind. Oxford, England: D. Van Nostrand. Retrieved from www.csa.com

McCoy, W. K., \& Edens, J. F. (2006). Do black and white youths differ in levels of psychopathic traits? A meta-analysis of the psychopathy checklist measures. Journal of Consulting and Clinical Psychology, 74, 386-392. doi:10.1037/0022-006X.74.2.386

McDermott, P. A., Alterman, A. I., Cacciola, J. S., Rutherford, M. J., Newman, J. P., \& Mulholland, E. M. (2000). Generality of psychopathy Checklist—Revised factors over prisoners and substance-dependent patients. Journal of Consulting and Clinical Psychology, 68, 181-186. doi:10.1037/0022-006X.68.1.181 
McGraw, K. O., \& Wong, S. P. (1996). Forming inferences about some intraclass correlation coefficients. Psychological Methods, 1, 30-46. doi:10.1037/1082-989X.1.1.30

Messick, S. (1995). Validity of psychological assessment: Validation of inferences from persons' responses and performances as scientific inquiry into score meaning. American Psychologist, 50, 741-749. doi:10.1037/0003-066X.50.9.741

Moffitt, T. E. (1993). Adolescence-limited and life-course-persistent antisocial behavior: A developmental taxonomy. Psychological Review, 100, 674-701. doi:10.1037/0033295X.100.4.674

Moffitt, T. E. (2005). The new look of behavioral genetics in developmental psychopathology: Gene-environment interplay in antisocial behaviors. Psychological Bulletin, 131, 533554. doi:10.1037/0033-2909.131.4.533

Moffitt, T.E., Caspi, A., Dickson, N., Silva, P., \& Stanton, W. (1996). Childhood-onset versus adolescent-onset antisocial conduct problems in males: Natural history from ages 3 to 18 years. Development and Psychopathology, 8, 399-424.

Muñoz, L. C., Kerr, M., \& Bešić, N. (2008). The peer relationships of youths with psychopathic personality traits: A matter of perspective. Criminal Justice and Behavior, 35, 212-227. doi:10.1177/0093854807310159

Murrie, D. C., Cornell, D. G., \& McCoy, W. K. (2005). Psychopathy, conduct disorder, and stigma: Does diagnostic labeling influence juvenile probation officer recommendations? Law and Human Behavior, 29, 323-342. doi:10.1007/s10979-005-2415-x 
Murrie, D. C., Cornell, D. G., Kaplan, S., McConville, D., \& Levy-Elkon, A. (2004).

Psychopathy scores and violence among juvenile offenders: A multi-measure study. Behavioral Sciences \& the Law, 22, 49-67. doi:10.1002/bsl.573

Murrie, D. C., Marcus, D. K., Douglas, K. S., Lee, Z., Salekin, R. T., \& Vincent, G. (2007). Youth with psychopathy features are not a discrete class: A taxometric analysis. Journal of Child Psychology and Psychiatry, 48, 714-723. doi:10.1111/j.1469-7610.2007.01734.x

National Research Council, Committee on Risk Perception and Communication, Commission on Behavioral and Social Sciences and Education, Commission on Physical Sciences, Mathematics, and Resources. (1989). Improving risk communication. Washington, DC: National Press Academy.

Nagin, D.S., Farrington, D.P., \& Moffitt, T.E. (1995). Life-course trajectories of different types of offenders. Criminology, 33, 111-139. DOI: 10.1111/j.1745-9125.1995.tb01173.x

Nagin, D. S., and Land, K. C. (1993). Age, criminal careers, and population heterogeneity: of delinquent/criminal careers? Results from mixed poisson regression analyses. American Journal of Sociology, 103, 1593-1630.

Neumann, C. S., Kosson, D. S., Forth, A. E., \& Hare, R. D. (2006). Factor structure of the hare psychopathy checklist: Youth version (PCL: YV) in incarcerated adolescents. Psychological Assessment, 18, 142-154. doi:10.1037/1040-3590.18.2.142

Odgers, C. L., Reppucci, N. D., \& Moretti, M. M. (2005). Nipping psychopathy in the bud: An examination of the convergent, predictive, and theoretical utility of the PCL-YV among adolescent girls. Behavioral Sciences \& the Law, 23, 743-763. doi:10.1002/bsl.664 
Ogloff, J. R., Wong, S., \& Greenwood, A. (1990). Treating criminal psychopaths in a therapeutic community program. Behavioral Sciences \& the Law, 8, 181-190.

doi:10.1002/bsl.2370080210

Ollendick, T. H., \& Vasey, M. W. (1999). Developmental theory and the practice of clinical child psychology. Journal of Clinical Child Psychology, 28, 457-466. doi:10.1207/S15374424JCCP2804_4

Olver, M. E., Stockdale, K. C., \& Wormith, J. S. (2009). Risk assessment with young offenders: A meta-analysis of three assessment measures. Criminal Justice and Behavior, 36, 329353. doi: $10.1177 / 0093854809331457$

Olver, M. E., \& Wong, S. C. P. (2009). Therapeutic responses of psychopathic sexual offenders: Treatment attrition, therapeutic change, and long-term recidivism. Journal of Consulting and Clinical Psychology, 77, 328-336. doi:10.1037/a0015001

O’Neil, M.L., Lidz, V., \& Heilburn, K. (2003). Predictors and correlates of psychopathic characteristics in substance abusing adolescents. International Journal of Forensic Mental Health, 2, 35-45.

Pardini, D. A., Barry, T. D., Barth, J. M., Lochman, J. E., \& Wells, K. C. (2006). Self-perceived social acceptance and peer social standing in children with aggressive-disruptive behaviors. Social Development, 15, 46-64. doi:10.1111/j.1467-9507.2006.00329.x

Paternoster, R., Dean, C. W., Piquero, A., Mazerolle, P., \& Brame, R. (1997). Generality, continuity, and change in offending. Journal of Quantitative Criminology, 13, 231-266. doi:10.1007/BF02221092 
Patrick, C. J., \& Zempolich, K. A. (1998). Emotion and aggression in the psychopathic personality. Aggression and Violent Behavior, 3, 303-338. doi:10.1016/S13591789(97)00003-7

Penney, S. R., Lee, Z., \& Moretti, M. M. (2010). Gender differences in risk factors for violence: An examination of the predictive validity of the structured assessment of violence risk in youth. Aggressive Behavior, 36, 390-404. doi:10.1002/ab.20352

Penney, S. R., \& Moretti, M. M. (2005). The transfer of juveniles to adult court in Canada and the United States: Confused agendas and compromised assessment procedures. The International Journal of Forensic Mental Health, 4, 19-37. Retrieved from www.csa.com

Pennington, L.A. (1966). Psychopathic and criminal behaviour. In L.A. Pennington \& I.A. Berg (Eds.), An introduction to clinical psychology. New York: Ronald Press.

Piatigorsky, A., \& Hinshaw, S. P. (2004). Psychopathic traits in boys with and without attentionDeficit/Hyperactivity disorder: Concurrent and longitudinal correlates. Journal of Abnormal Child Psychology: An Official Publication of the International Society for Research in Child and Adolescent Psychopathology, 32, 535-550. doi:10.1023/B:JACP.0000037782.28482.6b

Piquero, A. R., Farrington, D. P., \& Blumstein, A. (2003). The criminal career paradigm. In M. Tonry (Ed.), Crime and justice: An annual review of research (Vol. 27, pp. 137-283). Chicago, Il: University of Chicago Press.

Piquero, A. R., Farrington, D. P., Nagin, D. S., \& Moffitt, T. E. (2010). Trajectories of offending and their relation to life failure in late middle age: Findings from the Cambridge study in 
delinquent development. Journal of Research in Crime and Delinquency, 47, 151-173. doi: $10.1177 / 0022427809357713$

Porter, S., Birt, A., \& Boer, D. P. (2001). Investigation of the criminal and conditional release profiles of Canadian federal offenders as a function of psychopathy and age. Law and Human Behavior, 25, 647-661. doi:10.1023/A:1012710424821

Quinsey, V. L., Harris, G. T., Rice, M. E., \& Cormier, C. A. (2006). Violent offenders: Appraising and managing risk (2nd ed.). Washington, DC: American Psychological Association.

Rennie, C. E., \& Dolan, M. C. (2010). The significance of protective factors in the assessment of risk. Criminal Behaviour and Mental Health, 20, 8-22. doi:10.1002/cbm.750

Rice, M.E., \& Harris, G.T. (2005). Comparing effect sizes in follow-up studies: ROC area, Cohen's $d$ and $r$. Law and Human Behavior, 29, 615-629. doi: 10.1007/s10979-005$6832-7$

Rice, M. E., Harris, G. T., \& Cormier, C. A. (1992). An evaluation of a maximum security therapeutic community for psychopaths and other mentally disordered offenders. Law and Human Behavior, 16, 399-412. doi:10.1007/BF02352266

Rockett, J. L., Murrie, D. C., \& Boccaccini, M. T. (2007). Diagnostic labeling in juvenile justice settings: Do psychopathy and conduct disorder findings influence clinicians? Psychological Services, 4, 107-122. doi:10.1037/1541-1559.4.2.107 
Rotenberg, M., \& Diamond, B. L. (1971). The biblical conception of psychopathy: The law of the stubborn and rebellious son. Journal of the History of the Behavioral Sciences, 7, 2938. doi:10.1002/1520-6696(197101)7:1<29::AID-JHBS2300070105>3.0.CO;2-A

Rutherford, M. J., Cacciola, J. S., Alterman, A. I., \& McKay, J. R. (1996). Reliability and validity of the revised psychopathy checklist in women methadone patients. Assessment, 3, 145-156. Retrieved from www.csa.com

Rutter, M. (1990). Psychosocial resilience and protective mechanisms. In J. Rolf, A. Masten, D. Cichetti, K. Nuechterlein, \& S. Weintraub (Eds.). Risk and protective factors in the development of psychopathology (pp. 181-214). Cambridge, UK: Cambridge University Press.

Rutter, M., Giller, H., \& Hagell, A. (1998). Antisocial behavior by young people. New York, NY, US: Cambridge University Press. Retrieved from www.csa.com

Rygaard, N. P. (1998). Psychopathic children: Indicators of organic dysfunction. In T. Millon, E. Simonsen, M. Birket-Smith \& R. D. Davis (Eds.), Psychopathy: Antisocial, criminal, and violent behavior (pp. 247-259). New York, NY, US: Guilford Press. Retrieved from $\underline{\text { www.csa.com }}$

Salekin, R. T., Brannen, D. N., Zalot, A. A., Leistico, A., \& Neumann, C. S. (2006). Factor structure of psychopathy in youth: Testing the applicability of the new four-factor model. Criminal Justice and Behavior, 33, 135-157. doi:10.1177/0093854805284416 
Salekin, R. T., \& Lochman, J. E. (2008). Child and adolescent psychopathy: The search for protective factors. Criminal Justice and Behavior, 35, 159-172. doi: $10.1177 / 0093854807311330$

Salekin, R. T., Rogers, R., \& Machin, D. (2001). Psychopathy in youth: Pursuing diagnostic clarity. Journal of Youth and Adolescence, 30, 173-195. doi:10.1023/A:1010393708227

Salekin, R.T., Rosenbaum, J., Lee, Z., \& Lester, W.S. (2009). Child and adolescent psychopathy: Like a painting by Monet. Youth Violence and Juvenile Justice, 7, 239255. doi: $10.1177 / 1541204009333832$

Saltaris, C. (2002). Psychopathy in juvenile offenders: Can temperament and attachment be considered as robust developmental precursors? Clinical Psychology Review, 22, 729752. doi:10.1016/S0272-7358(01)00122-2

Sampson, R. J., \& Laub, J. H. (1993). Crime in the making: Pathways and turning points through life. Cambridge, MA, US: Harvard University Press. Retrieved from www.csa.com

Schmidt, F., Campbell, M. A., \& Houlding, C. (2011). Comparative analyses of the YLS/CMI, SAVRY, and PCL: YV in adolescent offenders: A 10-year follow-up into adulthood. Youth Violence and Juvenile Justice, 9, 23-42. doi:10.1177/1541204010371793

Schwalbe, C. S. (2007). Risk assessment for juvenile justice: A meta-analysis. Law and Human Behavior, 31, 449-462. doi:10.1007/s10979-006-9071-7

Seagrave, D., \& Grisso, T. (2002). Adolescent development and the measurement of juvenile psychopathy. Law and Human Behavior, 26, 219-239. doi:10.1023/A:1014696110850 
Serin, R. C., \& Lloyd, C. D. (2009). Examining the process of offender change: The transition to crime desistance. Psychology, Crime \& Law, 15, 347-364. doi:10.1080/10683160802261078

Sevecke, K., Kosson, D. S., \& Krischer, M. K. (2009). The relationship between attention deficit hyperactivity disorder, conduct disorder, and psychopathy in adolescent male and female detainees. Behavioral Sciences \& the Law, 27, 577-598. doi:10.1002/bs1.870

Silverthorn, P., \& Frick, P.J. (1999). Developmental issues in the clinical treatment of children. Boston: Allyn \& Bacon.

Singh, J. P., Grann, M., \& Fazel, S. (2011). A comparative study of violence risk assessment tools: A systematic review and metaregression analysis of 68 studies involving 25,980 participants. Clinical Psychology Review, 31, 499-513. doi:10.1016/j.cpr.2010.11.009

Skeem, J. L., \& Cauffman, E. (2003). Views of the downward extension: Comparing the youth version of the psychopathy checklist with the youth psychopathic traits inventory. Behavioral Sciences \& the Law, 21, 737-770. doi:10.1002/bs1.563

Skeem, J. L., Edens, J. F., Camp, J., \& Colwell, L. H. (2004). Are there ethnic differences in levels of psychopathy? A meta-analysis. Law and Human Behavior, 28, 505-527. doi:10.1023/B:LAHU.0000046431.93095.d8

Skeem, J. L., Monahan, J., \& Mulvey, E. P. (2002). Psychopathy, treatment involvement, and subsequent violence among civil psychiatric patients. Law and Human Behavior, 26, 577603. doi:10.1023/A:1020993916404 
Skeem, J. L., \& Mulvey, E. P. (2001). Psychopathy and community violence among civil psychiatric patients: Results from the MacArthur violence risk assessment study. Journal of Consulting and Clinical Psychology, 69, 358-374. doi:10.1037/0022-006X.69.3.358

Skilling, T. A., Quinsey, V. L., \& Craig, W. M. (2001). Evidence of a taxon underlying serious antisocial behavior in boys. Criminal Justice and Behavior. Special Issue: Psychopathy and Risk Assessment, 28, 450-470. doi:10.1177/009385480102800404

Smith, A. M., Gacono, C. B., \& Kaufman, L. (1997). A Rorschach comparison of psychopathic and nonpsychopathic conduct disordered adolescents. Journal of Clinical Psychology, 53, 289-300. doi:10.1002/(SICI)1097-4679(199706)53:4<289::AID-JCLP1>3.0.CO;2-L

Sorbello, L., Eccleston, L., Ward, T., \& Jones, R. (2002). Treatment needs of female offenders: A review. Australian Psychologist, 37, 198-205. doi:10.1080/00050060210001706876

Stattin, H., \& Magnusson, D. (1995). Onset of official delinquency: Its co-occurrence in time with educational, behavioural, and interpersonal problems. British Journal of Criminology, 35, 417-449. Retrieved from www.csa.com

Stockdale, K.C., Olver, M.E., \& Wong, S.C.P. (2010). The Psychopathy Checklist: Youth Version and adolescent and adult recidivism: Considerations with respect to gender, ethnicity, and age. Psychological Assessment, 22, 768-781, doi: 10.1037/a0020044

Stouthamer-Loeber, M., Loeber, R., Farrington, D. P., \& Zhang, Q. (1993). The double edge of protective and risk factors for delinquency: Interrelations and developmental patterns. Development and Psychopathology. Special Issue: Milestones in the Development of Resilience, 5, 683-701. doi:10.1017/S0954579400006234 
Stouthamer-Loeber, M., Wei, E., Loeber, R., \& Masten, A. S. (2004). Desistance from persistent serious delinquency in the transition to adulthood. Development and Psychopathology, 16, 897-918. doi:10.1017/S0954579404040064

Sullivan, E. A., Abramowitz, C. S., Lopez, M., \& Kosson, D. S. (2006). Reliability and construct validity of the psychopathy checklist-revised for Latino, European American, and African American male inmates. Psychological Assessment, 18, 382-392. doi:10.1037/10403590.18 .4 .382

Swailes, S., \& MacIntyre-Bhatty, T. (2002). The "Belbin" team role inventory: Reinterpreting reliability estimates. Journal of Managerial Psychology, 17, 529-536. doi: $10.1108 / 02683940210439432$

Vincent, G. M. (2006). Psychopathy and violence risk assessment in youth. Child and Adolescent Psychiatric Clinics of North America, 15, 407-428. doi:10.1016/j.chc.2005.12.001

Vincent, G. M., Chapman, J., \& Cook, N. E. (2011). Risk-needs assessment in juvenile justice: Predictive validity of the SAVRY, racial differences, and the contribution of needs factors. Criminal Justice and Behavior, 38, 42-62. doi:10.1177/0093854810386000

Vincent, G. M., \& Hart, S. D. (2002). Psychopathy in childhood and adolescence: Implications for the assessment and management of multi-problem youths. In R. R. Corrado, R. Roesch, S. D. Hart \& J. K. Gierowski (Eds.), Multi-problem violent youth: A foundation for comparative research on needs, interventions and outcomes (pp. 150-163).

Amsterdam, Netherlands: IOS Press. Retrieved from www.csa.com 
Vincent, G. M., Odgers, C. L., McCormick, A. V., \& Corrado, R. R. (2008). The PCL: YV and recidivism in male and female juveniles: A follow-up into young adulthood. International Journal of Law and Psychiatry. Special Issue: Psychopathic Traits and Risk Assessment in Children and Adolescents, 31, 287-296. doi:10.1016/j.ijlp.2008.04.012

Vincent, G. M., Vitacco, M. J., Grisso, T., \& Corrado, R. R. (2003). Subtypes of adolescent offenders: Affective traits and antisocial behavior patterns. Behavioral Sciences \& the Law, 21, 695-712. doi:10.1002/bsl.556

Walsh, Z., \& Kosson, D. S. (2007). Psychopathy and violent crime: A prospective study of the influence of socioeconomic status and ethnicity. Law and Human Behavior, 31, 209-229. doi:10.1007/s10979-006-9057-5

Ward, T., \& Brown, M. (2004). The good lives model and conceptual issues in offender rehabilitation. Psychology, Crime \& Law, 10, 243-257. doi: $10.1080 / 10683160410001662744$

Weiler, B. L., \& Widom, C. S. (1996). Psychopathy and violent behaviour in abused and neglected young adults. Criminal Behaviour and Mental Health, 6, 253-271. doi:10.1002/cbm.99

Welsh, J. L., Schmidt, F., McKinnon, L., Chattha, H. K., \& Meyers, J. R. (2008). A comparative study of adolescent risk assessment instruments: Predictive and incremental validity. Assessment, 15, 104-115. doi:10.1177/1073191107307966 
Wileman, B., Gullone, E., \& Moss, S. (2007). The juvenile persistent offender, primary group deficiency and persistent offending into adulthood: A prospective study (1980--2002). Psychiatry, Psychology and Law, 14, 66-77. doi:10.1375/pplt.14.1.66

Williamson, S.E. (1991). Cohesion and coherence in the speech of psychopathic criminals. Doctoral dissertation, University of British Columbia, Vancouver, Canada.

Williamson, S., Hare, R. D., \& Wong, S. (1987). Violence: Criminal psychopaths and their victims. Canadian Journal of Behavioural Science. Special Issue: Forensic Psychology, 19, 454-462. doi:10.1037/h0080003

Wolfgang, M. E., Thornberry, T. P., \& Figlio, R. M. (1987). From boy to man, from delinquency to crime. Chicago, IL, US: University of Chicago Press. Retrieved from www.csa.com

Woodworth, M., \& Porter, S. (2002). In cold blood: Characteristics of criminal homicides as a function of psychopathy. Journal of Abnormal Psychology, 111, 436-445. doi:10.1037/0021-843X.111.3.436

Zinger, I., \& Forth, A. E. (1998). Psychopathy and Canadian criminal proceedings: The potential for human rights abuses. Canadian Journal of Criminology, 40, 237-276. Retrieved from www.csa.com 
Appendix A

PCL:YV Item Descriptions

Ratings: $0=$ no; $1=$ maybe $2=$ yes; $x=$ omit

\begin{tabular}{|c|c|c|c|c|c|c|c|}
\hline & Factor 1 & Factor 2 & Factor 3 & Factor 4 & $\begin{array}{l}\text { Total } \\
\text { Score }\end{array}$ & & Item: \\
\hline 1 & & & & & & 1 & impression management \\
\hline 2 & & & & & & 2 & grandiose sense of self worth \\
\hline 3 & & & & & & 3 & stimulation seeking \\
\hline 4 & & & & & & 4 & pathological lying \\
\hline 5 & & & & & & 5 & manipulation for personal gain \\
\hline 6 & & & & & & 6 & lack of remorse \\
\hline 7 & & & & & & 7 & shallow affect \\
\hline 8 & & & & & & 8 & callous/lack of empathy \\
\hline 9 & & & & & & 9 & parasitic orientation \\
\hline 10 & & & & & & 10 & poor anger controls \\
\hline 11 & & & & & & 11 & impersonal sexual behaviour \\
\hline 12 & & & & & & 12 & early behavioural problems \\
\hline 13 & & & & & & 13 & lacks goals \\
\hline 14 & & & & & & 14 & impulsivity \\
\hline 15 & & & & & & 15 & irresponsibility \\
\hline 16 & & & & & & 16 & failure to accept responsibility \\
\hline 17 & & & & & & 17 & unstable interpersonal relationships \\
\hline 18 & & & & & & 18 & serious criminal behaviour \\
\hline 19 & & & & & & 19 & serious violations of cond. release \\
\hline 20 & & & & & & 20 & criminal versatility \\
\hline
\end{tabular}
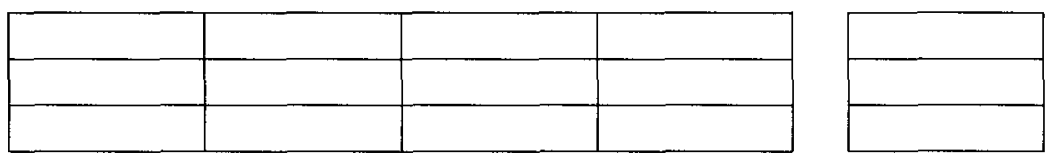

Raw sum

Number of missing items

Pro-rated score

PCL:YV $\square$ low (0 to 19.9) $\square$ medium (20 to 29.9) $\square$ High (30 to 40) 
Appendix B

\section{SAVRY Item Descriptions}

\section{Historical Risk}

Factors:

1 History of violence
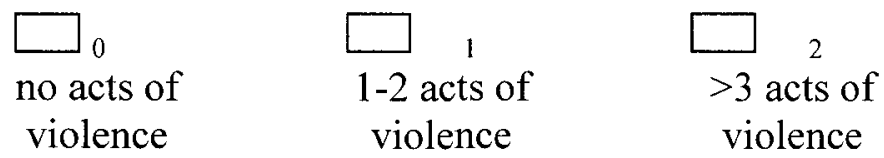

2 History of non-violent offending

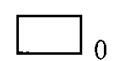

no prior nv offending
$<5$ prior nv offending

$>5$ prior acts $n v$ offending

3 Early initiation of violence

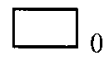

no violent acts

before 14 violence $b / w$ 11-13 yrs violence before age 11

\section{Past}

supervision/intervention failures
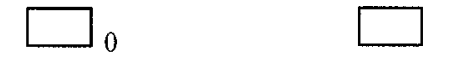

$$
1
$$

2

complied with

all court orders

failed to comply $>3$ times
5 History of self-harm or

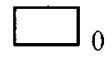
suicide attempts no history

\section{history with no clear suicide} intent
6 Exposure to violence in the home

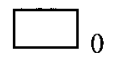

has not witnessed violence

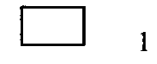

has witnessed phy. agg or 1 act serious viol.
2

history of self harm or suicide 
7 Childhood history of maltreatment

$\begin{array}{ccc}\square 0 & \square{ }_{1} & \square \\ \begin{array}{c}\square_{0} \\ \text { no physical } \\ \text { abuse or } \\ \text { neglect }\end{array} & \begin{array}{c}\text { infrequent or } \\ \text { less serious } \\ \text { abuse/neglect }\end{array} & \begin{array}{c}\text { chronic or } \\ \text { severe } \\ \text { abuse/neglect }\end{array}\end{array}$

8 Parental/caregiver criminality

9 Early caregiver disruption

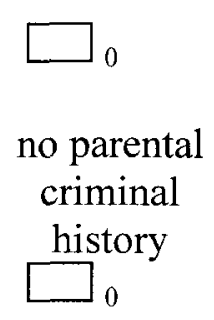

continuity of care during childhood

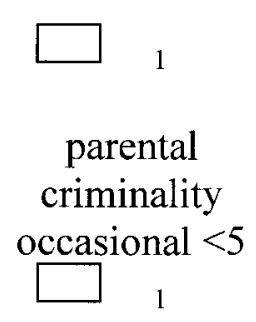

some discontinuity of care

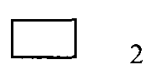

frequent $>5$ parental criminality 2

some discontinuity of care

10 Poor school achievement
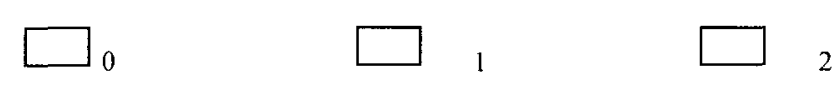

$$
\begin{gathered}
\text { no significant } \\
\text { difficulties }
\end{gathered}
$$

significant
difficulties

\section{Social/Contextual}

Risk Factors:
11 Peer delinquency
$\square 0$ Doesn't associate with delinquent peers
some difficulties


Persistence and Desistence of Offending 175

ability

coping

coping ability

14 Poor parental management

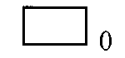

$$
\begin{aligned}
& \text { Consistent and } \\
& \text { appropriate } \\
& \text { parental } \\
& \text { management }
\end{aligned}
$$

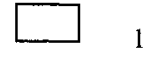

Somewhat inconsistent parental management

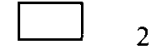

Extremely inconsistent or overly strict/permissive parental management
15 Lack of personal/social

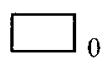
support

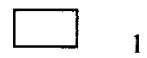

1

Inconsistent or
unreliable
emotional
support and

Few or no sources of emotional support and guidance 
16 Community disorganization

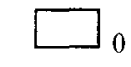

Low rates of crime, poverty, violence in community

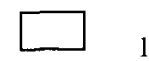

Some crime, poverty, violence problems in community

\section{Individual/Clinical}

Risk Factors:

17 Negative attitudes

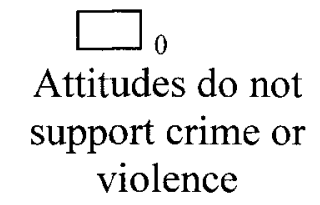
$\square \quad 1$
Some attitudes supportive of crime or violence

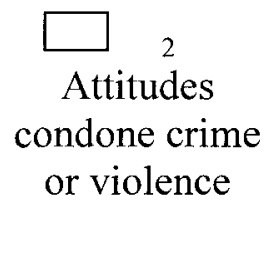

18 Risk taking/impulsivity

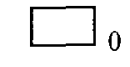

Exhibits no problems with risk taking / impulsivity
Exhibits minor risk taking / impulsivity

Significant crime, poverty, violence in community

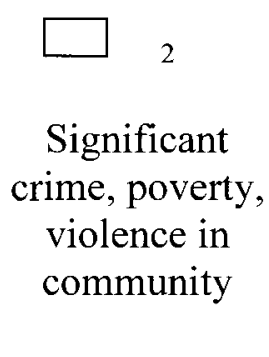




$\begin{array}{ccc}\text { Age appropriate } & \text { Moderate } & \text { Significant } \\ \text { capacity for } & \text { impairment in } & \text { impairment in } \\ \text { remorse / } & \text { capacity for } & \text { capacity for } \\ \text { empathy } & \text { remorse / } & \text { remorse / } \\ & \text { empathy } & \text { empathy }\end{array}$

22 Attention

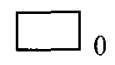

deficit/hyperactivity
No difficulties

$\mathrm{w} /$ restlessness, hyperactivity, or concentration

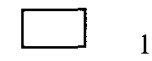

Moderate difficulties $\mathrm{w} /$ restlessness, hyperactivity, or concentration

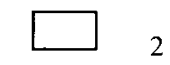

Serious difficulties with restlessness, hyperactivity

or concentration

23 Poor compliance

Positive attitude
toward
intervention,
authority

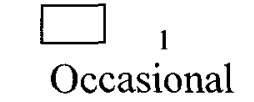
negative attitude toward intervention, treatment

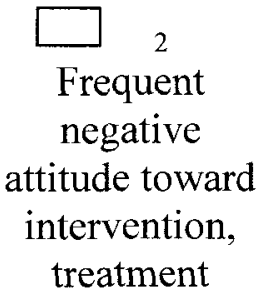

\section{Low} interest/commitment to school

$\begin{array}{cc}\square & \square \\ \begin{array}{c}\text { Average interest } \\ \text { / commitment to } \\ \text { school }\end{array} & \begin{array}{c}\text { Low interest / } \\ \text { commitment but } \\ \text { presently } \\ \text { attends and } \\ \text { completes } \\ \text { school work }\end{array}\end{array}$

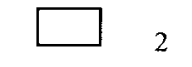

Low interest / commitment often truant, late, does not complete school work

\section{Protective Factors:}

P1 Prosocial involvement

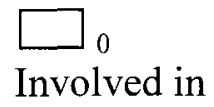
Little/no 


$\begin{array}{cc}\text { prosocial } & \text { involvement in } \\ \text { activities/peer } & \text { prosocial } \\ \text { groups } & \text { activities/peer } \\ & \text { groups }\end{array}$

P2 Strong social support

$\square 0$

Strong social supports $\square \quad 1$

No strong social supports

P3 Strong attachments and bonds<smiles>[CH]1CCC1</smiles>

Strong attachment/bond with $>1$ prosocial adult

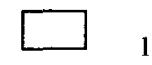

$$
\begin{gathered}
\text { No } \\
\text { attachment/bond } \\
\text { with }>1 \\
\text { prosocial adult }
\end{gathered}
$$

P4 Positive attitude toward intervention and authority
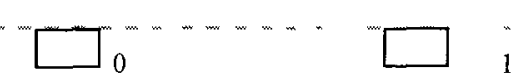

$$
\begin{array}{cc}
\text { Positive attitude } & \text { Not positive } \\
\text { toward } & \text { attitude toward } \\
\text { remediation / } & \text { remediation / } \\
\text { authority } & \text { authority }
\end{array}
$$

P5 Strong commitment to school<smiles>C1CCC1</smiles>

Exhibits high levels of interest, involvement, motivation
Des not exhibit high levels of interest, involvement, motivation

P6 Resilient personality traits

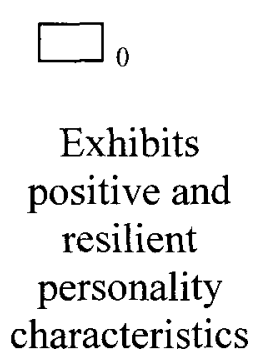


Appendix C

Conduct Disorder Items

Conduct Disorder Symptoms

1. Often bullies, threatens or intimidates others

2. Often initiates physical fights

3. has used a weapon that can cause serious physical harm to others

4. has stolen while confronting a victim

5. Has been physically cruel to people

6. Has been physically cruel to animals

7. Has forced someone into sexual activity

8. Often lies to obtain goods or favours or to avoid obligations

9. Often stays out at night despite parental prohibitions, beginning before 13 years

10. Has stolen items of non-trivial value without confronting a victim

11. Has deliberately engaged in fire setting with the intention of causing serious damage

12. Has deliberately destroyed others' property (other than by fire setting)

13. Has run away from home overnight at least twice

14. Is often truant from school, beginning before age 13

15. Has broken into someone else's house, building or car 
Appendix D

\section{Coding Guide for Offences}

ID:

FPS \#:

\section{Official Criminal History Record:}

Enter the number of offences (i.e. convictions) for each individual crime. If there are none, enter 0 . This section is to be coded exclusively from the official records contained in CPIC files, and includes only charges and convictions prior to the index offence.

\section{Non-Violent Offences:}

1.

2.

3.

4.

5.

6.

7.

8.

9.

10. 111

11.
Theft, break \& enter, possession of housebreaking tools, possession of stolen property, theft of communications, disguise with intent, forcible entry, unlawfully in a dwelling house

drug offences (i.e. possession and trafficking)

Criminal negligence (includes serious driving offences such as impaired or dangerous driving, failure to stop at the scene of an accident, hit-and-run)

fraud, forgery false pretences, impersonation, uttering, possession of stolen credit card.

escape, unlawfully at large, prison breaches

obstruction of justice, perjury, contempt of court, resist arrest, give contrary evidence

fail to appear in court, fail to comply with recognizance, fail to comply with probation order, breach of probation, breach of recognizance.

miscellaneous offence: vandalism, causing a disturbance, mischief, wilful damage, trespassing, conspiracy to commit a non-violent offence, vagrancy, prostitution, minor driving offences (i.e. driving with suspended licence), public intoxication.

total of above offences

date of first non-violent conviction $(\mathrm{mm} / \mathrm{dd} / \mathrm{yy})$

number of non-violent conviction (sentence) dates 
12. r__ robbery, armed robbery, robbery with violence, extortion

13.___ arson, fire setting

14.___ uttering threats, participation in riots, intimidation

15. ___ assault, assault causing bodily harm, wounding with intent, malicious wounding, aggravated assault, aggravated assault causing bodily harm

16. possession of a weapon, possession of explosives, pointing a firearm

17. ___ kidnapping, abduction, forcible confinement, forcible seizure

18. ___ manslaughter

19. __ attempted murder, attempted manslaughter, conspiracy to commit murder

20. $\quad$ second degree murder

21. ___ first degree murder

22. ___ total of above offences

23. _ _ date of first violent conviction $(\mathrm{mm} / \mathrm{dd} / \mathrm{yy})$

24. number of violent conviction (sentence) dates

\section{Sexual Offences:}

25. ___ sexual assault, sexual assault causing bodily harm

26. ___ aggravated sexual assault, sexual assault with a weapon

27.___ sexual interference

28. $\quad$ total of above offences

29. I _ _ date of first sexual conviction $(\mathrm{mm} / \mathrm{dd} / \mathrm{yy})$

30. number of sexual conviction (sentence) dates 


\section{Index Offences}

\section{Official Criminal Record:}

Enter the number of offences (i.e. convictions) for each individual crime. If there are none, enter 0 . This section is to be coded exclusively from the official records contained in CPIC files, and includes only charges and convictions related to the index offence

\section{Non-Violent Offences:}

1.

Theft, break \& enter, possession of housebreaking tools, possession of stolen property, theft of communications, disguise with intent, forcible entry, unlawfully in a dwelling house

2.

drug offences (i.e. possession and trafficking)

3.

Criminal negligence (includes serious driving offences such as impaired or dangerous driving, failure to stop at the scene of an accident, hit-and-run)

4.

fraud, forgery false pretences, impersonation, uttering, possession of stolen credit card.

5.

6.

escape, unlawfully at large, prison breaches

obstruction of justice, perjury, contempt of court, resist arrest, give contrary evidence

7.

fail to appear in court, fail to comply with recognizance, fail to comply with probation order, breach of probation, breach of recognizance.

8.

miscellaneous offence: vandalism, causing a disturbance, mischief, wilful damage, trespassing, conspiracy to commit a non-violent offence, vagrancy, prostitution, minor driving offences (i.e. driving with suspended licence), public intoxication.

9. $\quad$ total of above offences

10. $1 / 1$ date of index non-violent conviction $(\mathrm{mm} / \mathrm{dd} / \mathrm{yy})$

11. __ _ date of release for index conviction/remand $(\mathrm{mm} / \mathrm{dd} / \mathrm{yy})$ 


\section{Violent Offences:}

12. robbery, armed robbery, robbery with violence, extortion

13. arson, fire setting

14. uttering threats, participation in riots, intimidation

15. assault, assault causing bodily harm, wounding with intent, malicious wounding, aggravated assault, aggravated assault causing bodily harm

16. possession of a weapon, possession of explosives, pointing a firearm

17. kidnapping, abduction, forcible confinement, forcible seizure

18. manslaughter

19. attempted murder, attempted manslaughter, conspiracy to commit murder

20. second degree murder

21. first degree murder

22. total of above offences

23. $1 \ldots$ date of index violent conviction $(\mathrm{mm} / \mathrm{dd} / \mathrm{yy})$

24.

\section{Sexual Offences:}

25. sexual assault, sexual assault causing bodily harm

26. aggravated sexual assault, sexual assault with a weapon

27. sexual interference

28. total of above offences

29. I__ _ date of index sexual conviction $(\mathrm{mm} / \mathrm{dd} / \mathrm{yy})$

30. _ _ _ _ date of release for index conviction $/ \mathrm{remand}(\mathrm{mm} / \mathrm{dd} / \mathrm{yy})$ 


\section{Official Recidivism Offences}

\section{Official Criminal Record:}

Enter the number of offences (i.e. convictions) for each individual crime. If there are none, enter 0 . This section is to be coded exclusively from the official records contained in CPIC files, and includes only charges and convictions subsequent to release for index offence and prior to young offender's $18^{\text {th }}$ birthday (if applicable)

\section{Non-Violent Offences:}

1.

2.

3.

4.

5.

6.

7.

8.

9.

10.11

11. 11
Theft, break \& enter, possession of housebreaking tools, possession of stolen property, theft of communications, disguise with intent, forcible entry, unlawfully in a dwelling house

drug offences (i.e. possession and trafficking)

Criminal negligence (includes serious driving offences such as impaired or dangerous driving, failure to stop at the scene of an accident, hit-and-run)

fraud, forgery false pretences, impersonation, uttering, possession of stolen credit card.

escape, unlawfully at large, prison breaches

obstruction of justice, perjury, contempt of court, resist arrest, give contrary evidence

fail to appear in court, fail to comply with recognizance, fail to comply with probation order, breach of probation, breach of recognizance.

miscellaneous offence: vandalism, causing a disturbance, mischief, wilful damage, trespassing, conspiracy to commit a non-violent offence, vagrancy, prostitution, minor driving offences (i.e. driving with suspended licence), public intoxication.

total of above offences

date of first non-violent conviction $(\mathrm{mm} / \mathrm{dd} / \mathrm{yy})$ prior to $18^{\text {th }}$ birthday

date of last non-violent conviction ( $\mathrm{mm} / \mathrm{dd} / \mathrm{yy})$ prior to $18^{\text {th }}$ birthday 
12. robbery, armed robbery, robbery with violence, extortion

13. arson, fire setting

14. uttering threats, participation in riots, intimidation

15. assault, assault causing bodily harm, wounding with intent, malicious wounding, aggravated assault, aggravated assault causing bodily harm

16. possession of a weapon, possession of explosives, pointing a firearm

17. kidnapping, abduction, forcible confinement, forcible seizure

18. manslaughter

19. attempted murder, attempted manslaughter, conspiracy to commit murder

20. second degree murder

21. first degree murder

22. total of above offences

23. _ _ _ date of first violent conviction $(\mathrm{mm} / \mathrm{dd} / \mathrm{yy})$ prior to $18^{\text {th }}$ birthday

24. _ _ date of last violent conviction $(\mathrm{mm} / \mathrm{dd} / \mathrm{yy})$ prior to $18^{\text {th }}$ birthday

\section{Sexual Offences:}

25. sexual assault, sexual assault causing bodily harm

26. aggravated sexual assault, sexual assault with a weapon

27. sexual interference

28. total of above offences

29. _ _ _ date of first sexual conviction $(\mathrm{mm} / \mathrm{dd} / \mathrm{yy})$ prior to $18^{\text {th }}$ birthday 30. I _ _ date of last sexual conviction $(\mathrm{mm} / \mathrm{dd} / \mathrm{yy})$ prior to $18^{\text {th }}$ birthday 


\section{Appendix E}

\section{Ethics Approval Certificate}

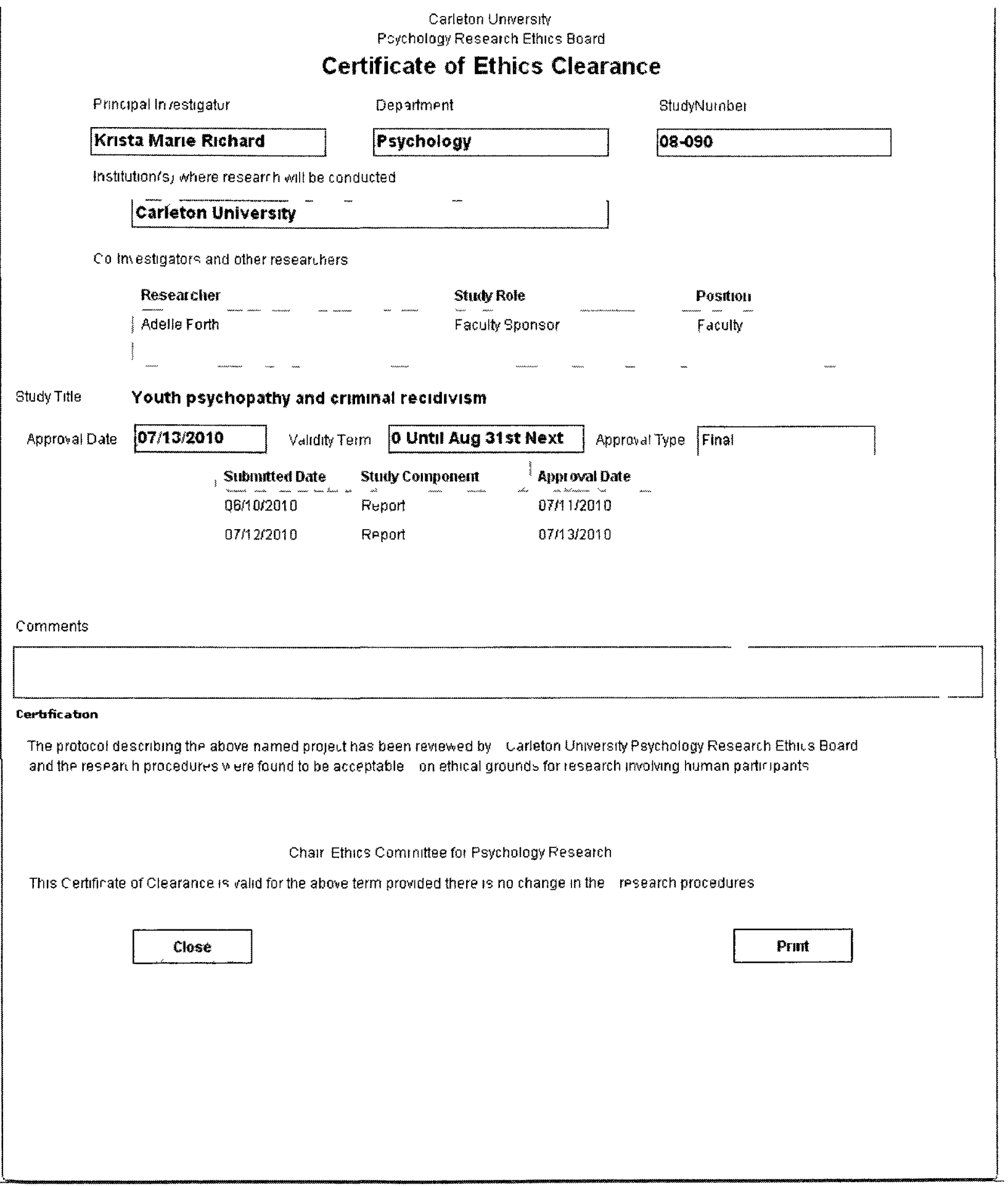




\section{Appendix F}

Comparisons of the Three Samples

\section{Sample Comparisons}

Age and Education. Table 37 presents the breakdown of age for each of the three samples. A significant difference was found on the mean scores across the three samples, $F(2$, $232)=33.52, p<.001$. Post-hoc comparisons revealed that sample 1 was significantly younger at time of original assessment than were samples 2 and 3. Education levels were also compared for differences across each of the samples. A significant main effect was found for education, $F$ $(2,195)=4.82, p<.01$ indicating that sample 1 completed less education grade levels than the other two samples.

Ethnicity. When ethnicity was compared across the three samples, significant differences emerged, $\chi^{2}(230)=43.5, p<.001$ (see Table 38). Sample 1 had more Aboriginal offenders than the other two samples and Sample 3 contained less Caucasian offenders than the other two groups. Sample 3 had 10\% of the offenders identifying as being Black, whereas Sample 1 had none and Sample 2 only had one offender identifying himself as Black.

Number of Previous Convictions. In examining the number of prior convictions for non-violent, violent and sexual offences committed, several differences were found between the groups. On the number of prior non-violent convictions, a significant main effect was found, $F$ $(2,232)=4.18, p<.02$. Upon examining post-hoc comparisons, it was found that Sample $3 \mathrm{had}$ committed more non-violent offences than Sample 1. No other differences were significant for non-violent offending. For the number of prior violent convictions, again, a significant main effect was found, $F(2,232)=15.9, p<.001$. Sample 3 was found to have committed more violent offences than both Sample 1 and Sample 2. No significant differences were found 
between the three samples on the number of prior sexual convictions, $F(2,232)=0.08, p=.92$ (see Table 39).

Number of Index Offences. When the number of non-violent offences committed at the time of assessment was examined, no differences were found between each of the three samples, $F(2,232)=1.86, p=.16$, indicating that all three groups committed similar number of nonviolent offences. Similar findings were noted for violent offences, $F(2,232)=2.94, p=.054$ and for sexual offences, $F(2,232)=0.81, p=.44$ (see Table 39).

Psychopathy. The overall sample had an average total PCL:YV score of $24.8(S D=8.0)$, however when comparisons were examined across the three samples, differences were found, $F$ $(2,232)=15.5, p<.001$. Sample 3 was found to have significantly lower PCL:YV total scores than Samples 1 and 2. Sample 2 had the highest total score out of all three samples, but did not differ significantly from Sample 1. In examining each of the factor scores, Factor 1 was found to be different between Sample 2 and Sample 3, $F(2,232)=3.3, p<.04$, with Sample 3 having a significantly lower factor score than sample 2 (see Table 37).

Risk for Violence. A significant main effect was found for SAVRY risk across the three different samples, $F(2,231)=8.71, p<.001$. The sample from 1994 (Sample 2) was found to have significantly lower scores than what was found for the other two Samples. Furthermore, Sample 2 was found to have lower Historical scores, $F(2,231)=7.78, p<.001$, lower Social scores, $F(2,231)=9.67, p<.001$ and Clinical ratings $F(2,231)=5.26, p<.006$ when compared with the other two samples. Sample 1 was found to have significantly less protective scores than the other two samples, $F(2,231)=19.39, p<.001$ (see Table 37).

Conduct Disorder. When the number of conduct disorder symptoms was examined, a significant main effect was noted, $F(2,232)=20.19, p<.001$. When post-hoc comparisons 
were examined, Sample 1 was found to have significantly more CD symptoms than what was found within Samples 2 and 3 (see Table 37).

Table 37

Demographic and Outcome Measure Comparison Across the three Samples

\begin{tabular}{|c|c|c|c|c|}
\hline Variable & $\mathbf{M}$ & SD & Min. & Max. \\
\hline \multicolumn{5}{|l|}{ Age } \\
\hline Sample 1 & 16.3 & 1.1 & 13 & 20 \\
\hline Sample 2 & 17.5 & 0.9 & 15 & 20 \\
\hline Sample 3 & 17.2 & 0.9 & 16 & 20 \\
\hline \multicolumn{5}{|l|}{ Education } \\
\hline Sample 1 & 8.5 & 1.4 & 5 & 11 \\
\hline Sample 2 & 9.1 & 1.5 & 4 & 12 \\
\hline Sample 3 & 9.2 & 1.2 & 6 & 11 \\
\hline \multicolumn{5}{|c|}{ PCL:YV total } \\
\hline Sample 1 & 25.4 & 8.1 & 9 & 39 \\
\hline Sample 2 & 26.8 & 5.9 & 8 & 36.7 \\
\hline Sample 3 & 20.1 & 9.1 & 5 & 34 \\
\hline \multicolumn{5}{|c|}{ PCL:YV Factor 1} \\
\hline Sample 1 & 3.8 & 2.5 & 0 & 8 \\
\hline Sample 2 & 4.2 & 2.3 & 0 & 8 \\
\hline Sample 3 & 3.2 & 2.2 & 0 & 8 \\
\hline \multicolumn{5}{|c|}{ PCL:YV Factor 2} \\
\hline Sample 1 & 5.3 & 2.2 & 1 & 8 \\
\hline Sample 2 & 5.3 & 2.0 & 0 & 8 \\
\hline Sample 3 & 3.7 & 2.7 & 0 & 8 \\
\hline \multicolumn{5}{|c|}{ PCL:YV Factor 3} \\
\hline Sample 1 & 6.8 & 2.3 & 0 & 10 \\
\hline Sample 2 & 7.1 & 1.8 & 2 & 10 \\
\hline Sample 3 & 5.6 & 2.3 & 1 & 10 \\
\hline \multicolumn{5}{|c|}{ PCL:YV Factor 4} \\
\hline Sample 1 & 7.5 & 2.1 & 2 & 10 \\
\hline Sample 2 & 7.6 & 2.1 & 2 & 10 \\
\hline Sample 3 & 5.9 & 2.8 & 1 & 10 \\
\hline
\end{tabular}




$\begin{array}{lllll}\text { Variable } & \text { M } & \text { SD } & \text { Min. } & \text { Max }\end{array}$

\section{SAVRY risk}

Sample 1

Sample 2

Sample 3
26.2

22

25.9
7.5

7.0

8.1

3.6

3.5

3.6

9.6

Sample 3

\section{SAVRY Social}

Sample 1

Sample 2

Sample 3

\section{SAVRY Clinical}

\section{Sample 1}

Sample 2

Sample 3

SAVRY Protective

\section{Sample 1}

Sample 2

Sample 3

\section{CD \# symptoms}

Sample 1

Sample 2

Sample 3

$\begin{array}{ll}5.5 & 2.4 \\ 4.4 & 1.8 \\ 5.9 & 2.4\end{array}$

9.8

8.8

10.6

3.2

3.2

3.6

0.9

1.1

1.7

1.9

9.6

7.2

1.8

2.9

7.6
10

43

37

43

6
10

\section{3

3

(1)

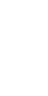

$\begin{array}{ll}1 & 17 \\ 2 & 18\end{array}$

Note: Sample 1 represents participants who were originally assessed in 1986, sample 2 represents participants originally assessed in 1994, and sample 3 represents participants assessed in 2004; $M$ is the mean score, $S D$ is the standard deviation, Min. is the minimum score obtained on the scale and Max. is the maximum score obtained on the scale. 
Table 38

Ethnic Comparisons Across the Three Samples

\begin{tabular}{lccc}
\hline Ethnicity & $\begin{array}{c}\text { Sample } 1 \\
n=75\end{array}$ & $\begin{array}{c}\text { Sample } 2 \\
n=103\end{array}$ & $\begin{array}{c}\text { Sample } 3 \\
n=57\end{array}$ \\
\hline Caucasian & $57(76.0)$ & $80(81.6)$ & $38(66.7)$ \\
Black & $0(0)$ & $1(1.0)$ & $10(17.4)$ \\
Aboriginal & $18(24.0)$ & $9(9.2)$ & $6(10.5)$ \\
Hispanic & $0(0)$ & $2(2.0)$ & $1(1.8)$ \\
Asian & $0(0)$ & $4(4.1)$ & $0(0)$ \\
Other & $0(0)$ & $2(2.0)$ & $2(3.5)$ \\
Missing & $0(0)$ & &
\end{tabular}

Note: percentage of each sample is given in parentheses.

Table 39

Prior and Index Offence Rates Across the Three Samples

Prior Offences

\begin{tabular}{lccc} 
Variable & $\begin{array}{c}\text { Non-Violent } \\
\%\end{array}$ & $\begin{array}{c}\text { Violent } \\
\%\end{array}$ & $\begin{array}{c}\text { Sexual } \\
\%\end{array}$ \\
\hline Sample 1 & 70.6 & 16.0 & 2.7 \\
Sample 2 & 83.5 & 45.6 & 1.9 \\
Sample 3 & 84.2 & 63.2 & 1.8 \\
Total & 79.6 & 40.4 & 2.1 \\
& & & \\
& & Index Offences & \\
& & & Sexual \\
& Non-Violent & Violent & 8.0 \\
Sample 1 & $\%$ & $\%$ & 8.7 \\
Sample 2 & & & 3.5 \\
Sample 3 & 82.7 & 22.6 & 7.2 \\
Total & 81.6 & 41.7 & \\
& 82.4 & 54.4 & \\
\hline
\end{tabular}


To discern whether the samples could be merged for outcome prediction analyses, comparisons were examined on whether significant differences emerged on AUCs across each of the three cohorts (see Tables 40, 41, and 42). As indicated in these tables, few differences were noted with none of the samples having non-overlapping $95 \%$ confidence intervals.

Table 40

Comparisons Across Samples on Predictive Accuracy of Risk Measures for General Recidivism

\begin{tabular}{|c|c|c|c|c|c|c|}
\hline & \multicolumn{2}{|c|}{ Sample 1} & \multicolumn{2}{|c|}{ Sample 2} & \multicolumn{2}{|c|}{ Sample 3} \\
\hline & AUC & $95 \% \mathrm{CI}$ & AUC & $95 \% \mathrm{CI}$ & AUC & $95 \% \mathrm{CI}$ \\
\hline & \multicolumn{6}{|c|}{ One-Year Follow-up } \\
\hline PCL:YV total & .61 & $.47, .74$ & .54 & $.43, .66$ & .58 & $.42, .73$ \\
\hline SAVRY risk & .58 & $.45, .72$ & .61 & $.50, .73$ & .52 & $.36, .67$ \\
\hline SAVRY Protective $\mathrm{a}^{\mathrm{a}}$ & .53 & $.39, .66$ & .64 & $.63, .75$ & .62 & $.47, .76$ \\
\hline \multirow[t]{2}{*}{ CD symptoms } & .64 & $.51, .77$ & .60 & $.49, .72$ & .53 & $.38, .69$ \\
\hline & \multicolumn{6}{|c|}{ Five-Year Follow-up } \\
\hline PCL:YV total & .61 & $.49, .72$ & .61 & $.49, .72$ & .60 & $.38, .82$ \\
\hline SAVRY risk & .57 & $.55, .76$ & .66 & $.55, .76$ & .59 & $.36, .82$ \\
\hline SAVRY Protective ${ }^{a}$ & .56 & $.40, .71$ & .71 & $.60, .81$ & .61 & $.40, .82$ \\
\hline \multirow[t]{2}{*}{ CD symptoms } & .58 & $.48, .71$ & .60 & $.48, .71$ & .58 & $.36, .81$ \\
\hline & \multicolumn{6}{|c|}{ Ten-Year Follow-up } \\
\hline PCL:YV total & .56 & $.39, .73$ & .59 & $.47, .70$ & -- & -- \\
\hline SAVRY risk & .54 & $.38, .71$ & .66 & $.55, .78$ & -- & -- \\
\hline SAVRY Protective ${ }^{a}$ & .55 & $.38, .72$ & .71 & $.60, .81$ & -- & -- \\
\hline CD symptoms & .52 & $.32, .72$ & .59 & $.47, .71$ & -- & -- \\
\hline
\end{tabular}


Table 41

Comparisons Across Samples on Predictive Accuracy of Risk Measures for Non-Violent Recidivism

Sample 1

AUC $\quad 95 \% \mathrm{CI}$
Sample 2

AUC $\quad 95 \% \mathrm{CI}$
Sample 3

AUC $\quad 95 \% \mathrm{CI}$

PCL:YV total

SAVRY risk

SAVRY Protective ${ }^{a}$

CD symptoms

PCL:YV total

SAVRY risk

SAVRY Protective ${ }^{a}$

CD symptoms
.61

.57

.54

.60
.61

.58

.52

.64
$.47, .74$

$.33, .77$

$.39, .66$

$.38, .80$

One-Year Follow-up

$\begin{array}{llll}.54 & .42, .67 & .58 & .42, .73 \\ .59 & .48, .71 & .52 & .36, .67 \\ .63 & .51, .74 & .61 & .47, .76 \\ .59 & .47, .71 & .53 & .38, .69\end{array}$

Five-Year Follow-up

$\begin{array}{llll}.58 & .47, .68 & .56 & .36, .77 \\ .61 & .50, .73 & .55 & .33, .77 \\ .67 & .57, .78 & .58 & .38, .79 \\ .60 & .48, .71 & .59 & .38, .80\end{array}$

Ten-Year Follow-up

PCL:YV total

SAVRY risk

SAVRY Protective ${ }^{a}$

CD symptoms

$$
\begin{aligned}
& .45, .76 \\
& .42, .72 \\
& .39, .69 \\
& .43, .76
\end{aligned}
$$

$.39, .71$

$.38, .70$

$.37, .68$

$.39, .75$
.56

.60

.64

.59
$.44, .67$

$.48, .72$

$.53, .76$

$.47, .71$

Note: PCL:YV = Psychopathy Checklist: Youth Version, SAVRY = Structured Assessment of Violence Risk in Youth, $\mathrm{CD}=$ conduct disorder; $\mathrm{AUC}=$ area under the curve; $\mathrm{CI}=$ Confidence Interval, $\mathrm{a}=$ for the SAVRY protective score, the prediction was for non-offending. 
Table 42

Comparisons Across Samples on Predictive Accuracy of Risk Measures for Violent Recidivism

\begin{tabular}{|c|c|c|c|c|c|c|}
\hline & \multicolumn{2}{|c|}{ Sample 1} & \multicolumn{2}{|c|}{ Sample 2} & \multicolumn{2}{|c|}{ Sample 3} \\
\hline & AUC & $95 \% \mathrm{CI}$ & AUC & $95 \% \mathrm{CI}$ & $\mathrm{AUC}$ & $95 \% \mathrm{CI}$ \\
\hline & \multicolumn{6}{|c|}{ One-Year Follow-up } \\
\hline PCL:YV total & .66 & $.37, .95$ & .65 & $.51, .80$ & .83 & $.73, .93$ \\
\hline SAVRY risk & .60 & $.31, .89$ & .64 & $.50, .78$ & .62 & $.47, .78$ \\
\hline SAVRY Protective $^{\mathrm{a}}$ & .36 & $.04, .68$ & .61 & $.42, .79$ & .59 & $.42, .76$ \\
\hline \multirow[t]{2}{*}{ CD symptoms } & .75 & $.57, .93$ & .58 & $.39, .78$ & .74 & $.59, .88$ \\
\hline & \multicolumn{6}{|c|}{ Five-Year Follow-up } \\
\hline PCL:YV total & .60 & $.47, .74$ & .62 & $.51, .73$ & .67 & $.53, .81$ \\
\hline SAVRY risk & .55 & $.41, .69$ & .70 & $.60, .80$ & .61 & $.45, .76$ \\
\hline SAVRY Protective ${ }^{\mathrm{a}}$ & .52 & $.38, .66$ & .65 & $.55, .76$ & .61 & $.46, .76$ \\
\hline \multirow[t]{2}{*}{ CD symptoms } & .62 & $.48, .75$ & .62 & $.51, .73$ & .62 & $.46, .77$ \\
\hline & \multicolumn{6}{|c|}{ Ten-Year Follow-up } \\
\hline PCL:YV total & .51 & $.37, .64$ & .64 & $.53, .75$ & -- & -- \\
\hline SAVRY risk & .57 & $.44, .70$ & .74 & $.65, .84$ & -- & -- \\
\hline SAVRY Protective $^{\mathrm{a}}$ & .56 & $.42, .69$ & .67 & $.57, .77$ & -- & - \\
\hline CD symptoms & .54 & $.54, .68$ & .64 & $.53, .75$ & -- & -- \\
\hline
\end{tabular}

Note: PCL:YV = Psychopathy Checklist: Youth Version, SAVRY = Structured Assessment of Violence Risk in Youth, $\mathrm{CD}=$ conduct disorder; $\mathrm{AUC}=$ area under the curve; $\mathrm{CI}=$ Confidence Interval, $\mathrm{a}=$ for the SAVRY protective score, the prediction was for non-offending. 